\title{
Matrix factorisations for rational boundary conditions by defect fusion
}

\author{
Nicolas Behr ${ }^{a, b}$ and Stefan Fredenhagen ${ }^{c}$ \\ ${ }^{a}$ Department of Mathematics, Heriot-Watt University, \\ Riccarton, Edinburgh, EH14 4AS, U.K. \\ ${ }^{b}$ Maxwell Institute for Mathematical Sciences, \\ Edinburgh, U.K. \\ ${ }^{c}$ Max-Planck-Institut für Gravitationsphysik, Albert-Einstein-Institut, \\ D-14424 Golm, Germany \\ E-mail: N.Behr@hw.ac.uk, Stefan.Fredenhagen@aei.mpg.de
}

ABSTRACT: A large class of two-dimensional $\mathcal{N}=(2,2)$ superconformal field theories can be understood as IR fixed-points of Landau-Ginzburg models. In particular, there are rational conformal field theories that also have a Landau-Ginzburg description. To understand better the relation between the structures in the rational conformal field theory and in the Landau-Ginzburg theory, we investigate how rational B-type boundary conditions are realised as matrix factorisations in the $\mathrm{SU}(3) / \mathrm{U}(2)$ Grassmannian Kazama-Suzuki model. As a tool to generate the matrix factorisations we make use of a particular interface between the Kazama-Suzuki model and products of minimal models, whose fusion can be realised as a simple functor on ring modules. This allows us to formulate a proposal for all matrix factorisations corresponding to rational boundary conditions in the $\mathrm{SU}(3) / \mathrm{U}(2)$ model.

Keywords: D-branes, Conformal Field Models in String Theory, Tachyon Condensation, Topological Field Theories

ARXIV EPRINT: 1407.7254 


\section{Contents}

1 Introduction $\quad 2$

1.1 Plan of the paper and summary of results 3

2 Matrix factorisations and variable transformation interfaces 4

2.1 Matrix factorisations in Landau-Ginzburg models 4

2.2 Variable transformation interfaces 5

2.3 Kazama-Suzuki models 7

3 Boundaries and defects in Kazama-Suzuki models $\quad 8$

3.1 Bulk theory 8

3.2 Boundary conditions $\quad 9$

$\begin{array}{lll}3.3 & \text { Boundary renormalisation group flows } & 10\end{array}$

$\begin{array}{ll}3.4 & \text { Defects and fusion }\end{array}$

4 Matrix factorisations for rational boundary conditions 11

$\begin{array}{lll}4.1 & \text { Polynomial factorisations } & 12\end{array}$

$\begin{array}{lll}4.2 & \text { RR-charges } & 13\end{array}$

$\begin{array}{lll}4.3 & \text { Higher factorisations from cones } & 16\end{array}$

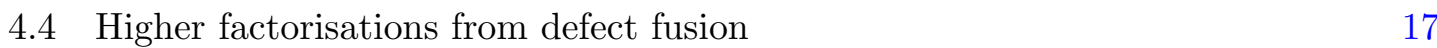

$\begin{array}{ll}\text { 4.5 Matrix factorisations for all rational boundary conditions } & 19\end{array}$

4.5.1 A closed formula for rational matrix factorisations $Q_{|0, \ell\rangle} \quad 20$

4.5.2 A closed formula for all rational matrix factorisations 23

$\begin{array}{lll}4.6 & \text { Effects of finite levels } & 27\end{array}$

5 Conclusion and outlook $\quad 32$

$\begin{array}{ll}\text { A Similarity transformations } & 33\end{array}$

B Multiple defect action on polynomial factorisations 34

$\begin{array}{ll}\text { C Deriving the alternative standard form } & 40\end{array}$

$\begin{array}{ll}\text { D Constraints at finite level } & 45\end{array}$

E A closed $2 \times 2$ form for $\mathcal{E}_{n(m)}$ and $\mathcal{J}_{n(m)}$ 


\section{Introduction}

$\mathcal{N}=(2,2)$ superconformal field theories play an important role as world-sheet descriptions of superstrings. There are various constructions and approaches known: the geometric construction as non-linear sigma model, rational coset constructions (Kazama-Suzuki models), and the realisation as infrared fixed-point of a supersymmetric Landau-Ginzburg model (see e.g. [1] for a review). Each approach has advantages and disadvantages, in the sense that there are certain quantities that are easy to compute, and others that are difficult. For example, in the rational construction one has good control over the correlation functions, and many quantities can be determined exactly, but on the other hand, it is hard to compute deformations of the theory, because the large rational symmetry is then broken. In contrast to that, in Landau-Ginzburg models deformations of the superpotential are easily described, but only few quantities can be computed exactly, namely those that are protected when one follows the renormalisation group flow to the infrared. It is therefore desirable to make contact between the different approaches to combine the advantages and to learn more about the different descriptions. One example of such a connection is given by gauged linear sigma models, which provide a relation between geometric models and certain Landau-Ginzburg orbifolds [2], and in this way one has obtained a good understanding of the moduli space of such theories.

We are interested here in the connection between rational theories and their LandauGinzburg realisation. It is known that there is a large class of supersymmetric coset models that have a Landau-Ginzburg description, a subclass of the Kazama-Suzuki models $[3,4]$. Within this class there are the Grassmannian Kazama-Suzuki models that have a description as cosets $\mathrm{SU}(n+1)_{k} / \mathrm{U}(n)$. The superpotentials of the corresponding LandauGinzburg theories have been identified in $[5,6]$, relying on the identification of the chiral ring of bulk fields.

In rational theories, one also has a distinguished family of rational boundary conditions and defects, and it is therefore natural to study those and to look for their counterparts on the Landau-Ginzburg side. This has been studied for (products of) minimal models and orbifolds thereof in [7-12]. In these models the rational algebras are (products of) super-Virasoro algebras, so that the algebraic structures are rather simple. A non-minimal situation has been explored in [13], where we identified matrix factorisations for some rational boundary conditions in the $\mathrm{SU}(3) / \mathrm{U}(2)$ Kazama-Suzuki model. The strategy there was to identify first some elementary factorisations, and then build others with the help of the cone construction as tachyon condensates of elementary ones. This approach, however, cannot be driven very far, because the cones in question quickly become very complicated.

In this work we want to continue to study the $\mathrm{SU}(3) / \mathrm{U}(2)$ model, but following a different approach. The idea is to generate new boundary conditions by fusing defects onto known boundary conditions. If we have identified the appropriate defects as matrix factorisations, we can use them to generate new matrix factorisations for boundary conditions from known ones by taking tensor products of matrix factorisations.

To identify matrix factorisations for defects, we make use of an interface between the $\mathrm{SU}(3) / \mathrm{U}(2)$ Kazama-Suzuki model and the product of two minimal models that we intro- 
duced in [14]. The fusion of this variable transformation interface to a matrix factorisation has a simple operator-like description: it can be implemented by a simple operation acting individually on each entry of the matrix factorisation.

Fusing this interface to a matrix factorisation in the minimal models results in a matrix factorisation for the Kazama-Suzuki model. This interface then allows us to identify a matrix factorisation for a particular rational topological defect in the Kazama-Suzuki model. Fusing this defect to the matrix factorisations identified in [13], we generate matrix factorisations for all rational boundary conditions.

\subsection{Plan of the paper and summary of results}

In section 2 we review matrix factorisations for B-type boundary conditions in LandauGinzburg models and the variable transformation interface between the Kazama-Suzuki model and products of minimal models. Section 3 gives an introduction to the conformal field theory description of Kazama-Suzuki models. We introduce the rational B-type boundary conditions $|L, \ell\rangle$ (labelled by two integers) in the $\mathrm{SU}(3) / \mathrm{U}(2)$ model and renormalisation group flows between them. Defects $D_{[\Lambda, \Sigma ; \lambda, \mu]}$ (the $\mathrm{SU}(3) / \mathrm{U}(2)$ coset labels are explained in section 3.1) and their fusion to boundary conditions are briefly reviewed.

After these preparations we present in section 4 our central result: a dictionary between all rational boundary conditions $|L, \ell\rangle$ and matrix factorisations $Q_{|L, \ell\rangle}$. We start in section 4.1 from the identification [13] of the boundary conditions $|L, 0\rangle$ with the polynomial matrix factorisations $Q_{|L, 0\rangle}$ (cf. (4.3)). We show that they can also be obtained from permutation factorisations in the product of two minimal models with the help of the variable transformation interface. As presented in section 4.2, the interface allows us to relate the computation of RR-charges in Kazama-Suzuki models to computations in minimal models. One may obtain another class of identifications via taking cones of the factorisations $Q_{|L, 0\rangle}$. The construction principle [13] is prescribed by the Fredenhagen-Schomerus flow rules (4.17), resulting in the explicit formula (4.19) for the factorisations $Q_{|L, 1\rangle}$. As detailed in the second half of section 4.3 , it is not feasible to search for further classes of identifications via the route of taking more complex cones.

In section 4.4 we use the interface to identify the topological defect $D_{[0,0 ; 1,3]}$ in the Landau-Ginzburg description. We identify it in terms of a "fusion functor" $D_{(1)}$ as presented in (4.27), which acts on the polynomial entries of a matrix factorisation and implements the fusion of this defect onto a boundary. From the conformal field theory description we know how this defect acts on rational boundary conditions (see (4.31)), and in this way - starting from the factorisations $Q_{|L, 0\rangle}$ — we have obtained a recursive Ansatz (4.32) to construct all factorisations $Q_{|L, \ell\rangle}$.

The explicit realisation of this Ansatz proves to be technically challenging, yet we succeed in constructing the full set of identifications in closed form. Firstly, we focus in section 4.5.1 on the simpler case of the rational matrix factorisations of type $Q_{|0, \ell\rangle}$, obtaining the closed formula (4.56). With a considerable amount of effort, we then proceed in section 4.5.2 to complete the dictionary. The key to this derivation is the technical observation that the rational factorisations $Q_{|L, 0\rangle}$ may be written as a cone of very simple polynomial factorisations as presented in (4.62). It is then possible to devise an inductive 
proof that ultimately leads to the closed formula (4.76) for the rational factorisations $Q_{|L, \ell\rangle}$. While some elements of the derivation await a rigorous mathematical proof, we provide strong evidence that this is indeed the correct identification, including the highly non-trivial consistency check of the effects of truncation for finite levels $k$ of the Kazama-Suzuki models as presented in section 4.6.

We have collected some of the more technical steps in the appendix.

\section{Matrix factorisations and variable transformation interfaces}

In this section we introduce the description of B-type boundary conditions in LandauGinzburg models as matrix factorisations. We then review the construction of variable transformation interfaces, and discuss in detail the interface between the $\mathrm{SU}(3) / \mathrm{U}(2)$ Kazama-Suzuki model and the product of two minimal models.

\subsection{Matrix factorisations in Landau-Ginzburg models}

B-type boundary conditions in $\mathcal{N}=(2,2)$ supersymmetric Landau Ginzburg models can be described by matrix factorisations $Q$ of the superpotential $W$ (see [7, 15-18]). We want to consider a polynomial superpotential $W\left(x_{1}, \ldots, x_{n}\right)$, and the factorisation $Q$ is then a polynomial square matrix of the form

$$
Q=\left(\begin{array}{cc}
0 & Q^{(1)} \\
Q^{(0)} & 0
\end{array}\right)
$$

such that

$$
Q^{2}=W \cdot \mathbf{1} .
$$

The spectrum of chiral primary boundary fields is encoded in terms of morphisms between matrix factorisations. Let $Q_{1}$ and $Q_{2}$ be two matrix factorisations of size $2 q_{1}$ and $2 q_{2}$, respectively. $Q_{i}$ implements an endomorphism on $R^{2 q_{i}}$, where $R=\mathbb{C}\left[x_{1}, \ldots, x_{n}\right]$ is the polynomial ring in the variables $x_{1}, \ldots, x_{n}$. There is a natural $\mathbb{Z}_{2}$ grading on these free modules, $R^{2 q_{i}}=R^{q_{i}} \oplus R^{q_{i}}$, such that $Q_{i}$ defines an odd map. Also morphisms $\phi_{n}$ between $Q_{1}$ and $Q_{2}$ come with a $\mathbb{Z}_{2}$ degree $n$. They are given by even $(n=0)$ or odd $(n=1)$ homomorphisms from $R^{2 q_{1}}$ to $R^{2 q_{2}}$ that satisfy the closure condition

$$
Q_{2} \phi_{n}-(-1)^{n} \phi_{n} Q_{1}=0 .
$$

In addition, two morphisms that differ by an exact morphism of the form

$$
\tilde{\phi}_{n}=Q_{2} \psi+(-1)^{n} \psi Q_{1}
$$

are identified.

If for two matrix factorisations $Q_{1}, Q_{2}$ there is a homomorphism $\phi_{0}$ between $Q_{1}$ and $Q_{2}$, and a homomorphism $\psi_{0}$ between $Q_{2}$ and $Q_{1}$, such that $\phi_{0} \circ \psi_{0}$ and $\psi_{0} \circ \phi_{0}$ coincide with the identity up to exact terms (2.4), then we say that these two matrix factorisations are equivalent. 
In particular if the factorisations $Q_{1}$ and $Q_{2}$ are of the same size and are related by a similarity transformation $\mathcal{U}$,

$$
Q_{2}=\mathcal{U} \cdot Q_{1} \cdot \mathcal{U}^{-1}
$$

then $Q_{1}$ and $Q_{2}$ are equivalent with $\phi_{0}=\mathcal{U}$ and $\psi_{0}=\mathcal{U}^{-1}$.

Given two factorisations $Q_{1}$ and $Q_{2}$ and an odd morphism $\phi_{1}$ from $Q_{1}$ to $Q_{2}$, one can build a new factorisation $C\left(Q_{1}, Q_{2} ; \phi_{1}\right)$ by the so-called cone construction that is related to the process of tachyon condensation (see e.g. [19, 20]),

$$
C\left(Q_{1}, Q_{2} ; \phi_{1}\right)=\left(\begin{array}{cc}
Q_{1} & 0 \\
\phi_{1} & Q_{2}
\end{array}\right) .
$$

\subsection{Variable transformation interfaces}

We can describe B-type interfaces between Landau-Ginzburg models with superpotentials $W^{y}\left(y_{1}, \ldots, y_{n}\right)$ and $W^{x}\left(x_{1}, \ldots, x_{m}\right)$ by matrix factorisations of the difference $W^{y}-W^{x}$ of the superpotentials [21] (see also [22, 23]). They can be fused to other matrix factorisations by means of the tensor product of matrix factorisations [23, 24].

If the two superpotentials are related to each other by a variable transformation,

$$
y_{j} \mapsto Y_{j}\left(x_{1}, \ldots, x_{m}\right),
$$

that expresses the $y_{j}$ as polynomials in the variables $x_{i}$, such that

$$
W^{x}\left(x_{1}, \ldots, x_{m}\right)=W^{y}\left(Y_{1}\left(x_{1}, \ldots, x_{m}\right), \ldots, Y_{n}\left(x_{1}, \ldots, x_{m}\right)\right),
$$

there is a particular variable transformation interface ${ }_{y} I_{x}$ that we introduced in [14]. It is closely related to the identity defect ${ }_{y} I_{y}$ in the Landau-Ginzburg model with superpotential $W^{y}$, which is given by a particular factorisation

$$
\left({ }_{y} I_{y}\left(y_{1}, \ldots, y_{n} ; y_{1}^{\prime}, \ldots, y_{n}^{\prime}\right)\right)^{2}=W^{y}\left(y_{1}, \ldots, y_{n}\right)-W^{y}\left(y_{1}^{\prime}, \ldots, y_{n}^{\prime}\right)
$$

whose effect on other factorisations by fusion is trivial. Concrete formulas can be found e.g. in [25]. In the case of a two-variable potential the identity defect can be described by the factorisation

$$
{ }_{y} I_{y}=\left(\begin{array}{cccc}
0 & 0 & y_{1}-y_{1}^{\prime} & y_{2}-y_{2}^{\prime} \\
0 & 0 & -\frac{W^{y}\left(y_{1}^{\prime}, y_{2}\right)-W^{y}\left(y_{1}^{\prime}, y_{2}^{\prime}\right)}{y_{2}-y_{2}^{\prime}} & \frac{W^{y}\left(y_{1}, y_{2}\right)-W^{y}\left(y_{1}^{\prime}, y_{2}\right)}{y_{1}-y_{1}^{\prime}} \\
\frac{W^{y}\left(y_{1}, y_{2}\right)-W^{y}\left(y_{1}^{\prime}, y_{2}\right)}{y_{1}-y_{1}^{\prime}} & -\left(y_{2}-y_{2}^{\prime}\right) & 0 & 0 \\
\frac{W^{y}\left(y_{1}^{\prime}, y_{2}\right)-W^{y}\left(y_{1}^{\prime}, y_{2}^{\prime}\right)}{y_{2}-y_{2}^{\prime}} & y_{1}-y_{1}^{\prime} & 0 & 0
\end{array}\right) .
$$

The variable transformation interface ${ }_{y} I_{x}$ is obtained from the identity defect by replacing the variables $y_{j}^{\prime}$ by $Y_{j}(x)$ : this results in a factorisation of $W^{y}\left(y_{1}, \ldots, y_{n}\right)-W^{x}\left(x_{1}, \ldots, x_{m}\right)$. The fusion of this interface to other matrix factorisations can be described in a simple way as we will review in the following. 
If we denote the polynomial rings in $x_{i}$ and $y_{i}$ variables by $S$ and $R$, respectively, the variable transformation (2.7) defines a ring homomorphism $Y$,

$$
Y: R \rightarrow S, \quad Y: p\left(y_{1}, \ldots, y_{n}\right) \mapsto p\left(Y_{1}\left(x_{1}, \ldots, x_{m}\right), \ldots, Y_{n}\left(x_{1}, \ldots, x_{m}\right)\right) .
$$

Using this homomorphism we can view $S$ as an $(S, R)$-bimodule ${ }_{S} S_{R}$ or as an $(R, S)$ bimodule ${ }_{R} S_{S}$. This defines two functors, the extension of scalars $Y^{*}$ maps $R$-modules to $S$-modules by tensoring with ${ }_{S} S_{R}$, and the restriction of scalars $Y_{*}$ maps $S$ - to $R$-modules by tensoring with ${ }_{R} S_{S}$.

Let us discuss the first one, $Y^{*}$, more explicitly. First we observe that this functor maps finite rank free $R$-modules to finite rank free $S$-modules of the same rank,

$$
{ }_{S} S_{R} \otimes_{R}\left({ }_{R} R \oplus \cdots \oplus{ }_{R} R\right) \cong{ }_{S} S \oplus \cdots \oplus{ }_{S} S
$$

A homomorphism between finite rank free $R$-modules, which can be viewed as a matrix with polynomial entries in the variables $y_{i}$, is mapped to the homomorphism between $S$ modules that is obtained by replacing all variables $y_{i}$ by the polynomials $Y_{i}\left(x_{1}, \ldots, x_{m}\right)$. So it acts by replacement of variables: it takes polynomial matrices in variables $y_{j}$ and maps them to polynomial matrices in variables $x_{i}$.

The second one, $Y_{*}$, maps an $S$-module to an $R$-module by tensoring it with ${ }_{R} S_{S}$,

$$
{ }_{S} M \mapsto{ }_{R} S_{S} \otimes_{S S} M
$$

This is in general not a finite rank free $R$-module, even if ${ }_{S} M$ was a finite rank free $S$ module. If on the other hand ${ }_{R} S$ as an $R$-module is free and of finite rank,

$$
\rho:{ }_{R} R^{\oplus r} \stackrel{\sim}{\rightarrow} R S
$$

with $\rho$ an $R$-module isomorphism, then a free $S$-module ${ }_{S} M$ of rank $d$ is mapped to a free $R$-module of rank $r \cdot d$. In this case, its action on homomorphisms can also be described very concretely: given any homomorphism $\phi$ of free $S$-modules of finite rank, we can represent it by a matrix whose entries $\phi_{i j}$ are polynomials in $S$. The homomorphism between the images of the modules under $Y_{*}$ is then described by the matrix that is obtained by replacing each entry $\phi_{i j}$ by a $r \times r$-block that describes the map $\rho^{-1} \circ \phi_{i j} \circ \rho$. Therefore the functor $Y_{*}$ maps matrices in the variables $x_{i}$ to (in general larger) matrices in the variables $y_{j}$.

To summarise, we have introduced two functors that on polynomial entries act as

$$
\begin{aligned}
Y^{*}\left(p\left(y_{1}, \ldots, y_{n}\right)\right) & =p\left(Y_{1}\left(x_{1}, \ldots, x_{m}\right), \ldots, Y_{n}\left(x_{1}, \ldots, x_{m}\right)\right) \\
Y_{*}\left(p\left(x_{1}, \ldots, x_{m}\right)\right) & =\rho^{-1} \circ p \circ \rho .
\end{aligned}
$$

These two functors describe the fusion of the variable transformation interface ${ }_{y} I_{x}$ : fusing it to the left onto a factorisation $Q^{y}$ of $-W^{y}$, it acts by replacement of variables $\left(Q^{y} \mapsto\right.$ $Y^{*}\left(Q^{y}\right)$ ); fusing it to the right onto a factorisation $Q^{x}$ of $W^{x}$, it acts as $Q^{x} \mapsto Y_{*}\left(Q^{x}\right)$. Similarly, we can define an interface ${ }_{x} I_{y}$ whose fusion to the right is described by $Y^{*}$, and whose fusion to the left is described by $Y_{*}$. 
The simplest example of a variable transformation interface is obtained if the rings are the same, $S=R$, and the map $Y=\sigma$ is an automorphism of $R$. In this case, $Y^{*}$ acts by replacing variables according to $Y$, whereas the action of $Y_{*}$ is given by the inverse $Y^{-1}$. In case the two superpotentials are the same, and $\sigma$ is a symmetry of $W$, these interfaces are also known as group-like defects or symmetry defects [21, 25, 26].

\subsection{Kazama-Suzuki models}

We now come to our key example, which will be important for the rest of this paper. These are the Grassmannian Kazama-Suzuki models $\mathrm{SU}(n+1) / \mathrm{U}(n)$, where we will be interested in particular in the case $n=2$.

For general $n \geq 1$, we consider the superpotential

$$
W_{n ; k}^{x}\left(x_{1}, \ldots, x_{n}\right)=x_{1}^{k+n+1}+\cdots+x_{n}^{k+n+1},
$$

where $n, k \geq 1$ are integers. As $W^{x}$ is completely symmetric in $x_{1}, \ldots, x_{n}$, we can express it in terms of the elementary symmetric polynomials

$$
Y_{j}\left(x_{1}, \ldots, x_{n}\right)=\sum_{1 \leq i_{1}<\cdots<i_{j} \leq n} x_{i_{1}} \cdots x_{i_{j}}, \quad j=1, \ldots, n,
$$

to obtain a superpotential $W^{y}$ in variables $y_{1}, \ldots, y_{n}$ such that

$$
W_{n ; k}^{y}\left(Y_{1}\left(x_{1}, \ldots, x_{n}\right), \ldots, Y_{n}\left(x_{1}, \ldots, x_{n}\right)\right)=W_{n ; k}^{x}\left(x_{1}, \ldots, x_{n}\right) .
$$

The superpotential $W^{x}$ describes the tensor product of $n$ minimal models, whereas $W^{y}$ describes the $\mathrm{SU}(n+1) / \mathrm{U}(n)$ Kazama-Suzuki model (see $[5,27])$. We are now precisely in the setup of the previous subsection, and we can define a variable transformation interface ${ }_{y} I_{x}$ between these models. For $n=2$, it is given by the factorisation

$$
\begin{aligned}
& { }_{y} I_{x}= \\
& \left(\begin{array}{cccc}
0 & 0 & y_{1}-x_{1}-x_{2} & y_{2}-x_{1} x_{2} \\
0 & 0 & -\frac{W^{y}\left(x_{1}+x_{2}, y_{2}\right)-W^{x}\left(x_{1}, x_{2}\right)}{y_{2}-x_{1} x_{2}} & \frac{W^{y}\left(y_{1}, y_{2}\right)-W^{y}\left(x_{1}+x_{2}, y_{2}\right)}{y_{1}-x_{1}-x_{2}} \\
\frac{W^{y}\left(y_{1}, y_{2}\right)-W^{y}\left(x_{1}+x_{2}, y_{2}\right)}{y_{1}-x_{1}-x_{2}} & -\left(y_{2}-x_{1} x_{2}\right) & 0 & 0 \\
\frac{W^{y}\left(x_{1}+x_{2}, y_{2}\right)-W^{x}\left(x_{1}, x_{2}\right)}{y_{2}-x_{1} x_{2}} & y_{1}-x_{1}-x_{2} & 0 & 0
\end{array}\right) .
\end{aligned}
$$

It acts on the left just by replacing the variables $y_{j}$ by $Y_{j}\left(x_{1}, \ldots, x_{n}\right)$. To understand its behaviour on the right, i.e. its action on the $x$-variables, we have to understand the structure of $S=\mathbb{C}\left[x_{1}, \ldots, x_{n}\right]$ as a module over $R=\mathbb{C}\left[y_{1}, \ldots, y_{n}\right]$. In the following we want to restrict to the case $n=2$. We choose the explicit $R$-module isomorphism $\rho$ between $R \oplus R$ and $R S$ as

$$
\rho:\left(p_{1}\left(y_{1}, y_{2}\right), p_{2}\left(y_{1}, y_{2}\right)\right) \mapsto p_{1}\left(x_{1}+x_{2}, x_{1} x_{2}\right)+\left(x_{1}-x_{2}\right) p_{2}\left(x_{1}+x_{2}, x_{1} x_{2}\right) .
$$

The inverse is then given by

$$
\rho^{-1}: p\left(x_{1}, x_{2}\right) \mapsto\left(\left.p_{S}\left(x_{1}, x_{2}\right)\right|_{y},\left.\frac{1}{x_{1}-x_{2}} p_{A}\left(x_{1}, x_{2}\right)\right|_{y}\right)
$$


where

$$
p_{S / A}\left(x_{1}, x_{2}\right)=\frac{1}{2}\left(p\left(x_{1}, x_{2}\right) \pm p\left(x_{2}, x_{1}\right)\right),
$$

and for a symmetric polynomial $q\left(x_{1}, x_{2}\right)$ we denote by $\left.q\left(x_{1}, x_{2}\right)\right|_{y}$ the polynomial in $y$ variables from which one obtains $q\left(x_{1}, x_{2}\right)$ when one replaces $y_{i}$ by $Y_{i}\left(x_{1}, x_{2}\right)$.

The functor $Y_{*}$ sends an $S$-module of rank $r$ to an $R$-module of rank $2 r$. On homomorphisms it acts by replacing each polynomial entry by a $2 \times 2$ matrix. With the explicit isomorphism $\rho$ given above, the action of $Y_{*}$ on a polynomial $p\left(x_{1}, x_{2}\right)$ can be determined from (2.16), and it is given by

$$
Y_{*}: p \mapsto\left(\begin{array}{cc}
\left.p_{S}\right|_{y} & \left.\left(x_{1}-x_{2}\right) p_{A}\right|_{y} \\
\left.\frac{p_{A}}{x_{1}-x_{2}}\right|_{y} & \left.p_{S}\right|_{y}
\end{array}\right) .
$$

This variable transformation interface can then be used to relate defects and boundary conditions in Kazama-Suzuki models to those in minimal models. It lies at the heart of the constructions in this paper.

\section{Boundaries and defects in Kazama-Suzuki models}

In this section we review the construction of rational boundary conditions in Grassmannian Kazama-Suzuki model with emphasis on the model based on the coset $\mathrm{SU}(3) / \mathrm{U}(2)$. We also discuss renormalisation group flows of boundary conditions, and topological defects and their fusion to boundaries.

\subsection{Bulk theory}

Kazama-Suzuki models $[3,4]$ are rational $\mathcal{N}=(2,2)$ superconformal field theories that are constructed as cosets

$$
\frac{G_{k} \times S O(d)_{1}}{H},
$$

where $d$ is the difference between the dimension of the simple Lie group $G$ and the dimension of its regularly embedded subgroup $H$. The integer $k$ is the level, and for $\mathcal{N}=(2,2)$ supersymmetry, the geometric space $G / H$ has to be Kähler. A particularly interesting class of such models are the Grassmannian models based on $G=\mathrm{SU}(n+1)$ and $H=\mathrm{U}(n)$, and in this work we specify the model further by considering the case $n=2$.

In the following we briefly review the spectrum of the $\mathrm{SU}(3) / \mathrm{U}(2)$ model. More details can be found e.g. in [13]. The primary fields (w.r.t. the bosonic subalgebra of the chiral symmetry algebra) are labelled by tuples $(\Lambda, \Sigma ; \lambda, \mu)$ where

- $\Lambda=\left(\Lambda_{1}, \Lambda_{2}\right)$ is an $s u(3)$ highest weight $\left(\Lambda_{1}, \Lambda_{2}\right.$ being the non-negative integer Dynkin labels) satisfying $\Lambda_{1}+\Lambda_{2} \leq k$, and it labels a (unitary irreducible) representation of the affine Lie algebra $s u(3)_{k}$,

- $\Sigma \in\{0, v, s, c\}$ labels representations of $s o(4)_{1}$ (with the corresponding representations being the trivial representation, vector, spinor and conjugate spinor), 
- $\lambda$ is a highest weight of $s u(2)$, with $0 \leq \lambda \leq k+1$ labelling a representation of the affine $s u(2)_{k+1}$,

- $\mu$ is an integer modulo $6(k+3)$ labelling representations of $u(1)_{6(k+3)}$.

There is a selection rule on the allowed labels that reads

$$
\frac{\Lambda_{1}+2 \Lambda_{2}}{3}+\frac{|\Sigma|}{2}-\frac{\lambda}{2}+\frac{\mu}{6} \in \mathbb{Z},
$$

where $|\Sigma|=0$ for $\Sigma=0, v$ and $|\Sigma|=1$ for $\Sigma=s, c$. Finally, tuples are identified according to

$$
\left(\left(\Lambda_{1}, \Lambda_{2}\right), \Sigma ; \lambda, \mu\right) \sim\left(\left(k-\Lambda_{1}-\Lambda_{2}, \Lambda_{1}\right), v \times \Sigma ; k+1-\lambda, \mu+(k+3)\right),
$$

where $v \times \cdot$ denotes the fusion with the vector representation, which exchanges on the one hand 0 and $v$, and on the other hand $s$ and $c$.

In the spectrum there are chiral primary fields corresponding to the tuples

$$
\left(\left(\Lambda_{1}, \Lambda_{2}\right), 0 ; \Lambda_{1}, \Lambda_{1}+2 \Lambda_{2}\right)
$$

and they can be labelled by representations $\left(\Lambda_{1}, \Lambda_{2}\right)$ of $s u(3)$.

\subsection{Boundary conditions}

According to how the supercurrents are glued at the boundary of the world-sheet we distinguish between A-type and B-type gluing conditions [28]. Here we are only interested in B-type gluing conditions. Rational boundary conditions can be constructed following the Cardy construction [29]. In the diagonal $\mathrm{SU}(3) / \mathrm{U}(2)$ coset model, maximally symmetric B-type boundary states $|L, S ; \ell\rangle$ are labelled by two integers $L, \ell$ with $0 \leq L \leq\left\lfloor\frac{k}{2}\right\rfloor$, $0 \leq \ell \leq k+1$, and an $s o(4)_{1}$ representation $S$ (see e.g. [13], and also [30] for a general discussion of twisted boundary states in Kazama-Suzuki models). Here, $\lfloor x\rfloor$ denotes the greatest integer smaller or equal $x$. Choosing a particular sign in the gluing condition for the supercurrents, we can restrict to $S=0, v$. We introduce the notation

$$
|L, \ell\rangle:=|L, 0 ; \ell\rangle \quad \text { and } \quad \overline{|L, \ell\rangle}:=|L, v ; \ell\rangle \text {. }
$$

Because of field identifications and selection rules, we have to identify

$$
|L, \ell\rangle \equiv \overline{|L, k+1-\ell\rangle} .
$$

The boundary spectrum is given by $\left(q=e^{2 \pi i \tau}, \tilde{q}=e^{-2 \pi i / \tau}\right)$

$$
\begin{aligned}
& \left\langle L, \ell\left|q^{\frac{1}{2}\left(L_{0}+\bar{L}_{0}\right)-\frac{c}{24}}\right| L^{\prime}, \ell^{\prime}\right\rangle \\
& \quad=\sum_{[\Lambda, \Sigma ; \lambda, \mu]} n_{\Lambda L} L^{L^{\prime}}\left(N_{\lambda \ell}^{(k+1) \ell^{\prime}} \delta_{\Sigma, 0}+N_{\lambda(k+1-\ell)}^{(k+1)}{ }^{\ell^{\prime}} \delta_{\Sigma, v}\right) \chi_{(\Lambda, \Sigma ; \lambda, \mu)}(\tilde{q}) .
\end{aligned}
$$

Here, the sum only goes over equivalence classes of bulk labels, and $N^{(k+1)}$ denotes the fusion rules of $s u(2)_{k+1}, N^{\text {so }}$ the fusion rules of $s o(4)_{1}$, and

$$
n_{\Lambda L} L^{L^{\prime}}=\sum_{\lambda} b_{\lambda}^{\Lambda}\left(N_{\lambda L}^{(k+1) L^{\prime}}-N_{(k+1-\lambda) L}^{(k+1)} L^{\prime}\right)
$$


are twisted fusion rules of $s u(3)_{k}$ (see e.g. [31]). In the last expression the branching rules $b_{\lambda}^{\Lambda}$ of the decomposition of $s u(3)$ representations $\Lambda=\left(\Lambda_{1}, \Lambda_{2}\right)$ into representations $\lambda$ of its regularly embedded subalgebra $s u(2)$ appear. We will later need the branching rules that describe how an $s u(3)$ representation $\left(\Lambda_{1}, \Lambda_{2}\right)$ decomposes into representations $(\lambda ; \mu)$ of $s u(2) \oplus u(1)$,

$$
\left(\Lambda_{1}, \Lambda_{2}\right) \rightarrow \bigoplus_{\lambda, \mu} b_{(\lambda ; \mu)}^{\Lambda}(\lambda ; \mu)=\bigoplus_{\gamma_{1}=0}^{\Lambda_{1}} \bigoplus_{\gamma_{2}=0}^{\Lambda_{2}}\left(\gamma_{1}+\gamma_{2} ; 3\left(\gamma_{1}-\gamma_{2}\right)+2\left(\Lambda_{2}-\Lambda_{1}\right)\right)
$$

From this we directly read off the branching needed in (3.8) by ignoring the $u(1)$ label $\mu$.

\subsection{Boundary renormalisation group flows}

When relevant boundary fields are present, one can study the boundary renormalisation group flows induced by those fields. Such boundary flows have been studied in general cosets in the limit of large levels [32-34]. There is one class of flows that is conjectured to be present at all levels [35-37], which we will briefly describe here.

Applied to the $\mathrm{SU}(3) / \mathrm{U}(2)$ Kazama-Suzuki models, the rule of $[35,36]$ predicts the following renormalisation group flows:

$$
\sum_{\lambda, \ell^{\prime}} b_{\lambda}^{\Lambda^{+}} N_{\lambda \ell}^{(k+1) \ell^{\prime}}\left|L, \ell^{\prime}\right\rangle \longrightarrow \sum_{L^{\prime}} n_{\Lambda L} L^{L^{\prime}}\left|L^{\prime}, \ell\right\rangle
$$

where $\Lambda=\left(\Lambda_{1}, \Lambda_{2}\right)$ is an arbitrary highest weight with $\Lambda_{1}+\Lambda_{2} \leq k$ labelling a representation of $s u(3)_{k}$, and $\Lambda^{+}=\left(\Lambda_{2}, \Lambda_{1}\right)$ is the conjugate representation. $b_{\lambda}^{\Lambda}$ denotes the branching of the $s u(3)$ representation $\Lambda$ into $s u(2)$ representations $\lambda$ (see (3.9)). The field that induces this flow is a linear combination of fields labelled by $((0,0), 0 ; 1, \pm 3)$.

A simple example of such a flow is given by $\Lambda=(1,0)$, and it reads

$$
|L, \ell-1\rangle+|L, \ell\rangle+|L, \ell+1\rangle \longrightarrow \begin{cases}|L-1, \ell\rangle+|L, \ell\rangle+|L+1, \ell\rangle & \text { for } L \neq \frac{k}{2} \\ |L-1, \ell\rangle & \text { for } L=\frac{k}{2}\end{cases}
$$

If a label happens to lie outside the allowed range, the corresponding boundary state has to be omitted (e.g. for $\ell=0$ the first state on the left hand side can be left out).

A nice outcome of this flow rule is that one can obtain all boundary states from a subset of states by perturbing suitable superpositions of boundary states. Successively using the flow (3.11) one can e.g. start from the states $|0, \ell\rangle$ and obtain all others.

\subsection{Defects and fusion}

We can also study topological defects in these models, and here we will focus on defects with B-type gluing conditions for the supercurrents. The rational defects carry the same labels as the bulk fields, $D_{[\Lambda, \Sigma ; \lambda, \mu]}[38]$, and as defect operators on the bulk Hilbert space 
they are given as

$$
\begin{aligned}
D_{[\Lambda, \Sigma ; \lambda, \mu]} & =\sum_{\left[\Lambda^{\prime}, \Sigma^{\prime} ; \lambda^{\prime}, \mu^{\prime}\right]} \frac{S_{[\Lambda, \Sigma ; \lambda, \mu]\left[\Lambda^{\prime}, \Sigma^{\prime} ; \lambda^{\prime}, \mu^{\prime}\right]}^{\mathrm{SU}(3) \mathrm{U}(2)}}{S_{[0,0 ; 0,0]\left[\Lambda^{\prime}, \Sigma^{\prime} ; \lambda^{\prime}, \mu^{\prime}\right]}^{\mathrm{SU}(3) / \mathrm{U}(2)}} P_{\left[\Lambda^{\prime}, \Sigma^{\prime} ; \lambda^{\prime}, \mu^{\prime}\right] \otimes\left[\Lambda^{\prime}, \Sigma^{\prime} ; \lambda^{\prime}, \mu^{\prime}\right]} \\
& =\sum_{\left[\Lambda^{\prime}, \Sigma^{\prime} ; \lambda^{\prime}, \mu^{\prime}\right]} \frac{S_{\Lambda \Lambda^{\prime}}^{\mathrm{SU}(3)} S_{\Sigma \Sigma^{\prime}}^{\mathrm{SO}(4)} \bar{S}_{\lambda \lambda^{\prime}}^{\mathrm{SU}(2)} \bar{S}_{\mu \mu^{\prime}}^{\mathrm{U}(1)}}{S_{0 \Lambda^{\prime}}^{\mathrm{SU}(3)} S_{0 \Sigma^{\prime}}^{\mathrm{SO}(4)} \bar{S}_{0 \lambda^{\prime}}^{\mathrm{SU}(2)} \bar{S}_{0 \mu^{\prime}}^{\mathrm{U}(1)}} P_{\left[\Lambda^{\prime}, \Sigma^{\prime} ; \lambda^{\prime}, \mu^{\prime}\right] \otimes\left[\Lambda^{\prime}, \Sigma^{\prime} ; \lambda^{\prime}, \mu^{\prime}\right]},
\end{aligned}
$$

where $S^{\mathrm{SU}(3) / \mathrm{U}(2)}$ is the modular S-matrix of the model, which can be expressed in terms of the modular S-matrices $S^{\mathrm{SU}(3)}, S^{\mathrm{SO}(4)}, S^{\mathrm{SU}(2)}$ and $S^{\mathrm{U}(1)}$ of the constituents. Concrete formulae can be found e.g. in the appendix of [39]. $P_{\left[\Lambda^{\prime}, \Sigma^{\prime} ; \lambda^{\prime}, \mu^{\prime}\right] \otimes\left[\Lambda^{\prime}, \Sigma^{\prime} ; \lambda^{\prime}, \mu^{\prime}\right]}$ is the projection operator onto the corresponding bulk sector. By fixing the sign in the gluing condition for the supercurrents we can restrict the set of defects to those with $\Sigma=0, v$.

Topological defects can be fused to boundaries [38, 40]. Using a B-type defect, a B-type boundary condition is transformed into a superposition of B-type boundary conditions,

$$
D_{[\Lambda, 0 ; \lambda, \mu]}|L, \ell\rangle=\sum n_{\Lambda L} L^{\prime} N_{\lambda \ell}^{(k+1) \ell^{\prime}}\left|L^{\prime}, \ell^{\prime}\right\rangle
$$

Defects that only differ in the label $\mu$ have an identical effect on B-type boundary conditions.

As an example consider the defect $D_{[(0,0), 0 ; 1,3]}$, which is given explicitly by

$$
D_{[(0,0), 0 ; 1,3]}=\sum_{\left[\Lambda^{\prime}, \Sigma^{\prime} ; \lambda^{\prime}, \mu^{\prime}\right]} \frac{\sin \frac{2 \pi\left(\lambda^{\prime}+1\right)}{k+3}}{\sin \frac{\pi\left(\lambda^{\prime}+1\right)}{k+3}} e^{\frac{i \pi}{k+3} \mu^{\prime}} P_{\left[\Lambda^{\prime}, \Sigma^{\prime} ; \lambda^{\prime}, \mu^{\prime}\right] \otimes\left[\Lambda^{\prime}, \Sigma^{\prime} ; \lambda^{\prime}, \mu^{\prime}\right]} .
$$

Fusing this defect to boundary conditions is described by

$$
D_{[(0,0), 0 ; 1,3]}|L, \ell\rangle=|L, \ell+1\rangle+|L, \ell-1\rangle,
$$

where the last boundary condition is omitted if $\ell=0$. Therefore, starting from $|L, 0\rangle$ one can generate all other boundary conditions by fusing $D_{[(0,0), 0 ; 1,3]}$.

\section{Matrix factorisations for rational boundary conditions}

In this section we want to discuss matrix factorisations of the Landau-Ginzburg superpotential $W_{2 ; k}^{y}$ that leads to the $\mathrm{SU}(3) / \mathrm{U}(2)$ Kazama-Suzuki model. In particular we want to identify those factorisations that correspond to rational boundary conditions in the conformal field theory.

We first review the identification of some of the rational boundary conditions as polynomial factorisations (i.e. where the matrix factorisations $Q$ are $2 \times 2$-matrices) [13], and how one can obtain some higher factorisations via the cone construction. Then we will discuss how one can employ defects for a systematic construction of all matrix factorisations corresponding to rational boundary conditions. 


\subsection{Polynomial factorisations}

The superpotential of the $\mathrm{SU}(3) / \mathrm{U}(2)$ Kazama-Suzuki model is given by

$$
\begin{aligned}
W_{2 ; k}^{y}\left(y_{1}, y_{2}\right)= & \left.\left(x_{1}^{k+3}+x_{2}^{k+3}\right)\right|_{\begin{array}{l}
x_{1}+x_{2} \mapsto y_{1} \\
x_{1} x_{2} \mapsto y_{2}
\end{array}}=\left.\prod_{j=0}^{k+2}\left(x_{1}-\eta^{2 j+1} x_{2}\right)\right|_{\begin{array}{l}
x_{1}+x_{2} \mapsto y_{1} \\
x_{1} x_{2} \mapsto y_{2}
\end{array}} \\
= & \prod_{j=0}^{\left\lfloor\frac{k+1}{2}\right\rfloor}\left(y_{1}^{2}-\delta_{j} y_{2}\right) \cdot \begin{cases}y_{1} \text { for } k \text { even } \\
1 & \text { for } k \text { odd },\end{cases}
\end{aligned}
$$

where

$$
\eta=e^{i \pi /(k+3)}, \quad \delta_{j}=\frac{\left(1+\eta^{2 j+1}\right)^{2}}{\eta^{2 j+1}}
$$

The product form of the superpotential allows us to easily write down factorisations $Q^{(1)}$. $Q^{(0)}=W_{2 ; k}^{y}$ with polynomials $Q^{(1)}$ and $Q^{(0)}$. Among those polynomial factorisations we could identify in [13] those that correspond to rational boundary conditions. One class that can be identified in this way consists of the boundary conditions $|L, 0\rangle$, and the associated factorisations are

$$
Q_{|L, 0\rangle}=\left(\begin{array}{cc}
0 & \mathcal{J}_{|L, 0\rangle} \\
\mathcal{J}_{\overline{|L, 0\rangle}} & 0
\end{array}\right)
$$

with

$$
\mathcal{J}_{|L, 0\rangle}=\prod_{j=0}^{L} \mathcal{J}_{j}, \quad \mathcal{J}_{j}=y_{1}^{2}-\delta_{j} y_{2}, \quad \mathcal{J}_{\overline{|L, 0\rangle}}=\frac{W_{2 ; k}^{y}}{\mathcal{J}_{|L, 0\rangle}}
$$

The identification in [13] is based on the comparison of the spectra of chiral primary fields, and of the RR-charges.

For even $k$ there is another class of rational boundary conditions that have a description in terms of polynomial factorisations. These are the boundary conditions $\left|\frac{k}{2}, \ell\right\rangle-$ details can be found in [13].

In section 2.3 we introduced the variable transformation interface ${ }_{y} I_{x}$ between the $\mathrm{SU}(3) / \mathrm{U}(2)$ Kazama-Suzuki model and two copies of minimal models at level $k+1$. Let us briefly discuss how one can obtain the factorisations $Q_{|L, 0\rangle}$ in the Kazama-Suzuki model from factorisations in the product of minimal models by interface fusion. The simplest factorisations in the product of two minimal models are the polynomial factorisations, which are called permutation factorisations [10] (see also [41, 42]). A subset of those corresponds to rational boundary states, namely the permutation boundary states $|L, M\rangle_{\text {perm }}$, which are labelled by two numbers, $L=0, \cdots, k+1$ and $M$ being an integer identified modulo 
$2 k+6$, such that $L+M$ is even. In [10] these have been identified with the factorisations

$$
\begin{aligned}
Q_{|L, M\rangle_{\text {perm }}} & =\left(\begin{array}{cc}
0 & Q_{|L, M\rangle_{\text {perm }}}^{(1)} \\
Q_{|L, M\rangle_{\text {perm }}}^{(0)} & 0
\end{array}\right) \\
& =\left(\begin{array}{cc}
0 & \prod_{j=-\frac{M+L}{2}-1}^{-\frac{M-L}{2}-1}\left(x_{1}-\eta^{2 j+1} x_{2}\right) \\
\prod_{-\frac{M-L}{2}}^{k+1-\frac{M+L}{2}}\left(x_{1}-\eta^{2 j+1} x_{2}\right) & 0
\end{array}\right) .
\end{aligned}
$$

Let us now fuse the interface ${ }_{y} I_{x}$ onto the factorisation $Q_{|2 L, 0\rangle_{\mathrm{perm}}}$. We first note that we can rewrite the product that appears in $Q_{|2 L, 0\rangle_{\text {perm }}}^{(1)}$ as

$$
Q_{|2 L, 0\rangle_{\mathrm{perm}}}^{(1)}=\left.\left(x_{1}-\eta^{-2 L-1} x_{2}\right) \prod_{j=0}^{L-1} \mathcal{J}_{j}\left(y_{1}, y_{2}\right)\right|_{\substack{y_{1} \\ y_{2} \mapsto x_{1}+x_{2}}} .
$$

The effect of fusing ${ }_{y} I_{x}$ is given by the functor $Y_{*}$ defined in (2.24). When we apply it to $Q_{|2 L, 0\rangle_{\text {perm }}}^{(1)}$, we obtain

$$
\begin{aligned}
Y_{*}\left(Q_{|2 L, 0\rangle_{\mathrm{perm}}}^{(1)}\right) & =\prod_{j=0}^{L-1} \mathcal{J}_{j}\left(y_{1}, y_{2}\right) \cdot\left(\begin{array}{cc}
\frac{1}{2}\left(1-\eta^{-L-1}\right) y_{1} & \frac{1}{2}\left(1+\eta^{-L-1}\right)\left(y_{1}^{2}-4 y_{2}\right) \\
\frac{1}{2}\left(1+\eta^{-L-1}\right) & \frac{1}{2}\left(1-\eta^{-L-1}\right) y_{1}
\end{array}\right) \\
& \rightarrow\left(\begin{array}{cc}
\prod_{j=0}^{L} \mathcal{J}_{j}\left(y_{1}, y_{2}\right) & 0 \\
0 & \prod_{j=0}^{L-1} \mathcal{J}_{j}\left(y_{1}, y_{2}\right)
\end{array}\right)
\end{aligned}
$$

where we performed a similarity transformation in the second step. We thus see that

$$
{ }_{y} I_{x} \otimes Q_{|2 L, 0\rangle_{\text {perm }}} \cong Y_{*}\left(Q_{|2 L, 0\rangle_{\text {perm }}}\right) \cong Q_{|L, 0\rangle} \oplus Q_{|L-1,0\rangle},
$$

where it is understood that $Q_{|L-1,0\rangle}$ is absent when $L=0$.

\section{$4.2 \quad$ RR-charges}

The interface ${ }_{y} I_{x}$ between the $\mathrm{SU}(3) / \mathrm{U}(2)$ Kazama-Suzuki model and the two minimal models can also be used to relate correlators in these theories. As a simple example we study the RR-charge, which can be considered as a disc one-point function of the corresponding RR-field.

The chiral primaries in the $\mathrm{SU}(3) / \mathrm{U}(2)$ Kazama-Suzuki model are labelled by $\mathrm{SU}(3)$ representations with Dynkin labels $\left(\Lambda_{1}, \Lambda_{2}\right)$ (see (3.4)) and can be expressed as polynomials in the variables $y_{1}, y_{2}$ (see e.g. [13]),

$$
\Phi_{\left(\Lambda_{1}, \Lambda_{2}\right)}\left(y_{1}, y_{2}\right)=\sum_{r=0}^{\left\lfloor\Lambda_{1} / 2\right\rfloor}(-1)^{r}\left(\begin{array}{c}
\Lambda_{1}-r \\
r
\end{array}\right) y_{1}^{\Lambda_{1}-2 r} y_{2}^{\Lambda_{2}+r} .
$$


The chiral primary fields are related to the Ramond ground states by spectral flow. Only the Ramond ground states with zero $\mathrm{U}(1)_{R}$-charge have non-trivial one-point functions in the presence of a B-type boundary condition. The corresponding chiral primary fields are given by $\Phi_{(k-2 j, j)}$ with $j=0, \ldots,\left\lfloor\frac{k}{2}\right\rfloor$. The one-point function in the presence of the factorisation $Q_{|L, 0\rangle}$ can be derived using the Kapustin-Li formula [18, 43], and it is given by $(\text { see }[13])^{1}$

$$
\left\langle\Phi_{(k-2 j, j)}\right\rangle_{|L, 0\rangle}=-\sum_{i=0}^{L}\left(\eta^{(2 i+1)(j+1)}+\eta^{-(2 i+1)(j+1)}\right) .
$$

On the other hand in the minimal models, the chiral primary fields corresponding to chargeless Ramond ground states are labelled by

$$
\Psi_{j}\left(x_{1}, x_{2}\right)=x_{1}^{j} x_{2}^{k+1-j}
$$

In the presence of a boundary given by the factorisation $Q_{|L, M\rangle_{\text {perm }}}$, one can straightforwardly compute the RR one-point function using again the Kapustin-Li formula [18, 43], and one finds

$$
\left\langle\Psi_{j}\right\rangle_{|L, M\rangle_{\text {perm }}}=\sum_{i=-\frac{M+L}{2}-1}^{-\frac{M-L}{2}-1} \eta^{(2 i+1)(j+1)} .
$$

What is the relation between the RR-charges in the two theories? We observed before (see (4.8)) that the interface ${ }_{y} I_{x}$ maps $|2 L, 0\rangle_{\text {perm }}$ to $|L, 0\rangle \oplus|L-1,0\rangle$. We can therefore interpret the disc one-point function of $\Phi_{(k-2 j, j)}$ in the presence of the boundary condition $|L, 0\rangle \oplus|L-1,0\rangle$ as a limit of the disc correlator with one insertion of $\Phi_{(k-2 j, j)}$ surrounded by the interface ${ }_{y} I_{x}$ and with the minimal model boundary condition $|2 L, 0\rangle_{\text {perm }}$ at the boundary of the disc. We can take the opposite limit of shrinking the interface around the bulk insertion, this will produce some minimal model field $\tilde{\Phi}_{(k-2 j, j)}$. We conclude that ${ }^{2}$

$$
\left\langle\Phi_{(k-2 j, j)}\right\rangle_{|L, 0\rangle \oplus|L-1,0\rangle}=\left\langle\tilde{\Phi}_{(k-2 j, j)}\right\rangle_{|2 L, 0\rangle_{\text {perm }}} .
$$

This is illustrated in figure 1. To check this relation we need to determine the field $\tilde{\Phi}_{(k-2 j, j)}$. In $[46,47]$ it has been worked out how an interface acts on a bulk field. Applying these methods one can see that the action of a variable transformation interface ${ }_{x} I_{y}$ on a field $\Phi\left(y_{j}\right)$ is in general given by ${ }^{3}$

$$
\tilde{\Phi}\left(x_{i}\right)=\operatorname{det}\left(\frac{\partial Y_{r}}{\partial x_{s}}\right) \Phi\left(Y_{j}\left(x_{i}\right)\right)
$$

\footnotetext{
${ }^{1}$ Notice that the expression here differs from the one in [13] by a sign, which is only a matter of convention regarding the definition of the one-point function.

${ }^{2}$ Similar computations have appeared for (generalised) orbifolds of Landau-Ginzburg models in [44, 45].

${ }^{3}$ This result is obtained by applying formula (1.4) of [46] and making use of the fact that the variable transformation interface is obtained from the identity defect ${ }_{y} I_{y}$ by replacing one set of $y$-variables by the corresponding expression in $x$-variables.
} 

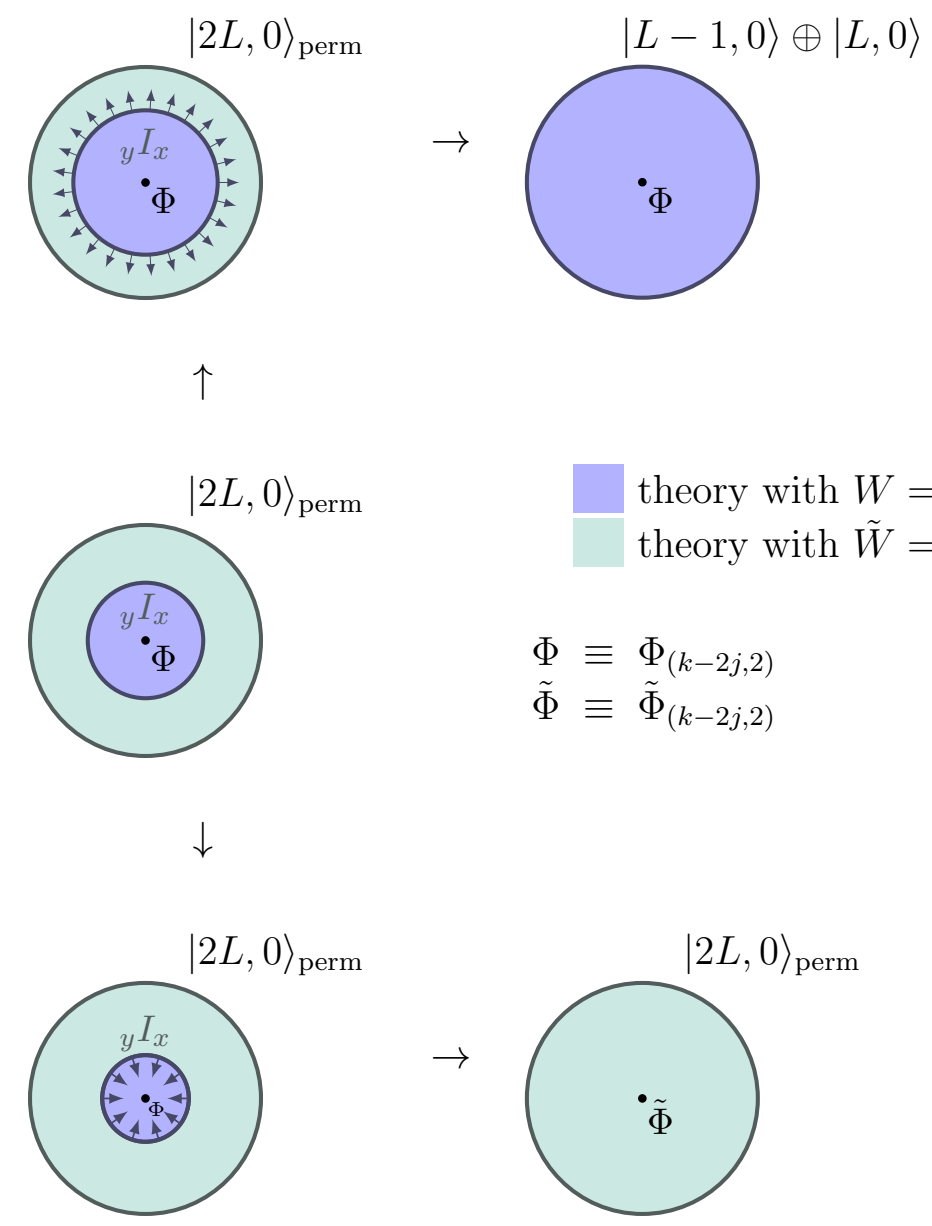

Figure 1. Consider a disc correlator with a bulk field $\Phi$ inserted at the centre, and the interface ${ }_{y} I_{x}$ inserted around it (see the central illustration to the left). Then we can either shrink the interface around the insertion to produce a field insertion by a field $\tilde{\Phi}$, or we let the interface cycle grow until it hits the boundary to produce a new boundary condition. In this way we can relate two bulk one-point functions on the disc.

In our case we obtain

$$
\begin{aligned}
\tilde{\Phi}_{(k-2 j, j)}\left(x_{1}, x_{2}\right) & =\left(x_{1}-x_{2}\right) \cdot \Phi_{(k-2 j, j)}\left(x_{1}+x_{2}, x_{1} x_{2}\right) \\
& =\left(x_{1}-x_{2}\right) \sum_{i=0}^{k-2 j} x_{1}^{i+j} x_{2}^{k-i-j} \\
& =x_{1}^{k-j+1} x_{2}^{j}-x_{1}^{j} x_{2}^{k-j+1} \\
& =\Psi_{k-j+1}\left(x_{1}, x_{2}\right)-\Psi_{j}\left(x_{1}, x_{2}\right)
\end{aligned}
$$


Therefore the right hand side of (4.13) evaluates to

$$
\begin{aligned}
\left\langle\tilde{\Phi}_{(k-2 j, j)}\right\rangle_{|2 L, 0\rangle_{\text {perm }}} & =\sum_{i=-L-1}^{L-1}\left(\eta^{(2 i+1)(k+2-j)}-\eta^{(2 i+1)(j+1)}\right) \\
& =-\sum_{i=-L-1}^{L-1}\left(\eta^{-(2 i+1)(j+1)}+\eta^{(2 i+1)(j+1)}\right) \\
& =-\sum_{i=0}^{L-1}\left(\eta^{(2 i+1)(j+1)}+\eta^{-(2 i+1)(j+1)}\right)-\sum_{i=0}^{L}\left(\eta^{(2 i+1)(j+1)}+\eta^{-(2 i+1)(j+1)}\right),
\end{aligned}
$$

which precisely equals the left hand side of (4.13).

\subsection{Higher factorisations from cones}

To construct matrix factorisations for other rational boundary conditions, one can make use of the known flows between different boundary states [13], which we will review in this subsection. It turns out that this method seems not to be suitable to obtain all those factorisations, but it leads to a precise construction for the factorisations corresponding to the boundary states $|L, 1\rangle$. These in turn constitute the starting point for the identification of a defect factorisation in section 4.4 which will be our main tool to generate factorisations for all rational boundary states in this article.

Evaluating the flow (3.11) for $\ell=0$, we obtain

$$
|L, 0\rangle+|L, 1\rangle \longrightarrow|L-1,0\rangle+|L, 0\rangle+|L+1,0\rangle .
$$

An analogous statement in terms of matrix factorisations entails that the factorisation corresponding to the right hand side can be obtained as a cone from the two factorisations that correspond to the left hand side of the flow (4.17). In other words, $Q_{|L, 1\rangle}$ can be obtained as a cone from $Q_{|L, 0\rangle}$ and the superposition $Q_{|L-1,0\rangle} \oplus Q_{|L, 0\rangle} \oplus Q_{|L+1,0\rangle}$. This in turn can be rewritten [13] as a cone of $Q_{|L, 0\rangle}$ and the factorisation

$$
\tilde{Q}_{L}=\left(\begin{array}{cc}
0 & \mathcal{J}_{L+1} \mathcal{J}_{|L-1,0\rangle} \\
\mathcal{J}_{L} \mathcal{J}_{\overline{|L+1,0\rangle}} & 0
\end{array}\right) .
$$

Explicitly we find

$$
Q_{|L, 1\rangle}=C\left(Q_{|L, 0\rangle}, \tilde{Q}_{L}, y_{1}\left(\begin{array}{cc}
0 & \mathcal{J}_{|L-1,0\rangle} \\
-\mathcal{J}_{|L+1,0\rangle} & 0
\end{array}\right)\right)
$$

such that

$$
\begin{aligned}
Q_{|L, 1\rangle}^{(1)} & =\left(\begin{array}{cc}
\mathcal{J}_{L} & 0 \\
y_{1} & \mathcal{J}_{L+1}
\end{array}\right) \mathcal{J}_{|L-1,0\rangle} \\
Q_{|L, 1\rangle}^{(0)} & =\left(\begin{array}{cc}
\mathcal{J}_{L+1} & 0 \\
-y_{1} & \mathcal{J}_{L}
\end{array}\right) \mathcal{J}_{\overline{|L+1,0\rangle}}
\end{aligned}
$$


This factorisation is the basic input that we need to identify the defect $D_{[0,0 ; 1,3]}$ in the subsequent subsection, from which we will generate factorisations for all other rational boundary states. In the remainder of this subsection, we discuss why it seems difficult to continue the strategy of building cones to construct factorisations for boundary states $|L, \ell\rangle$ with $\ell \geq 2$ directly.

There are two obstacles in this approach, one of a technical nature, the other one being a conceptual problem. On the technical side one faces the problem that the factorisations in question become larger and larger, and the computations are feasible only by means of a computer program. In fact, the flow rule (4.17) leads to a realisation of $Q_{|L, 1\rangle}$ as an $8 \times 8$ matrix (that can then be reduced to the $4 \times 4$ matrix that we saw above), and similarly the general flow rule (3.11) leads to an ansatz where the $Q_{|L, 2\rangle}$ factorisations are already $32 \times 32$ matrices, and the $Q_{|L, 3\rangle}$ are of size $128 \times 128$. Even with the help of rather efficient SINGULAR codes and considerable amounts of computer processing power, the authors were not able to push this type of search much beyond the $Q_{|L, 2\rangle}$ type factorisations, with only a few sporadic matches for $Q_{|L, 3\rangle}$, and the codes not being executable due to memory limitations already for the $Q_{|L, 4\rangle}$ type factorisations.

There is also a conceptual problem in this approach. For the $|L, 1\rangle$ boundary states, one can uniquely identify the field that is responsible for the flow by its $\mathrm{U}(1)_{R}$-charge, and therefore one is led to a unique ansatz for the cone. This is in general not true for $|L, 2\rangle$ and beyond. This problem is also reflected by the presence of marginal boundary fields for the $|L, 2\rangle$ boundary condition (if $L \neq k / 2$ ): it can be smoothly deformed to other boundary states. Correspondingly, the associated matrix factorisations can be deformed, and within this continuous family of $|L, 2\rangle$-like factorisations it is hard to identify the one that corresponds precisely to $|L, 2\rangle$.

This is why we look for a different approach to obtain the higher factorisations, which will be based on special operator-like defects in the theory as we will discuss in the following.

\subsection{Higher factorisations from defect fusion}

Besides the cone construction, which we employed in the last subsection, we can also use fusion of defects or interfaces to generate new factorisations. We have seen in section 4.1 that we can generate the $|L, 0\rangle$ factorisations from permutation factorisations in minimal models by fusing the variable transformation interface ${ }_{y} I_{x}$, namely

$$
{ }_{y} I_{x} \otimes Q_{|2 L, 0\rangle_{\mathrm{perm}}} \cong Y_{*}\left(Q_{|2 L, 0\rangle_{\mathrm{perm}}}\right) \cong Q_{|L-1,0\rangle} \oplus Q_{|L, 0\rangle}
$$

What happens if we tensor ${ }_{y} I_{x}$ to other permutation factorisations? Let us look at the factorisations corresponding to the permutation boundary states $|2 L+1,1\rangle_{\text {perm }}$. From (4.5) we see that the upper right entry is

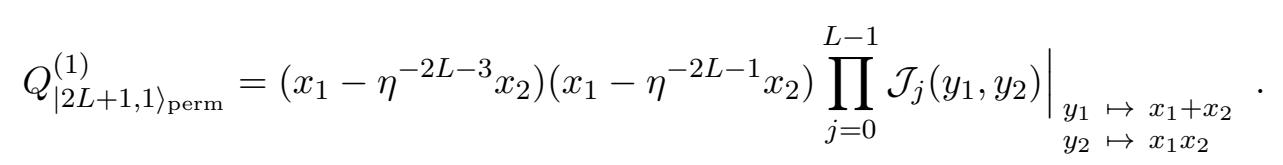


Fusing the variable transformation interface to this factorisation, i.e. applying the functor $Y_{*}$, we obtain

$$
\begin{aligned}
& Y_{*}\left(Q_{|2 L+1,1\rangle_{\mathrm{perm}}}^{(1)}\right)=\prod_{j=0}^{L-1} \mathcal{J}_{j}\left(y_{1}, y_{2}\right) \times \\
& \times\left(\begin{array}{cc}
\frac{1+\eta^{-4 L-4}}{2} y_{1}^{2}-\left(1-\eta^{-2 L-1}\right)\left(1-\eta^{-2 L-3}\right) & \frac{1-\eta^{-2 L-4}}{2} y_{1}\left(y_{1}^{2}-4 y_{2}\right) \\
\frac{1-\eta^{-2 L-4}}{2} y_{1} & \frac{1+\eta^{-4 L-4}}{2} y_{1}^{2}-\left(1-\eta^{-2 L-1}\right)\left(1-\eta^{-2 L-3}\right)
\end{array}\right) \\
& \rightarrow\left(\begin{array}{cc}
\mathcal{J}_{L} & 0 \\
y_{1} & \mathcal{J}_{L+1}
\end{array}\right) \mathcal{J}_{|L-1,0\rangle},
\end{aligned}
$$

where we performed a similarity transformation in the last step. This is precisely $Q_{|L, 1\rangle}^{(1)}$ (see (4.20a)), so that we find

$$
Y_{*}\left(Q_{|2 L+1,1\rangle_{\text {perm }}}\right) \cong Q_{|L, 1\rangle}
$$

We found again that a rational boundary condition is mapped to a rational one by the variable transformation interface.

There is, however, much more that we can conclude from this finding. In fact we expect from the rational conformal field theory description that there is a defect $D_{[(0,0), 0 ; 1,3]}$ that maps $|L, 0\rangle$ to $|L, 1\rangle$. A natural ansatz would be to look for a rational defect $\tilde{D}$ in the minimal model theory, and then fusing it from the left with ${ }_{y} I_{x}$ and from the right with ${ }_{x} I_{y}$ to obtain a defect in the Kazama-Suzuki model,

$$
D_{[(0,0), 0 ; 1,3]}={ }_{y} I_{x} \otimes \tilde{D} \otimes{ }_{x} I_{y} .
$$

We know that under fusion with $D_{[(0,0), 0 ; 1,3]}$ the factorisation $Q_{|L, 0\rangle}$ should be mapped to $Q_{|L, 1\rangle}$. Fusing the variable transformation interface onto $Q_{|L, 0\rangle}$ leads to the factorisation $Q_{|2 L+1,-1\rangle_{\text {perm }}}$. On the other hand we just derived that $Q_{|2 L+1,1\rangle_{\text {perm }}}$ is mapped to $Q_{|L, 1\rangle}$ when we fuse ${ }_{y} I_{x}$. Therefore we demand that the defect $\tilde{D}$ maps $|2 L+1,-1\rangle_{\text {perm }}$ to $\mid 2 L+$ $1,1\rangle_{\text {perm. }}$. In fact there is a symmetry defect, $Q_{\{1\}} \otimes Q_{\left\{\eta^{2}\right\}}$ that acts as the identity defect in the first minimal model factor, and as the symmetry defect realising the automorphism $\sigma_{\eta^{2}}: x_{2} \rightarrow \eta^{2} x_{2}$ in the second minimal model. We therefore conjecture that

$$
\tilde{D}=Q_{\{1\}} \otimes Q_{\left\{\eta^{2}\right\}}
$$

This is again a simple example of a variable transformation interface, whose fusion is described by the functor $\sigma_{\eta^{2}}^{*}$ that acts trivially on the variable $x_{1}$ and replaces the variable $x_{2}$ by $\eta^{2} x_{2}$. The fusion of the defect $D_{[(0,0), 0 ; 1,3]}$ can then be described by the functor

$$
D_{(1)}=Y_{*} \circ \sigma_{\eta^{2}}^{*} \circ Y^{*} .
$$

We have thus identified a candidate for a defect in the Landau-Ginzburg theory whose action on the boundary conditions $Q_{|L, 0\rangle}$ coincides precisely with what we expect from the fusion of the defect $D_{[(0,0), 0 ; 1,3]}$ on the boundary condition $|L, 0\rangle$. This is of course not a 
proof that we identified the defect correctly in the Landau-Ginzburg model, and we briefly want to discuss two obvious ways how one could try to modify the proposal. Firstly we might modify the proposal by choosing instead of $Q_{\{1\}} \otimes Q_{\eta^{2}}$ the symmetry defect

$$
Q_{\left\{\eta^{2 m}\right\}} \otimes Q_{\left\{\eta^{2(m+1)}\right\}}
$$

which would lead to the same action on boundary conditions $Q_{|L, 0\rangle}$. To decide which choice is the correct one, we have to act with the defect on other defects. From the conformal field theory we expect the fusion

$$
D_{[(0,0), 0 ; 1,3]} * D_{[(0,0), 0 ; 1,3]}=D_{[(0,0), 0 ; 2,6]} \oplus D_{[(0,0), 0 ; 0,6]} .
$$

The second defect is a symmetry defect that corresponds to the phase shifts

$$
y_{1} \mapsto \eta^{2} y_{1}, y_{2} \mapsto \eta^{4} y_{2}
$$

which means that we know its identification on the Landau-Ginzburg side. By looking at the above fusion of the defect $D_{[(0,0), 0 ; 1,3]}$ with itself in the Landau-Ginzburg theory (which we will present in [48]), we can therefore confirm that we made the correct choice.

The second obvious question one should investigate is whether there are any smooth deformations of this defect, so that there would be a whole family of defects with similar properties. As one can show from a computation of the conformal field theory spectrum, we do not expect any fermionic morphisms of the corresponding matrix factorisations, and therefore no deformations. This provides further evidence that we have identified the defect correctly.

Having identified $D_{[(0,0), 0 ; 1,3]}$ in the Landau-Ginzburg model, one can then use it to construct higher factorisations, which we will do in the following subsection.

\subsection{Matrix factorisations for all rational boundary conditions}

With the help of the defect $D_{[(0,0), 0 ; 1,3]}$ we can in principle determine all matrix factorisations corresponding to rational boundary conditions. In fact, we know from the conformal field theory that (see (3.16))

$$
D_{[(0,0), 0 ; 1,3]}|L, \ell\rangle=|L, \ell-1\rangle+|L, \ell+1\rangle,
$$

where it is understood that the first boundary condition on the right is not present for $\ell=0$. For the factorisations this means that

$$
D_{(1)}\left(Q_{|L, \ell\rangle}\right) \cong Q_{|L, \ell-1\rangle} \oplus Q_{|L, \ell+1\rangle}
$$

Starting from $Q_{|L, 0\rangle}$ one can generate all $Q_{|L, \ell\rangle}$ by successively applying $D_{(1)}$. The technical challenge that remains is to decompose the fusion result into the direct sum of two factorisations. 


\subsubsection{A closed formula for rational matrix factorisations $Q_{|0, \ell\rangle}$}

We now want to investigate this problem for the factorisations of type $Q_{|0, \ell\rangle}$. They are generated from the factorisation $Q_{|0,0\rangle}$ which is a $2 \times 2$ matrix whose upper right block $Q_{|0,0\rangle}^{(1)}$ is the polynomial $\mathcal{J}_{0}$ (see (4.3)). Applying $D_{(1)}$ once we obtain a matrix factorisation for $|0,1\rangle$ whose upper right block (after a similarity transformation) is given by (see (4.20a))

$$
D_{(1)}\left(\mathcal{J}_{0}\right) \cong Q_{|0,1\rangle}^{(1)}=\left(\begin{array}{cc}
\mathcal{J}_{0} & 0 \\
y_{1} & \mathcal{J}_{1}
\end{array}\right)
$$

We see the polynomial factors $\mathcal{J}_{n}$ appearing on the diagonal. In the full matrix factorisation $Q_{|0,1\rangle}$ they appear as part of the matrix factorisation blocks

$$
Q_{n}=\left(\begin{array}{cc}
0 & \mathcal{J}_{n} \\
\overline{\mathcal{J}}_{n} & 0
\end{array}\right)
$$

with $\overline{\mathcal{J}}_{n}=W_{2 ; k}^{y} / \mathcal{J}_{n}$.

When we want to apply $D_{(1)}$ once more, we first have to understand its action on these blocks $Q_{n}$. We will need later a result not only for $Q_{0}$ and $Q_{1}$, but for a general factorisation $Q_{n}$. Introducing the notation

$$
\pi_{p}=\frac{1}{2}\left(1+\eta^{p}\right) \quad \mu_{p}=\frac{1}{2}\left(1-\eta^{p}\right),
$$

the factor $\mathcal{J}_{n}$ (see (4.4)) can be expressed as

$$
\mathcal{J}_{n}=y_{1}^{2} \mu_{2 n+1} \mu_{-2 n-1}+\lambda_{1}^{2} \pi_{2 n+1} \pi_{-2 n-1},
$$

where

$$
\lambda_{1}^{2}:=y_{1}^{2}-4 y_{2}=\left.\left(x_{1}-x_{2}\right)^{2}\right|_{\begin{array}{l}
x_{1}+x_{2} \mapsto y_{1} \\
x_{1} x_{2} \mapsto y_{2}
\end{array}} .
$$

Applying $D_{(1)}$ (given in (4.27)) to $Q_{n}$ we find for the upper right block $Q_{n}^{(1)}=\mathcal{J}_{n}$

$$
\begin{aligned}
& D_{(1)}\left(\mathcal{J}_{n}\right)=Y_{*}\left(\left(x_{1}+\eta^{2} x_{2}\right)^{2} \mu_{2 n+1} \mu_{-2 n-1}+\left(x_{1}-\eta^{2} x_{2}\right)^{2} \pi_{2 n+1} \pi_{-2 n-1}\right) \\
& =\left(\begin{array}{cc}
y_{1}^{2} \mu_{2 n+3} \mu_{-2 n+1}+\lambda_{1}^{2} \pi_{2 n+3} \pi_{-2 n+1} & 2 y_{1} \lambda_{1}^{2} \mu_{2} \pi_{2} \\
2 y_{1} \mu_{2} \pi_{2} & y_{1}^{2} \mu_{2 n+3} \mu_{-2 n+1}+\lambda_{1}^{2} \pi_{2 n+3} \pi_{-2 n+1}
\end{array}\right) \\
& =\left(\mathcal{U}_{n}^{(0)}\right)^{-1} \cdot \mathcal{J}_{n(1)} \cdot \mathcal{U}_{n}^{(1)}
\end{aligned}
$$

with

$$
\mathcal{J}_{n(1)}=\left(\begin{array}{cc}
\mathcal{J}_{n-1} & 0 \\
y_{1} & \mathcal{J}_{n+1}
\end{array}\right) .
$$

In the last step we performed a similarity transformation to define a convenient form $Q_{n(1)}$ for the factorisation $D_{(1)}\left(Q_{n}\right)$,

$$
Q_{n(1)}=\mathcal{U}_{n} \cdot\left(D_{(1)}\left(Q_{n}\right)\right) \cdot\left(\mathcal{U}_{n}\right)^{-1},
$$


where the transformation $\mathcal{U}_{n}:={ }^{1} \mathcal{U}_{n}$ is defined by

$$
\begin{aligned}
{ }^{r} \mathcal{U}_{n}:= & \mathcal{U}_{\text {row } \times}\left(r+1 ; \frac{1}{\mu_{4}}\right) \cdot \mathcal{U}_{\text {row } \times}\left(r ; \frac{\pi_{2 n-1}}{\pi_{2 n+3}}\right) \cdot \mathcal{U}_{\text {col } \times}\left(r+1 ; \mu_{4} \frac{\pi_{-2 n-3}}{\pi_{-2 n+1}}\right) \\
& \cdot \mathcal{U}_{\text {col }}\left(r, r+1 ;-\frac{y_{1} \mu_{2 n+3}}{\pi_{2 n+3}}\right) \cdot \mathcal{U}_{\text {row }}\left(r+1, r ; \frac{y_{1} \mu_{2 n-1}}{\pi_{2 n-1}}\right) .
\end{aligned}
$$

Here, $\mathcal{U}_{\text {row } \times}(r ; \alpha)\left(\mathcal{U}_{\text {col } \times}(r ; \alpha)\right)$ has the effect of multiplying row $r$ (column $\left.r\right)$ of the upper right block $Q^{(1)}$ of a matrix factorisation with the constant $\alpha . \mathcal{U}_{\text {col }}(r, s ; \alpha)\left(\mathcal{U}_{\text {row }}(r, s ; \alpha)\right)$ has the effect on the block $Q^{(1)}$ of adding row $r$ (column $r$ ) multiplied by $\alpha$ to row $s$ (column $s)$. The precise conventions and explicit formulae for the similarity transformations are summarised in appendix A.

Let us now apply $D_{(1)}$ on $Q_{n(1)}$. The upper right block $Q_{n(1)}^{(1)}=\mathcal{J}_{n(1)}$ is given in (4.41), and it has the factors $\mathcal{J}_{n-1}$ and $\mathcal{J}_{n+1}$ on the diagonal, which will be mapped to $D_{(1)}\left(\mathcal{J}_{n \pm 1}\right)$. We then directly apply the similarity transformations to bring those to the form $\mathcal{J}_{n \pm 1(1)}$,

$$
\begin{aligned}
D_{(1)}\left(\mathcal{J}_{n(1)}\right) & =\left(\begin{array}{cc}
D_{(1)}\left(\mathcal{J}_{n-1}\right) & 0 \\
D_{(1)}\left(y_{1}\right) & D_{(1)}\left(\mathcal{J}_{n+1}\right)
\end{array}\right) \\
& =\left(\begin{array}{cc}
\mathcal{U}_{n-1}^{(0)} & 0 \\
0 & \mathcal{U}_{n+1}^{(0)}
\end{array}\right)^{-1} \cdot\left(\begin{array}{cc}
\frac{\mathcal{J}_{n-1(1)}}{D_{(1)}\left(y_{1}\right)_{n}} & 0 \\
\mathcal{J}_{n+1(1)}
\end{array}\right) \cdot\left(\begin{array}{cc}
\mathcal{U}_{n-1}^{(1)} & 0 \\
0 & \mathcal{U}_{n+1}^{(1)}
\end{array}\right)
\end{aligned}
$$

with

$$
\begin{aligned}
\widetilde{D_{(1)}\left(y_{1}\right)_{n}} & =\mathcal{U}_{n+1}^{(0)} \cdot D_{(1)}\left(y_{1}\right) \cdot\left(\mathcal{U}_{n-1}^{(1)}\right)^{-1} \\
& =\left(\begin{array}{cc}
\frac{y_{1} \pi_{2 n+3}}{\pi_{2 n+5}} & \frac{\mathcal{J}_{n} \mu_{2} \mu_{4}}{\pi_{2 n+5} \pi_{-2 n+3}} \\
\frac{1}{2 \pi_{2}} & \frac{y_{1} \pi_{-2 n+1}}{\pi_{-2 n+3}}
\end{array}\right) .
\end{aligned}
$$

The effect of the similarity transformation is summarised in the transformation

$$
\mathcal{U}_{n(1)}^{a}:={ }^{3} \mathcal{U}_{n+1} \cdot{ }^{1} \mathcal{U}_{n-1}
$$

where the left superscript $j$ on ${ }^{j} \mathcal{U}_{m}$ denotes the row and column where the corresponding $2 \times 2$-block $\mathcal{U}_{m}$ starts (in accordance with the definition in (4.43)).

We can perform further similarity transformations to bring $D_{(1)}\left(Q_{n(1)}\right)$ into a convenient form:

$$
\begin{aligned}
D_{(1)}\left(\mathcal{J}_{n(1)}\right) & =\left(\mathcal{U}_{n(1)}^{b(0)} \cdot \mathcal{U}_{n(1)}^{a(0)}\right)^{-1} \cdot\left(\begin{array}{cccc}
\mathcal{J}_{n-2} & 0 & 0 & 0 \\
y_{1} & \mathcal{J}_{n} & 0 & 0 \\
0 & -\frac{\mathcal{J}_{n}}{2 \pi_{2} \chi_{(n)}} & \mathcal{J}_{n} & 0 \\
\frac{1}{2 \pi_{2}} & 0 & y_{1} & \mathcal{J}_{n+2}
\end{array}\right) \cdot \mathcal{U}_{n(1)}^{b(1)} \cdot \mathcal{U}_{n(1)}^{a(1)} \\
& =\left(\mathcal{U}_{n(1)}^{c(0)} \cdot \mathcal{U}_{n(1)}^{b(0)} \cdot \mathcal{U}_{n(1)}^{a(0)}\right)^{-1} \cdot\left(\begin{array}{cccc}
\mathcal{J}_{n-2} & 0 & 0 & 0 \\
y_{1} & 0 & \mathcal{J}_{n} & 0 \\
0 & \mathcal{J}_{n} & 0 & 0 \\
\chi_{(n)} & 0 & y_{1} & \mathcal{J}_{n+2}
\end{array}\right) \cdot \mathcal{U}_{n(1)}^{c(1)} \cdot \mathcal{U}_{n(1)}^{b(1)} \cdot \mathcal{U}_{n(1)}^{a(1)}
\end{aligned}
$$


Here, the transformation $\mathcal{U}_{n(1)}^{b}$ is a simple row and column operation that deletes the entries $\propto y_{1}$

$$
\mathcal{U}_{n(1)}^{b}={ }^{2} \mathcal{U}_{n(1)}, \quad{ }^{r} \mathcal{U}_{n(1)}:=\mathcal{U}_{\text {col }}\left(r+1, r ;-\frac{\pi_{-2 n+1}}{\pi_{-2 n+3}}\right) \cdot \mathcal{U}_{\text {row }}\left(r, r+1 ;-\frac{\pi_{2 n+3}}{\pi_{2 n+5}}\right)
$$

while the transformation $\mathcal{U}_{n(1)}^{c}$ is defined as

$$
\begin{aligned}
\mathcal{U}_{n(1)}^{c}= & { }_{\tilde{c}}^{2} \mathcal{U}_{n(1)} \cdot{ }_{c}^{2} \mathcal{U}_{n(1)} \\
{ }_{c}^{r} \mathcal{U}_{n(1)}:= & \mathcal{U}_{\text {col }}\left(r, r+1 ; 2 \pi_{2} \chi_{(n)}\right) \cdot \mathcal{U}_{\text {row }}\left(r+1, r ; 2 \pi_{2} \chi_{(n)}\right) \\
{ }_{\tilde{c}}^{r} \mathcal{U}_{n(1)}:= & \mathcal{U}_{\text {row } \times}\left(r-1 ; \frac{1}{2 \pi_{2} \chi_{(n)}}\right) \cdot \mathcal{U}_{\text {col } \times}\left(r-1 ; 2 \pi_{2} \chi_{(n)}\right) \\
& \cdot \mathcal{U}_{\text {row } \times}\left(r ; \frac{1}{2 \pi_{2} \chi_{(n)}}\right) \cdot \mathcal{U}_{\text {col } \times}\left(r ;-2 \pi_{2} \chi_{(n)}\right) .
\end{aligned}
$$

For convenience we introduced the quantities

$$
\chi_{(p)}:=\frac{\pi_{-2 p+3} \pi_{2 p+5}}{4 \pi_{2}^{2} \pi_{-2 p+1} \pi_{2 p+3}} .
$$

Looking at (4.49) we see that the matrix factorisations can be split into the factorisation $Q_{n}$ and a new factorisation $Q_{n(2)}$ whose upper right block $Q_{n(2)}^{(1)}=\mathcal{J}_{n(2)}$ is

$$
\mathcal{J}_{n(2)}=\left(\begin{array}{ccc}
\mathcal{J}_{n-2} & 0 & 0 \\
y_{1} & \mathcal{J}_{n} & 0 \\
\chi_{(n)} & y_{1} & \mathcal{J}_{n+2}
\end{array}\right)
$$

In particular, we can identify the factorisation for the boundary state $|0,2\rangle$ as $Q_{|0,2\rangle}^{(1)}=\mathcal{J}_{0(2)}$.

One can now go on and apply $D_{(1)}$ again. We will show in appendix B that in this way one generates a family of factorisations $Q_{n(m)}$ with the property

$$
D_{(1)}\left(Q_{n(m)}\right) \cong Q_{n(m-1)} \oplus Q_{n(m+1)} .
$$

The upper right block $\mathcal{J}_{n(m)} \equiv Q_{n(m)}^{(1)}$ of $Q_{n(m)}$ is given by

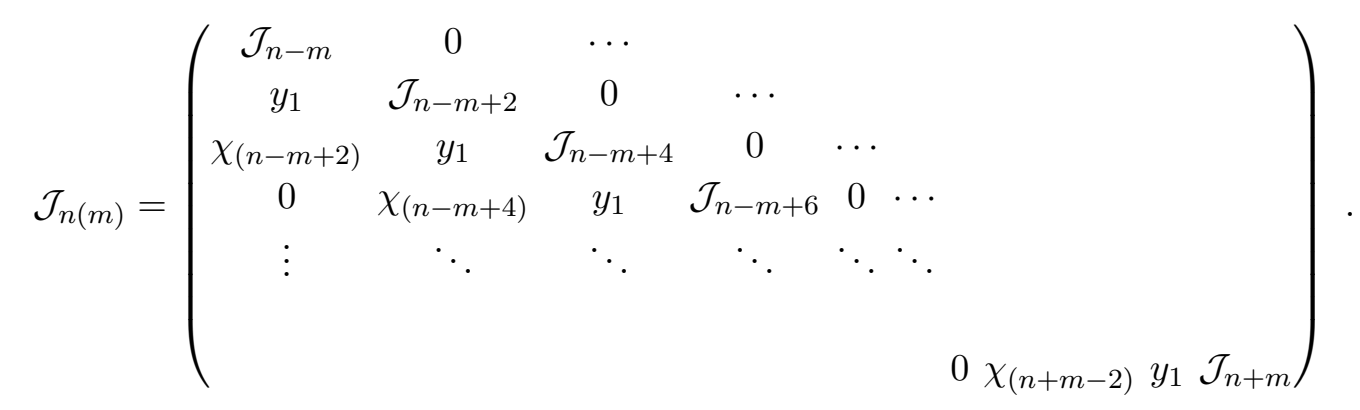

This formula applies for odd level $k$ for all $m \leq k+2$, whereas for even level $k$ it applies for $m+|n| \leq k / 2$. 
In particular one therefore has found matrix factorisations

$$
Q_{|0, \ell\rangle}=Q_{0(\ell)}
$$

for the rational boundary states $|0, \ell\rangle$ ! For odd $k$ this covers all boundary states of this type, whereas for even $k$ we have the restriction $\ell \leq k / 2$. Note however that (see (3.6))

$$
|L, \ell\rangle=\overline{|L, k+1-\ell\rangle},
$$

therefore boundary states with $\ell \geq k / 2+1$ can be related to boundary states with smaller label. The operation of taking the anti-boundary state corresponds in the matrix factorisation to an exchange of the blocks $Q^{(0)}$ and $Q^{(1)}$. Therefore we have found factorisations for all boundary states of the form $|0, \ell\rangle$.

As presented in appendix $\mathrm{E}$, it is possible to find a very compact alternative closed expression for the form of both the $\mathcal{J}_{n(m)} \equiv Q_{n(m)}^{(1)}$ block as well as of the $\mathcal{E}_{n(m)} \equiv Q_{n(m)}^{(0)}$ block of the matrix factorisations $Q_{n(m)}$. Referring to the appendix for the computational details, we would just like to mention here that the derivation is based on two major steps. In the first step, the structure of the $\mathcal{E}_{n(m)}$ blocks is inductively derived from the explicit formula (4.55) for $\mathcal{J}_{n(m)}$ via the basic equation

$$
Q_{n(m)}^{2}=W \cdot \mathbb{1} \quad \Leftrightarrow \quad \mathcal{E}_{n(m)}=W \cdot\left(\mathcal{J}_{n(m)}\right)^{-1} .
$$

The second step consists in applying a series of row and column operations on the $\mathcal{J}_{n(m)}$ block in order to "clear out" all rows and columns that intersect at a constant entry. According to $(4.55)$, this leaves a $2 \times 2$ non-trivial block $\widehat{\mathcal{J}}_{n(m)}$ in direct sum with $m-1$ trivial matrix factorisation blocks $\mathcal{J}_{\text {triv }}$. Upon closer inspection, the aforementioned similarity transformations induce operations on the $\mathcal{E}_{n(m)}$ block that leave the $2 \times 2$ subblock $\widehat{\mathcal{E}}_{n(m)}$ formed from the overlap of the last two lines and the first two columns of $\mathcal{E}_{n(m)}$ invariant. But since $\widehat{\mathcal{E}}_{n(m)}$ is thus just a subblock of $\mathcal{E}_{n(m)}$, in contrast to $\widehat{J}_{n(m)}$ we already know an explicit formula for $\widehat{\mathcal{E}}_{n(m)}$, and thus in turn also for $\widehat{\mathcal{J}}_{n(m)}$ :

$$
\begin{aligned}
& \widehat{\mathcal{E}}_{n(m)}=\left(\begin{array}{cc}
\Psi_{n-1(m-1)} & \Psi_{n(m-2)} \\
\Psi_{n(m)} & \Psi_{n+1(m-1)}
\end{array}\right) \\
& \widehat{\mathcal{J}}_{n(m)}=W \widehat{\mathcal{E}}_{n(m)}^{-1}=\frac{1}{W} \frac{\prod_{j=0}^{m} \mathcal{J}_{n-m+2 j}}{\prod_{j=1}^{m-1} \chi_{(n-m+2 j)}}\left(\begin{array}{cc}
\Psi_{n+1(m-1)} & -\Psi_{n(m-2)} \\
-\Psi_{n(m)} & \Psi_{n-1(m-1)}
\end{array}\right) .
\end{aligned}
$$

The explicit formula for the entries $\Psi_{n(m)}$ is given in (E.20) in appendix E.

\subsubsection{A closed formula for all rational matrix factorisations}

To obtain expressions for all rational matrix factorisations, we start from the factorisations $Q_{|L, 0\rangle}$ and apply $D_{(1)}$ successively to generate factorisations for the boundary states $|L, \ell\rangle$,

$$
D_{(1)}\left(Q_{|L, \ell\rangle}\right) \cong Q_{|L, \ell-1\rangle} \oplus Q_{|L, \ell+1\rangle} .
$$

The biggest computational problem is then the decomposition into the elementary factorisations on the right hand side. This was already tedious for $L=0$ where we started from 
a degree 2 polynomial $\mathcal{J}_{0}$, so a priori, it appears hopeless to find a closed formula for the factorisations $Q_{|L, \ell\rangle}$ with $\ell>1$, where the starting polynomial

$$
\mathcal{J}_{|L, 0\rangle}=\prod_{i=0}^{L} \mathcal{J}_{i}
$$

is of degree $2(L+1)$. We may however rewrite the higher polynomial factorisations as cones of the elementary polynomial factorisations (see e.g. [13]), such that (we will again only write the upper right block of the matrix factorisations)

$$
\mathcal{J}_{|L, 0\rangle}=\prod_{i=0}^{L} \mathcal{J}_{i} \cong\left(\begin{array}{cccccccc}
\mathcal{J}_{0} & 0 & \ldots & & & & \\
1 & \mathcal{J}_{1} & 0 & \ldots & & & \\
0 & 1 & \mathcal{J}_{2} & 0 & \ldots & & \\
\vdots & \ddots & \ddots & \ddots & & & \\
& & & 0 & 1 & \mathcal{J}_{L-1} & 0 \\
& & & & 0 & 1 & \mathcal{J}_{L}
\end{array}\right)
$$

Each of the diagonal entries of the cone is simply a polynomial factor $\mathcal{J}_{n}$ of degree 2 . When we now apply $D_{(1)}$ successively, we can in principle use our results of the previous subsection to obtain factorisations with blocks $\mathcal{J}_{n(m)}$ on the diagonal.

The difficulty in this approach is that the similarity transformations that are used to arrive at the blocks $\mathcal{J}_{n(m)}$ will also affect the morphisms. When we apply $D_{(1)}$ in the first step its action on the morphisms 1 is trivial,

$$
D_{(1)}(1)=\mathbb{1}
$$

but the similarity transformations will produce non-trivial entries. As an example consider the matrix

$$
\mathcal{J}_{p, q}=\left(\begin{array}{cc}
\mathcal{J}_{p} & 0 \\
1 & \mathcal{J}_{q}
\end{array}\right)
$$

When we apply $D_{(1)}$ on it and transform the diagonal blocks $D_{(1)}\left(\mathcal{J}_{n}\right)$ into the form $\mathcal{J}_{n(1)}$ (see (4.41)) via the similarity transformations $\mathcal{U}_{n}$ (see (4.42)), we obtain

$$
D_{(1)}\left(\mathcal{J}_{p, q}\right) \cong\left(\begin{array}{cccc}
\mathcal{J}_{p-1} & 0 & 0 & 0 \\
y_{1} & \mathcal{J}_{p+1} & 0 & 0 \\
\frac{\pi_{2 q-1}}{\pi_{2 q+3}} & \frac{y_{1} \mu_{4} \mu_{2 q-2 p-4}}{\pi_{2 q+3} \pi_{-2 p+1}} & \mathcal{J}_{q-1} & 0 \\
0 & \frac{\pi_{-2 p-3}}{\pi_{-2 p+1}} & y_{1} & \mathcal{J}_{q+1}
\end{array}\right)
$$

While now the diagonal blocks are in the right form to apply our inductive mechanism for finding the result of applying $D_{(1)}$ to them, we observe that since now the morphisms between the $Q_{n(1)}$-type blocks have an entry of polynomial degree $>0\left(\propto y_{1}\right)$, each time we apply $D_{(1)}$ we will generate consecutively higher degree polynomial morphism entries, thus leading to an extremely complex morphism structure.

We have instead to look for an alternative standard form for the $\mathcal{J}_{n(m)}$ that is obtained by using similarity transformations that leave the morphisms (the identity matrices) unchanged. A prototype of such a transformation is one that 
- does not depend on $n$, and

- has identical diagonal blocks, $\mathcal{U}^{(0)}=\mathcal{U}^{(1)}$.

Then the morphism entries are unaffected,

$$
\mathcal{U}^{(0)} \cdot \mathbb{1} \cdot\left(\mathcal{U}^{(1)}\right)^{-1}=\mathbb{1}
$$

Our strategy, however, was to allow for all similarity transformations a priori, and then make sure at the end that all morphisms are again identity matrices. We conjecture that it is enough to use transformations with the two properties described above, but it is not guaranteed from our analysis.

To describe the alternative standard form we found in this way, we have to introduce some notation. First let us define a generalisation of the functor $D_{(1)}$,

$$
\tilde{D}_{0, m}:=Y_{*} \circ \sigma_{\eta^{2 m}}^{*} \circ Y^{*},
$$

i.e. we first express the variables $y_{i}$ through the $x_{j}$, then map $x_{2} \mapsto \eta^{2 m} x_{2}$, and then apply the functor $Y_{*}$ to again obtain a matrix in the variables $y_{i}$. For $m=1$ we have $D_{(1)}=\tilde{D}_{0,1}$ (see (4.27)). The action of $\tilde{D}_{0, m}$ on an elementary polynomial factor $\mathcal{J}_{n}$ is given by ${ }^{4}$

$$
\begin{aligned}
\tilde{D}_{0, m}\left(\mathcal{J}_{n}\right) & =\left(y_{1}^{2} \mu_{2 m+2 n+1} \mu_{2 m-2 n-1}+\lambda_{1}^{2} \pi_{2 m+2 n+1} \pi_{2 m-2 n-1}\right) \cdot \mathbb{1}+2 y_{1} \lambda_{1} \mu_{2 m} \pi_{2 m} \Lambda, \\
\Lambda & :=\left(\begin{array}{cc}
0 & \lambda_{1} \\
\frac{1}{\lambda_{1}} & 0
\end{array}\right) .
\end{aligned}
$$

It is worthwhile to note the origin of the two elementary matrices $\mathbb{1}$ and $\Lambda$ in this formula, which is simply the application of the "symmetrisation fusion functor" $Y_{*}$ onto $y_{1} \equiv x_{1}+x_{2}$ and $\lambda_{1} \equiv x_{1}-x_{2}$ (i.e. to $y_{1}$ upon embedding into the polynomial ring $\mathbb{C}\left[x_{1}, x_{2}\right]$, and to $\lambda_{1}$ considered as an element of $\left.\mathbb{C}\left[x_{1}, x_{2}\right]\right)$ :

$$
Y_{*}\left(y_{1}\right)=y_{1} \mathbb{1}, \quad Y_{*}\left(\lambda_{1}\right)=\lambda_{1} \Lambda .
$$

The crucial feature of (4.68) is the fact that the off-diagonal entries of $\tilde{D}_{0, m}\left(\mathcal{J}_{n}\right)$ do not depend on $n$, i.e. on the label of the elementary polynomial $\mathcal{J}_{n}$.

For later convenience, we will also define the symbol $\tilde{\tilde{D}}_{0, m}\left(\mathcal{J}_{n}\right)$ to denote the following form for $\tilde{D}_{0, m}\left(\mathcal{J}_{n}\right)$, which is obtained via a similarity transformation that rescales the off-diagonal entries: ${ }^{5}$

$$
\begin{aligned}
& \tilde{\tilde{D}}_{0, m}\left(\mathcal{J}_{n}\right):=\tilde{\tilde{\mathcal{U}}}_{m, n}^{(0)} \cdot\left(\tilde{D}_{0, m}\left(\mathcal{J}_{n}\right)\right) \cdot \tilde{\tilde{\mathcal{U}}}_{m, n}^{(1)^{-1}} \\
& =\left(y_{1}^{2} \mu_{2 m+2 n+1} \mu_{2 m-2 n-1}+\lambda_{1}^{2} \pi_{2 m+2 n+1} \pi_{2 m-2 n-1}\right) \mathbb{1} \\
& +\left(\begin{array}{cc}
0 & 4 \mu_{2 m}^{2} \pi_{2 m}^{2} y_{1} \lambda_{1}^{2} \\
y_{1} & 0
\end{array}\right) \\
& \tilde{\tilde{\mathcal{U}}}_{m, n}:=\mathcal{U}_{\text {col } \times}\left(2 ; 2 \mu_{2 m} \pi_{2 m}\right) \cdot \mathcal{U}_{\text {row } \times}\left(2 ; \frac{1}{2 \mu_{2 m} \pi_{2 m}}\right) \text {. }
\end{aligned}
$$

\footnotetext{
${ }^{4}$ Note that the entries of $\Lambda$ as well as $\lambda_{1}$ are not elements of the polynomial ring $\mathbb{C}\left[y_{1}, y_{2}\right]$, but that the combination $\lambda_{1} \Lambda$ that appears in the formulae has entries that can be written as polynomials in $y_{1}, y_{2}$.

${ }^{5}$ This similarity transformation is independent of $n$ and has identical diagonal blocks, so it satisfies the two criteria specified above.
} 
We want to take this as our new standard form for $\mathcal{J}_{n(1)}$, so we define

$$
\tilde{\mathcal{J}}_{n(1)}:=\tilde{\tilde{D}}_{0,1}\left(\mathcal{J}_{n}\right)
$$

We can immediately conclude that

$$
D_{(1)}\left(\mathcal{J}_{|L, 0\rangle}\right) \cong\left(\begin{array}{ccccccc}
\tilde{\mathcal{J}}_{0(1)} & 0 & \ldots & & & \\
\mathbb{1} & \tilde{\mathcal{J}}_{1(1)} & 0 & \ldots & & & \\
0 & \mathbb{1} & \tilde{\mathcal{J}}_{2(1)} & 0 & \cdots & & \\
\vdots & \ddots & \ddots & \ddots & & & \\
& & & 0 & \mathbb{1} & \tilde{\mathcal{J}}_{L-1(1)} & 0 \\
& & & & 0 & \mathbb{1} & \tilde{\mathcal{J}}_{L(1)}
\end{array}\right)
$$

Now we have to look for similar expressions for $\mathcal{J}_{n(m)}$ for $m \geq 2$. A tedious computation (some ideas of which are presented in appendix C) leads to the following claim: we have found an alternative form of $Q_{n(m)}$ that we call $\tilde{Q}_{n(m)}$ (related by a similarity transformation) and that satisfies the following property: denote by $C\left(p_{1}, \ldots, p_{r} ; m\right)$ the cone whose upper right block is given by

$$
C\left(p_{1}, \ldots, p_{r} ; m\right)^{(1)}=\left(\begin{array}{ccccccccc}
\tilde{\mathcal{J}}_{p_{1}(m)} & 0 & \ldots & & & & \\
\mathbb{1} & \tilde{\mathcal{J}}_{p_{2}(m)} & 0 & \ldots & & & \\
0 & \mathbb{1} & \tilde{\mathcal{J}}_{p_{3}(m)} & 0 & \ldots & & \\
\vdots & \ddots & \ddots & \ddots & & & \\
& & & 0 & \mathbb{1} & \tilde{\mathcal{J}}_{p_{r-1}(m)} & 0 \\
& & & & 0 & \mathbb{1} & \tilde{\mathcal{J}}_{p_{r}(m)}
\end{array}\right)
$$

where as usual $\tilde{\mathcal{J}}_{n(m)}$ is the upper right block of $\tilde{Q}_{n(m)}$. Then

$$
D_{(1)}\left(C\left(p_{1}, \ldots, p_{r} ; m\right)\right) \cong C\left(p_{1}, \ldots, p_{r} ; m-1\right) \oplus C\left(p_{1}, \ldots, p_{r} ; m+1\right)
$$

for generic $p_{1}, \ldots, p_{r}$. The alternative standard form $\tilde{\mathcal{J}}_{n(m)}$ is given by

for even $m$ :

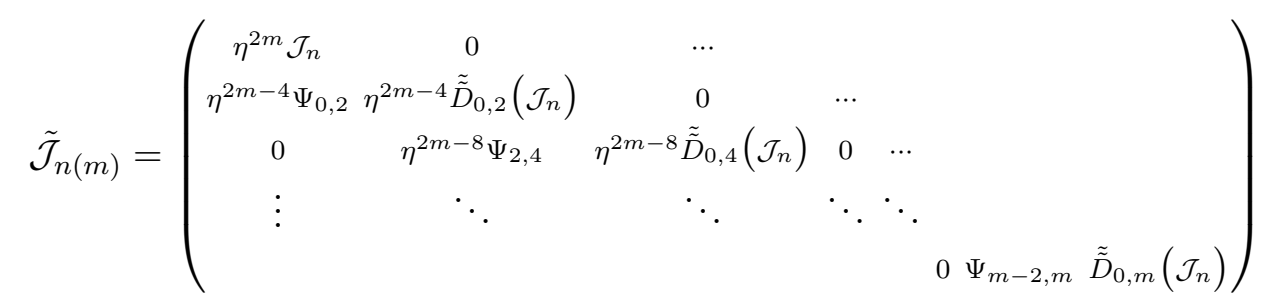

for odd $m$ :

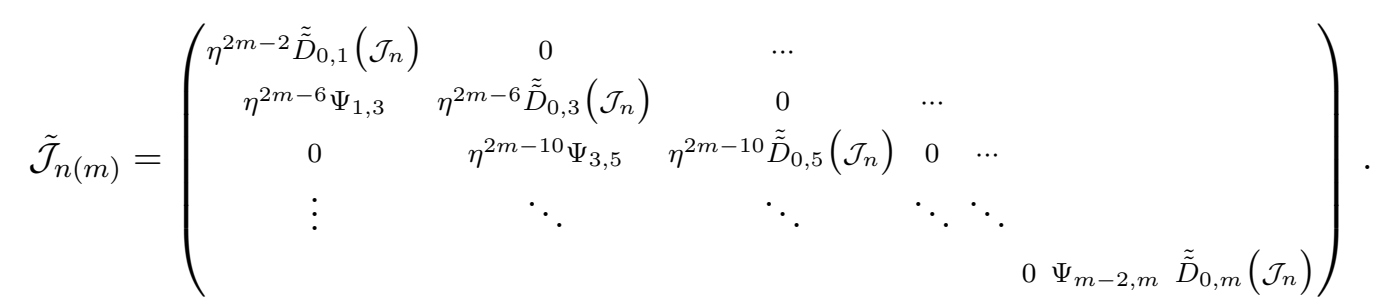


This formula is obtained by an extrapolation of the pattern one observes for small values $m$. We expect it to be correct for $m \leq k+2$ if $k$ is odd, whereas for $k$ even we can from our derivation only conclude that it should be valid for $m+\left|p_{i}\right| \leq k / 2$ (see the discussion in appendix D). On the other hand, we have reasons to believe that it is valid for a larger set of values for $m$ : we conjectured above that the decomposition in (4.74) can also be done purely by using similarity transformations that satisfy the two properties formulated above, i.e. by blockwise transformations independent of the label $p_{i}$. If this is true, then also the constraint should not depend on the label $p_{i}$ and we could conclude that the formula is valid for all $m \leq k / 2$.

We can then finally write down a matrix factorisation for a general rational boundary state $|L, \ell\rangle$ in the form

$$
\mathcal{J}_{|L, \ell\rangle} \cong\left(\begin{array}{ccccccc}
\tilde{\mathcal{J}}_{0(\ell)} & 0 & \ldots & & & & \\
\mathbb{1} & \tilde{\mathcal{J}}_{1(\ell)} & 0 & \ldots & & & \\
0 & \mathbb{1} & \tilde{\mathcal{J}}_{2(\ell)} & 0 & \ldots & & \\
\vdots & \ddots & \ddots & \ddots & & & \\
& & & 0 & \mathbb{1} & \tilde{\mathcal{J}}_{L-1(\ell)} & 0 \\
& & & & 0 & \mathbb{1} & \tilde{\mathcal{J}}_{L(\ell)}
\end{array}\right)
$$

For odd $k$ this formula should hold for all $L$ and $\ell$, whereas for even $k$ we have constraints. From the discussion above we conclude that it should be valid at least for $L+\ell \leq k / 2$, but if our conjecture on the similarity transformation is correct, it should hold for all $\ell \leq k / 2$. If this is true then using the identification

$$
|L, \ell\rangle=\overline{|L, k+1-\ell\rangle}
$$

one can get a factorisation for every rational boundary state also for even $k$.

Up to this issue of the constraints due to the level $k$, we have formulated a complete dictionary between matrix factorisations and rational boundary states for the KazamaSuzuki model of type $\mathrm{SU}(3)_{k} / \mathrm{U}(2)$.

\subsection{Effects of finite levels}

For a finite level $k$ there are only finitely many rational boundary states, so that if we continue to apply $D_{(1)}$ we should see dependencies between the factorisations that arise due to the identity

$$
\eta^{k+3}=-1
$$

Checking the dependencies is then another test that we identified the correct matrix factorisation.

When we successively determine factorisations by applying the fusion functor $D_{(1)}$ on 
factorisations $Q_{|L, \ell\rangle}$ we expect our first interesting effect for the special value $\ell=\left\lfloor\frac{k+1}{2}\right\rfloor$ :

$$
\begin{aligned}
& k \in 2 \mathbb{Z} \\
& D_{(1)}\left(Q_{\left|L, \frac{k}{2}\right\rangle}\right) \cong Q_{\left|L, \frac{k}{2}-1\right\rangle} \oplus \underbrace{Q_{\left|L, \frac{k}{2}+1\right\rangle}}_{=Q \overline{\left|L, \frac{k}{2}\right\rangle}} \\
& k \in 2 \mathbb{Z}+1 \\
& D_{(1)}\left(Q_{\left|L, \frac{k+1}{2}\right\rangle}\right) \cong Q_{\left|L, \frac{k-1}{2}\right\rangle} \oplus \underbrace{}_{=Q \frac{Q_{\left|L, \frac{k-1}{2}\right\rangle}}{Q_{\left|L, \frac{k+3}{2}\right\rangle}}} .
\end{aligned}
$$

We notice a crucial difference in the cases $k$ odd and $k$ even, respectively: ${ }^{6}$ for $k$ even, there exists one special irreducible factor of the superpotential $W_{2 ; k}^{y}$ as defined in (4.1), namely the factor $\mathcal{J}_{\frac{k}{2}}=y_{1}$. We will thus have to discuss the two cases separately.

For the case $k$ odd, all the irreducible factors $\mathcal{J}_{i}$ of the superpotential $W_{2 ; k}^{y}$ are of the generic form (4.36), so the only effect of the special label $\ell=\frac{k+1}{2}$ consists in a number of identifications. For concreteness, consider the case of the rational matrix factorisations $Q_{|0, \ell\rangle}$, for which we found earlier the formula (see (4.55))

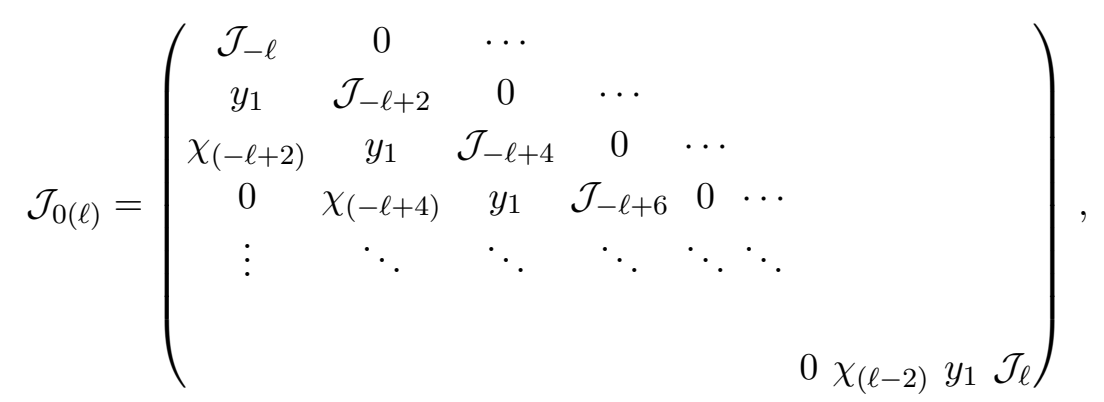

with $\chi_{(p)}$ defined in (4.52). Using the obvious identification of labels

$$
\mathcal{J}_{-n}=y_{1}^{2} \mu_{-2 n+1} \mu_{2 n-1}+\lambda_{1}^{2} \pi_{-2 n+1} \pi_{2 n-1}=\mathcal{J}_{n-1},
$$

we observe that the negative labels in (4.81) are mapped to positive labels in such a way that for $\ell=\frac{k+1}{2}$ the list of diagonal entries of $\mathcal{J}_{|0, \ell\rangle}$ exhausts the list of all irreducible factors (which are labelled $\mathcal{J}_{0}, \mathcal{J}_{1}, \ldots, \mathcal{J}_{\frac{k+1}{2}}$ for $k$ odd). It may be checked that (unlike in the case of $k$ even, which will be discussed below) no special relations play a role when applying $D_{(1)}$ to $Q_{\left|0, \frac{k+1}{2}\right\rangle}$, i.e. we obtain our usual result

$$
D_{(1)}\left(Q_{\left|0, \frac{k+1}{2}\right\rangle}\right) \cong Q_{\left|0, \frac{k-1}{2}\right\rangle} \oplus Q_{\left|0, \frac{k+3}{2}\right\rangle} .
$$

The only structural speciality in $Q_{\left|0, \frac{k+3}{2}\right\rangle}$ stems from the fact that

$$
\mathcal{J}_{-\frac{k+3}{2}}=\mathcal{J}_{\frac{k+1}{2}}=\mathcal{J}_{\frac{k+3}{2}},
$$

which may be checked by inspecting (4.36). In addition, the relation

$$
\chi_{(-m)}=\chi_{(m-1)},
$$

\footnotetext{
${ }^{6}$ See also figure 1 of [13] for illustration.
} 
which follows immediately from the definition (4.52) of $\chi_{(m)}$, may be employed to convert every constant $\chi_{(m)}$ with negative label into one with positive label. Additional arguments for proving the second part of the claim, i.e. that $Q_{\left|L, \frac{k+3}{2}\right\rangle} \cong Q_{\left|L, \frac{k-1}{2}\right\rangle}$, are introduced below when we discuss the case of even $k$, but we refrain from carrying out the explicit computations for brevity, since they are entirely analogous to those necessary in the more interesting case of $k$ even.

For the case $k$ even, we encounter the problem that the formula $(4.81)$ for $\mathcal{J}_{0(\ell)}$ is only valid for $\ell \leq \frac{k}{2}$. Therefore when we want to check (4.79), we cannot directly use the formula (4.81) for $Q_{\left|0, \frac{k}{2}+1\right\rangle}$. The problem occurs when $D_{(1)}$ hits the polynomial factor $\mathcal{J}_{p}$ with highest $p(p=k / 2)$ on the diagonal of $\mathcal{J}_{0(k / 2)}$. We then have (see (4.68))

$$
\begin{aligned}
D_{(1)}\left(\mathcal{J}_{k / 2}\right) & =\left(y_{1}^{2} \mu_{k+3} \mu_{-k+1}+\lambda_{1}^{2} \pi_{k+3} \pi_{-k+1}\right) \cdot \mathbb{1}+y_{1} \lambda_{1} \mu_{4} \Lambda \\
& =\pi_{-2 k-2} y_{1}^{2} \mathbb{1}+\mu_{-2 k-2} y_{1} \lambda_{1} \Lambda .
\end{aligned}
$$

It is now a straightforward computation to demonstrate that via the similarity transformations

$$
\begin{aligned}
\hat{\mathcal{U}}_{(k)}:= & \mathcal{U}_{\text {col } \times}\left(1 ; \frac{1}{\mu_{-2 k-2}}\right) \cdot \mathcal{U}_{\text {row } \times}\left(1 ; \mu_{2 k+2}\right) \cdot \\
& \cdot \mathcal{U}_{\text {col }}\left(1,2, \frac{y_{1} \pi_{2 k+2}}{\mu_{2 k+2}}\right) \cdot \mathcal{U}_{\text {row }}\left(1,2, \frac{y_{1} \pi_{2 k+2}}{\mu_{2 k+2}}\right)
\end{aligned}
$$

we may realise the isomorphism

$$
\begin{aligned}
D_{(1)}\left(\mathcal{J}_{k / 2}\right) & \cong\left(\begin{array}{cc}
0 & y_{1}\left(\pi_{2 k+2} \pi_{-2 k-2} y_{1}^{2}+\mu_{2 k+2} \mu_{-2 k-2} \lambda_{1}^{2}\right) \\
y_{1} & 0
\end{array}\right) \\
& =\left(\begin{array}{cc}
0 & y_{1} \mathcal{J}_{\frac{k}{2}-1} \\
y_{1} & 0
\end{array}\right) .
\end{aligned}
$$

Here, we have made use of the fact that

$$
\pi_{p}=\mu_{p+k+3} .
$$

We are now in the position to determine $\mathcal{J}_{0\left(\frac{k}{2}+1\right)}$ that occurs in the relation (4.79) for $L=0$

$$
k \in 2 \mathbb{Z}: \quad D_{(1)} \circ Q_{\left|0, \frac{k}{2}\right\rangle} \cong Q_{\left|0, \frac{k}{2}-1\right\rangle} \oplus Q_{\left|0, \frac{k}{2}+1\right\rangle} \stackrel{!}{\cong} Q_{\left|0, \frac{k}{2}-1\right\rangle} \oplus Q_{\left|0, \frac{k}{2}\right\rangle} .
$$

We start from the explicit formula (4.81) for the factorisation $Q_{|0, \ell\rangle}$, which reads using the relations (4.82) and (4.85):

$$
\mathcal{J}_{0(\ell)}=\left(\begin{array}{cccccccccc}
\mathcal{J}_{\ell-1} & 0 & \ldots & & & & & \\
y_{1} & \mathcal{J}_{\ell-3} & 0 & \ldots & & & & \\
\chi_{(\ell-3)} & y_{1} & \mathcal{J}_{\ell-5} & 0 & \ldots & & & \\
0 & \chi_{(\ell-5)} & y_{1} & \mathcal{J}_{\ell-7} & 0 & \ldots & & \\
\vdots & \ddots & \ddots & \ddots & \ddots & \ddots & & \\
& & & & & & & \\
& & & & & & 0 & \chi_{(\ell-2)} & y_{1} & \mathcal{J}_{\ell}
\end{array}\right)
$$


Here, the entries on the diagonal run from $\mathcal{J}_{\ell-1}$ to $\mathcal{J}_{\ell-m^{*}}$ in steps of two, where

$$
m^{*}:= \begin{cases}\ell & \ell \text { odd } \\ \ell-1 & \ell \text { even. }\end{cases}
$$

Then, if $m^{*}=\ell$, the next diagonal entries after $\mathcal{J}_{0}$ read $\mathcal{J}_{1}, \mathcal{J}_{3}, \ldots$ Otherwise, we have that $\mathcal{J}_{\ell-m^{*}}=\mathcal{J}_{1}$, after which the next entries read $\mathcal{J}_{0}, \mathcal{J}_{2}, \mathcal{J}_{4}, \ldots$

At $k=2$ we obtain

$$
\mathcal{J}_{|0,1\rangle}=\left(\begin{array}{cc}
\mathcal{J}_{-1} & 0 \\
y_{1} & \mathcal{J}_{1}
\end{array}\right)
$$

and we immediately compute

$$
\begin{aligned}
& \left.D_{(1)}\left(\mathcal{J}_{|0,1\rangle}\right)\right|_{k=2}=\left(\begin{array}{c|c}
D_{(1)}\left(\mathcal{J}_{-1}\right) & 0 \\
\hline D_{(1)}\left[y_{1}\right] & D_{(1)}\left(\mathcal{J}_{1}\right)
\end{array}\right) \\
& \stackrel{(4.86)}{=}\left(\begin{array}{cc|cc}
\left(y_{1}^{2} \mu_{1} \mu_{3}+\lambda_{1}^{2} \pi_{1} \pi_{3}\right) & y_{1} \lambda_{1}^{2} \mu_{4} & 0 & 0 \\
y_{1} \mu_{4} & \left(y_{1}^{2} \mu_{1} \mu_{3}+\lambda_{1}^{2} \pi_{1} \pi_{3}\right) & 0 & 0 \\
\hline y_{1} \pi_{2} & \lambda_{1}^{2} \mu_{2} & y_{1}^{2} \mu_{-1} & y_{1} \lambda_{1}^{2} \pi_{-1} \\
\mu_{2} & y_{1} \pi_{2} & y_{1} \pi_{-1} & y_{1}^{2} \mu_{-1}
\end{array}\right) \text {. }
\end{aligned}
$$

Applying the transformation $\hat{\mathcal{U}}_{k}$ (see (4.87)) to the lower right block, and the standard transformation ${ }^{1} \mathcal{U}_{-1}$ (given in (4.43)) to the upper left block, we obtain the intermediate result

$$
\begin{aligned}
\left.D_{(1)}\left(\mathcal{J}_{|0,1\rangle}\right)\right|_{k=2} & \cong\left(\begin{array}{cc|cc}
\mathcal{J}_{1} & 0 & 0 & 0 \\
y_{1} & \mathcal{J}_{0} & 0 & 0 \\
\hline y_{1} \pi_{3} & \mathcal{J}_{0} \frac{\mu_{1} \pi_{1}}{\mu_{-1}} & 0 & y_{1} \mathcal{J}_{0} \\
2 \mu_{1} \pi_{1} & y_{1} \frac{\pi_{1} \pi_{-1}}{\pi_{3}} & y_{1} & 0
\end{array}\right) \\
& \cong\left(\begin{array}{cc|cc}
\mathcal{J}_{1} & 0 & 0 & 0 \\
y_{1} & \mathcal{J}_{0} & 0 & 0 \\
\hline 0 & \mathcal{J}_{0}\left(\frac{\mu_{1} \pi_{1}}{\mu_{-1}}-\pi_{3}\right) & 0 & y_{1} \mathcal{J}_{0} \\
2 \mu_{1} \pi_{1} & 0 & y_{1} & 0
\end{array}\right) \\
& \cong\left(\begin{array}{cc|cc}
\mathcal{J}_{1} & 0 & 0 & 0 \\
y_{1} & 0 & 0 & y_{1} \mathcal{J}_{0} \\
\hline 0 & \mathcal{J}_{0} & 0 & 0 \\
1 & 0 & y_{1} & 0
\end{array}\right) .
\end{aligned}
$$

It is then immediately obvious that this result can be transformed into the form

$$
\left.D_{(1)}\left(\mathcal{J}_{|0,1\rangle}\right)\right|_{k=2} \cong\left(\begin{array}{cc|cc}
0 & 0 & y_{1} \mathcal{J}_{1} & 0 \\
0 & 0 & -y_{1}^{2} & y_{1} \mathcal{J}_{0} \\
\hline 0 & \mathcal{J}_{0} & 0 & 0 \\
1 & 0 & 0 & 0
\end{array}\right)=\mathcal{J}_{\text {triv }} \oplus \mathcal{J}_{|0,0\rangle} \oplus \mathcal{J}_{\overline{|0,1\rangle}}
$$

which provides an explicit check of the relation (4.90) for $k=2$. 
Using the same tools as introduced in the computation of the explicit formula for the rational factorisations $Q_{|0, \ell\rangle}$, we can compute for example the next two cases for the level $k$ :

$$
\begin{aligned}
\left.D_{(1)}\left(\mathcal{J}_{|0,2\rangle}\right)\right|_{k=4} \cong \mathcal{J}_{|0,1\rangle} \oplus & \left(\begin{array}{cc|cc}
\mathcal{J}_{-3} & 0 & 0 & 0 \\
y_{1} & \mathcal{J}_{-1} & 0 & 0 \\
\chi_{(-1)} & y_{1} & 0 & y_{1} \\
\hline 0 & 1 & y_{1} & 0
\end{array}\right) \\
\left.D_{(1)}\left(\mathcal{J}_{|0,3\rangle}\right)\right|_{k=6} \cong \mathcal{J}_{|0,2\rangle} \oplus & \left(\begin{array}{ccc|cc}
\mathcal{J}_{-4} & 0 & 0 & 0 & 0 \\
y_{1} & \mathcal{J}_{-2} & 0 & 0 & 0 \\
\chi_{(-2)} & y_{1} & \mathcal{J}_{0} & 0 & 0 \\
0 & \chi_{(0)} & y_{1} & 0 & y_{1} \mathcal{J}_{2} \\
\hline 0 & 0 & 1 & y_{1} & 0
\end{array}\right) .
\end{aligned}
$$

We observe that the largest part of the factorisation $Q_{\left|0, \frac{k}{2}+1\right\rangle}$ is of the form of an ordinary factorisation $Q_{|0, \ell\rangle}$. From the first three even $k$ examples, we conjecture the formula

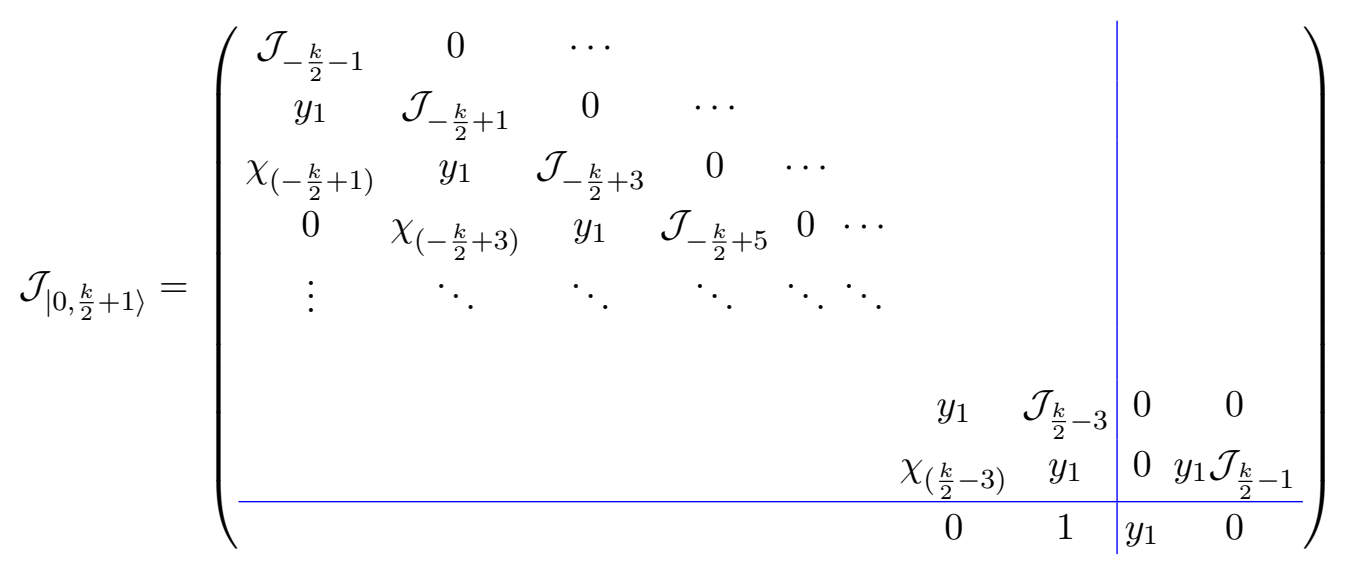

It remains to check that

$$
Q_{\left|0, \frac{k}{2}+1\right\rangle} \cong Q_{\overline{\left|0, \frac{k}{2}\right\rangle}},
$$

or in other words that the upper right block $\mathcal{J}_{\left|0, \frac{k}{2}+1\right\rangle}$ of $Q_{\left|0, \frac{k}{2}+1\right\rangle}$ can be transformed to the lower left block $\mathcal{E}_{\left|0, \frac{k}{2}\right\rangle}$ of the factorisation $Q_{\left|0, \frac{k}{2}+1\right\rangle}$ by elementary row and column operations.

Let us consider the example $k=4$. Note that $\mathcal{J}_{|0,3\rangle}$ has two constant entries that we can use to remove all other entries in their rows and columns, and we obtain

$$
\begin{aligned}
\left.\mathcal{J}_{|0,3\rangle}\right|_{k=4} & =\left(\begin{array}{cc|cc}
\mathcal{J}_{2} & 0 & 0 & 0 \\
y_{1} & \mathcal{J}_{0} & 0 & 0 \\
\chi_{(0)} & y_{1} & 0 & y_{1} \mathcal{J}_{1} \\
\hline 0 & 1 & y_{1} & 0
\end{array}\right) \\
& \cong\left(\begin{array}{cc|cc}
0 & 0 & -y_{1}^{2} \mathcal{J}_{2} & y_{1} \mathcal{J}_{2} \mathcal{J}_{1} \\
0 & 0 & y_{1}\left(y_{1}^{2}-\mathcal{J}_{0} \chi_{(0)}\right) & -y_{1}^{2} \mathcal{J}_{1} \\
\hline 0 & 1 & 0 & 0 \\
1 & 0 & 0 & 0
\end{array}\right) .
\end{aligned}
$$


The upper right block of this last form coincides with $\widehat{\mathcal{E}}_{0(2)}$ given in (E.35), which therefore proves the relation (4.98) in this case. Similarly we have verified (4.98) explicitly also for $k=6$. We take this as another convincing check that we identified the correct matrix factorisations.

\section{Conclusion and outlook}

In this article we have constructed matrix factorisations for rational boundary conditions in the $\mathrm{SU}(3) / \mathrm{U}(2)$ Kazama-Suzuki models. For the construction it was essential to identify the rational defect $D_{[(0,0), 0 ; 1,3]}$ in the Landau-Ginzburg description. Fusing this defect to boundary conditions $|L, 0\rangle$, one can generate all boundary conditions $|L, \ell\rangle$. Therefore by fusing the defect in the Landau-Ginzburg description to the matrix factorisations describing $|L, 0\rangle$, we can obtain all others.

To actually construct these matrix factorisations, it is important to have an efficient way of computing the fusion. We found an operator-like description for the fusion of the defect factorisation corresponding to $D_{[(0,0), 0 ; 1,3]}$ to another factorisation (see $(4.27)$ ), which is given by a specific operation on each entry of the factorisation. In this way we worked out the matrix factorisations for all rational boundary conditions $|L, \ell\rangle$, and hence have obtained a conjecture for a complete dictionary between the Landau-Ginzburg formulation and the rational conformal field theory description of the $\mathrm{SU}(3) / \mathrm{U}(2)$ Kazama-Suzuki models. More precisely, we proved our formula (4.55) (and the alternative compact version (4.59)) for the matrix factorisations $Q_{|0, \ell\rangle}$ explicitly, while for the $Q_{|L, \ell\rangle}$ factorisations with $L>0$ we have extrapolated the pattern we have observed for small values of $L$ to derive the conjecture for their explicit form (see (4.76)). Additional support for our conjecture comes from a detailed discussion of the effects of finite levels $k$, which are consistent with the expectations from the conformal field theory side of the dictionary. We will report in [48] a number of further structural arguments in favor of our conjecture.

Operator-like defects turn out to be very important for explicit computations. The process of fusing a defect factorisation of $W(x)-W(\tilde{x})$ to some matrix factorisation of $W(\tilde{x})$ is described by the tensor product, resulting in a factorisation of $W(x)$. This tensor product still contains the variables $\tilde{x}$. To eliminate these auxiliary variables can be a complicated task, though there are some strategies and algorithms known how this can be done $[21,49]$. For operator-like defects such as $D_{(1)}$, this step does not have to be performed - the process of fusing it to another factorisation is implemented by a functor that acts on the category of modules over a polynomial ring. ${ }^{7}$ In this functorial language one can also realise the morphisms of operator-like defects as morphisms between the corresponding functors, and in this way one can even define cones of functors in certain situations. This will be presented in [50].

For the $\mathrm{SU}(3) / \mathrm{U}(2)$ Kazama-Suzuki models it turns out that all rational B-type defects can be realised as operator-like defects with corresponding fusion functors [48]. This then

\footnotetext{
${ }^{7}$ Of course, tensoring a defect matrix factorisation $D$ always defines a functor in the category of matrix factorisations. The functors we are considering, however, act on the category of ring modules, and their action on a matrix factorisation $Q$ is simply given by applying $D$ on $Q$ seen as a ring module homomorphism.
} 
opens the possibility to study the fusion semi-ring of these defects. The fusion of rational defects is given by the rational fusion rules, and with the functorial description one can then identify the rational semi-ring structure also in the Landau-Ginzburg description. We will report on this in an upcoming publication [48].

After having the $\mathrm{SU}(3) / \mathrm{U}(2)$ model under control, one may ask whether a similar strategy also works for the higher rank models. Also in this case there exists a variable transformation interface to a product of minimal models [14], and the natural ansatz would be to study the effect of fusing it to known factorisations in the minimal models, maybe to the permutation factorisations of [11]. Although it is far from obvious, one might be lucky and generate in this way factorisations for rational boundary conditions or defects.

\section{Acknowledgments}

We thank Nils Carqueville, Dan Murfet and Ingo Runkel for helpful discussions. We would also like to thank the anonymous referee for the careful reading of the manuscript and for valuable comments and suggestions.

\section{A Similarity transformations}

For convenience we summarise here our conventions for the basic row and column transformations. For a matrix factorisation $Q$ of matrix size $2 d$ of the form

$$
Q=\left(\begin{array}{cc}
0 & Q^{(1)} \\
Q^{(0)} & 0
\end{array}\right)
$$

with the two $d \times d$ blocks $Q^{(0)}$ and $Q^{(1)}$, similarity transformations are given by invertible $2 d \times 2 d$ matrices $\mathcal{U}$ of the form

$$
\mathcal{U}=\left(\begin{array}{cc}
\mathcal{U}^{(0)} & 0 \\
0 & \mathcal{U}^{(1)}
\end{array}\right)
$$

They act on $Q$ as

$$
Q \mapsto \mathcal{U} \cdot Q \cdot \mathcal{U}^{-1}=\left(\begin{array}{cc}
0 & \mathcal{U}^{(0)} \cdot Q^{(1)} \cdot\left(\mathcal{U}^{(1)}\right)^{-1} \\
\mathcal{U}^{(1)} \cdot Q^{(0)} \cdot\left(\mathcal{U}^{(0)}\right)^{-1} & 0
\end{array}\right)
$$

The group of similarity transformations can be generated by elementary row and column transformations on $Q^{(1)}$ (which induce corresponding elementary column and row transformations on $\left.Q^{(0)}\right)$. For the basic operations we take

$$
\begin{aligned}
\mathcal{U}_{\text {row }}(r, s ; p)_{i j}:= & \delta_{i, j}+p \delta_{i, s} \delta_{j, r} \quad(p \text { any polynomial }) \\
& \text { adds row } r \text { multiplied by } p \text { to row } s \text { in } Q^{(1)} \\
& \text { adds column } s \text { multiplied by }-p \text { to column } r \text { in } Q^{(0)}
\end{aligned}
$$




$$
\begin{aligned}
\mathcal{U}_{\text {row } \times}(r ; \alpha)_{i j}:= & \delta_{i j}\left(1+\delta_{i, r}(\alpha-1)\right) \quad(\alpha \in \mathbb{C}) \\
& \text { multiplies row } r \text { with } \alpha \text { in } Q^{(1)} \\
& \text { multiplies column } r \text { with } 1 / \alpha \text { in } Q^{(0)} \\
\mathcal{U}_{\text {col }}(r, s ; p)_{i j}:= & \delta_{i, j}-p \delta_{i, r+d} \delta_{j, s+d} \quad(p \text { any polynomial }) \\
& \text { adds column } r \text { multiplied by } p \text { to column } s \text { in } Q^{(1)} \\
& \text { adds row } s \text { multiplied by }-p \text { to row } r \text { in } Q^{(0)} \\
\mathcal{U}_{\text {col× }}(r ; \alpha)_{i j}:= & \left.\delta_{i j}\left(1+\delta_{i, d+r}\left(\frac{1}{\alpha}-1\right)\right)\right) \quad(\alpha \in \mathbb{C}) \\
& \text { multiplies column } r \text { with } \alpha \text { in } Q^{(1)} \\
& \text { multiplies row } r \text { with } 1 / \alpha \text { in } Q^{(0)} .
\end{aligned}
$$

\section{B Multiple defect action on polynomial factorisations}

In this appendix we want to prove that the factorisations $Q_{n(m)}$ with upper right block

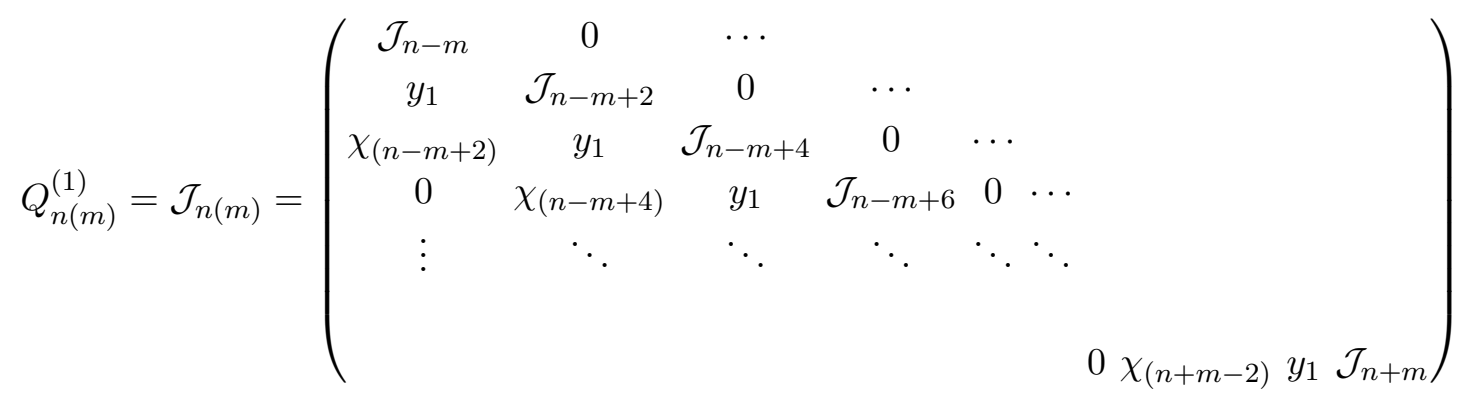

have the following behaviour when we apply the fusion functor $D_{(1)}$ :

$$
D_{(1)} Q_{n(m)} \cong Q_{n(m-1)} \oplus Q_{n(m+1)}
$$

where it is understood that $Q_{n(-1)}$ is omitted for $m=0$. We have proven this relation for $m=0$ and $m=1$ already in the main text. From the form (B.1) we see that the factorisations $Q_{n(m)}$ contain the factorisations $Q_{n-m}, \ldots, Q_{n+m}$ as building blocks. For $Q_{n}$ we have shown (see (4.42)) that

$$
D_{(1)}\left(Q_{n(0)}\right)=\mathcal{U}_{n}^{-1} \cdot Q_{n(1)} \cdot \mathcal{U}_{n}
$$


When we apply $D_{(1)}$ on $Q_{n(m)}$, we will apply the similarity transformations $\mathcal{U}_{j}$ on each block $D_{(1)}\left(Q_{j}\right)$ that appears. We find

$$
\begin{aligned}
& \mathcal{U}_{n(m)}^{a(0)} \cdot D_{(1)}\left(\mathcal{J}_{n(m)}\right) \cdot\left(\mathcal{U}_{n(m)}^{a(1)}\right)^{-1} \\
& =\left(\begin{array}{cccccc}
\mathcal{J}_{n-m(1)} & 0 & \ldots & & & \\
\Phi_{n-m+1}^{A} & \mathcal{J}_{n-m+2(1)} & 0 & \ldots & & \\
\Phi_{n-m+2}^{B} & \Phi_{n-m+3}^{A} & \mathcal{J}_{n-m+4(1)} & 0 & \ldots & \\
0 & \Phi_{n-m+4}^{B} & \Phi_{n-m+5}^{A} & \mathcal{J}_{n-m+6(1)} & 0 & \ldots \\
\vdots & \ddots & \ddots & \ddots & \ddots & \ddots \\
& & & & &
\end{array}\right.
\end{aligned}
$$

with the similarity transformation $\mathcal{U}_{n(m)}^{a}$ given by

$$
\mathcal{U}_{n(m)}^{a}=\prod_{j=0}^{m}{ }^{1+2 j} \mathcal{U}_{n-m+2 j}
$$

Here the left upper index on the $\mathcal{U}_{n}$ indicates on which row or column the transformation acts (see (4.43)). The blocks $\Phi_{j}^{A / B}$ are given by

$$
\Phi_{j}^{A}=\mathcal{U}_{j+1}^{(0)} \cdot D_{(1)}\left(y_{1}\right) \cdot\left(\mathcal{U}_{j-1}^{(1)}\right)^{-1}=\left(\begin{array}{cc}
y_{1} \frac{\pi_{2 j+3}}{\pi_{2 j+5}} & \mathcal{J}_{j} \frac{\mu_{2} \mu_{4}}{\pi_{2 j+5} \pi_{-2 j+3}} \\
\frac{1}{2 \pi_{2}} & y_{1} \frac{\pi_{-2 j+1}}{\pi_{-2 j+3}}
\end{array}\right)
$$

and

$$
\Phi_{j}^{B}=\mathcal{U}_{j+2}^{(0)} \cdot D_{(1)}\left(\chi_{(j)}\right) \cdot\left(\mathcal{U}_{j-2}^{(1)}\right)^{-1}=\left(\begin{array}{cc}
\frac{\pi_{2 j+5} \pi_{-2 j+3}}{4 \pi_{2}^{2} \pi_{-2 j+1} \pi_{2 j+7}} & -y_{1} \frac{\mu_{4} \mu_{-4} \pi_{2 j+5} \pi_{-2 j+3}}{4 \pi_{2}^{2} \pi_{2 j+7} \pi_{-2 j+5} \pi_{2 j-1} \pi_{-2 j-3}} \\
0 & \frac{\pi_{2 j+5} \pi_{-2 j+3}}{4 \pi_{2}^{2} \pi_{2 j+3} \pi_{-2 j+5}}
\end{array}\right)
$$

We now reorganise the result (B.4) into the block form

$$
\begin{aligned}
& \mathcal{U}_{n(m)}^{a(0)} \cdot D_{(1)}\left(\mathcal{J}_{n(m)}\right) \cdot\left(\mathcal{U}_{n(m)}^{a(1)}\right)^{-1}
\end{aligned}
$$

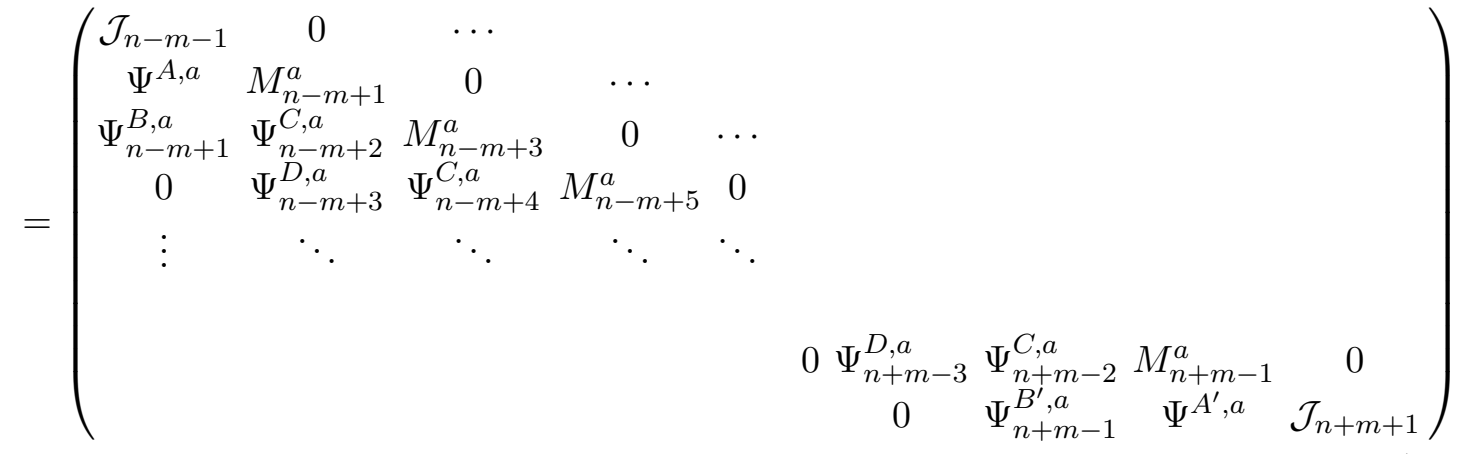


with

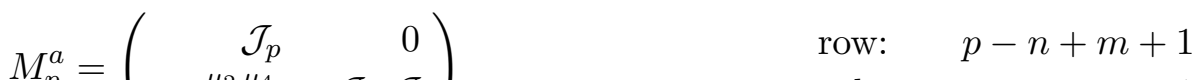

$$
\begin{aligned}
& \text { column: } p-n+m+1 \\
& (p=n-m-1+2 r, r=1, \ldots, m) \\
& \Psi^{A, a}=y_{1}\left(\begin{array}{c}
1 \\
\frac{\pi_{2 n-2 m+5}}{\pi_{2 n-2 m+7}}
\end{array}\right) \\
& \Psi^{A^{\prime}, a}=y_{1}\left(\frac{\pi_{2 n-2 m+5}}{\pi_{2 n-2 m+7}} 1\right) \\
& \Psi_{n-m+1}^{B, a}=\left(\begin{array}{c}
\frac{1}{2 \pi_{2}} \\
\frac{\pi_{2 n-2 m+9} \pi_{-2 n+2 m-1}}{4 \pi_{2}^{2} \pi_{-2 n+2 m-3} \pi_{2 n-2 m+11}}
\end{array}\right) \\
& \Psi_{n+m-1}^{B^{\prime}, a}=\left(\frac{\pi_{2 n+2 m+1} \pi_{-2 n-2 m+7}}{4 \pi_{2}^{2} \pi_{2 n+2 m-1} \pi_{-2 n-2 m+9}} \frac{1}{2 \pi_{2}}\right) \\
& \Psi_{p}^{C, a}=y_{1}\left(\begin{array}{cc}
\frac{\pi_{-2 p+3}}{\pi_{-2 p+5}} & 1 \\
-\frac{\mu_{4} \mu_{-4} \pi_{2 p+5} \pi_{-2 p+3}}{4 \pi_{2}^{2} \pi_{2 p+7} \pi_{-2 p+5} \pi_{2 p-1} \pi_{-2 p-3}} & \frac{\pi_{2 p+5}}{\pi_{2 p+7}}
\end{array}\right) \quad \begin{array}{l}
\text { row: } \\
\text { column: } p-n+m+2
\end{array} \\
& (p=n-m+2 r, r=1, \ldots, m-1) \\
& \Psi_{p}^{D, a}=\left(\begin{array}{cc}
\frac{\pi_{2 p+3} \pi_{-2 p+5}}{4 \pi_{2}^{2} \pi_{2 p+1} \pi_{-2 p+7}} & \frac{1}{2 \pi_{2}} \\
0 & \frac{\pi_{2 p+7} \pi_{-2 p+1}}{4 \pi_{2}^{2} \pi_{-2 p-1} \pi_{2 p+9}}
\end{array}\right) \quad \begin{array}{l}
\text { row: } p-n+m+3 \\
\end{array} \quad \begin{array}{l}
\text { column: } p-n+m-1 .
\end{array} \\
& (p=n-m+2 r+1, r=1, \ldots, m-2)
\end{aligned}
$$

Here we always stated the row and column number of the upper left entry of the given block.

Our strategy is now to eliminate all diagonal terms in the blocks $M, \Psi^{C}$ and $\Psi^{D}$ by similarity transformations (and the bottom/left entries in $\psi^{A}, \Psi^{B} / \Psi^{A^{\prime}}, \Psi^{B^{\prime}}$ ). If we can achieve this, the factorisation will split into a direct sum of two factorisations.

We start with the blocks $\Psi^{C}$. The similarity transformations

$$
\mathcal{U}_{n(m)}^{b}=\prod_{j=1}^{m}{ }^{2 j} \mathcal{U}_{n-m+2 j-1(1)}
$$

(see (4.50)) eliminate their diagonal entries,

$$
\begin{aligned}
\Psi_{p}^{C, b}= & { }^{p-n+m+2} \mathcal{U}_{p+1(1)}^{(0)} \cdot \Psi_{p}^{C, a} \cdot\left({ }^{p-n+m} \mathcal{U}_{p-1}^{(1)}\right)^{-1} \\
= & \mathcal{U}_{\mathrm{row}}^{(0)}\left(1,2 ;-\frac{\pi_{2 p+5}}{\pi_{2 p+7}}\right) \cdot y_{1}\left(\begin{array}{cc}
\frac{\pi_{-2 p+3}}{\pi_{-2 p+5}} & 1 \\
-\frac{\mu_{4} \mu_{-4} \pi_{2 p+5} \pi_{-2 p+3}}{4 \pi_{2}^{2} \pi_{2 p+7} \pi_{-2 p+5} \pi_{2 p-1} \pi_{-2 p-3}} & \frac{\pi_{2 p+5}}{\pi_{2 p+7}}
\end{array}\right) \\
& \cdot\left(\mathcal{U}_{\mathrm{col}}^{(1)}\left(2,1 ;-\frac{\pi_{-2 p+3}}{\pi_{-2 p+5}}\right)\right)^{-1} \\
= & y_{1}\left(\begin{array}{cc}
0 & 1 \\
\kappa_{p} & 0
\end{array}\right)
\end{aligned}
$$


with

$$
\begin{aligned}
\kappa_{p} & :=\frac{\delta}{4 \pi_{2}^{2} \chi_{(p-1)} \chi_{(p+1)}} \\
\delta & :=-\frac{1}{4 \pi_{2} \pi_{-2}}=-\frac{\eta^{2}}{\left(1+\eta^{2}\right)^{2}} .
\end{aligned}
$$

Also one of the entries in $\Psi^{A}$ and $\Psi^{A^{\prime}}$ is eliminated,

$$
\Psi^{A, b}=\left(\begin{array}{c}
y_{1} \\
0
\end{array}\right) \quad \Psi^{A^{\prime}, b}=\left(\begin{array}{ll}
0 & y_{1}
\end{array}\right)
$$

The effect on the other blocks is

$$
\begin{aligned}
M_{p}^{b} & =\left(\begin{array}{cc}
\mathcal{J}_{p} & 0 \\
-\frac{1}{2 \pi_{2} \chi_{(p)}} \mathcal{J}_{p} & \mathcal{J}_{p}
\end{array}\right) \\
\Psi_{n-m+1}^{B, b} & =\left(\begin{array}{cc}
\frac{1}{2 \pi_{2}} & \\
\kappa_{n-m+2} \chi_{(n-m+1)}
\end{array}\right) \quad \Psi_{n+m-1}^{B^{\prime}, b}=\left(\kappa_{n+m-2} \chi_{(n+m-1)} \frac{1}{2 \pi_{2}}\right) \\
\Psi_{p}^{D, b} & =\left(\begin{array}{cc}
\kappa_{p-1} \chi_{(p)} & \frac{1}{2 \pi_{2}} \\
0 & \kappa_{p+1} \chi_{(p)}
\end{array}\right) .
\end{aligned}
$$

We then turn to the blocks $M$ and apply the transformation

$$
\mathcal{U}_{n(m)}^{c}=\prod_{j=1}^{m}{ }^{2 j} \mathcal{U}_{n-m+2 j-1(1)}
$$

(see (4.51)), whose effect on the blocks $M_{p}$ is

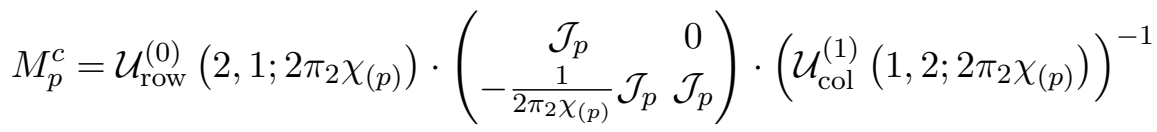

$$
\begin{aligned}
& =\left(\begin{array}{cc}
0 & 2 \pi_{2} \chi_{(p)} \mathcal{J}_{p} \\
-\frac{1}{2 \pi_{2} \chi_{(p)}} \mathcal{J}_{p} & 0
\end{array}\right) .
\end{aligned}
$$

We then rescale the entries by a further similarity transformation given by

$$
\begin{aligned}
\mathcal{U}_{n(m)}^{d}= & \prod_{j=1}^{m}\left\{\mathcal{U}_{\text {row } \times}\left(2 j ; \prod_{l=0}^{m-j} \frac{1}{2 \pi_{2} \chi_{(n+m-2 l-1)}}\right) \mathcal{U}_{\text {row } \times}\left(2 j-1 ; \prod_{l=0}^{m-j} \frac{1}{2 \pi_{2} \chi_{(n+m-2 l-1)}}\right)\right. \\
& \left.\cdot \mathcal{U}_{\text {col } \times}\left(2 j ; \prod_{l=0}^{m-j} 2 \pi_{2} \chi_{(n+m-2 l-1)}\right) \mathcal{U}_{\text {col } \times}\left(2 j-1 ; \prod_{l=0}^{m-j} 2 \pi_{2} \chi_{(n+m-2 l-1)}\right)\right\}, \quad(\mathrm{B} .
\end{aligned}
$$


and the blocks read after this transformation

$$
\begin{aligned}
& M_{p}^{d}=\left(\begin{array}{cc}
0 & \mathcal{J}_{p} \\
-\mathcal{J}_{p} & 0
\end{array}\right) \\
& \Psi^{A, d}=\left(\begin{array}{c}
y_{1} \\
0
\end{array}\right) \quad \Psi^{A^{\prime}, d}=\left(\begin{array}{ll}
0 & y_{1}
\end{array}\right) \\
& \Psi_{n-m+1}^{B, d}=\chi_{(n-m+1)}\left(\begin{array}{c}
1+\delta \\
\delta
\end{array}\right) \quad \Psi_{n+m-1}^{B^{\prime}, d}=\chi_{(n+m-1)}(\delta 1+\delta) \\
& \Psi_{p}^{C, d}=y_{1}\left(\begin{array}{cc}
\delta & 1+\delta \\
\delta & \delta
\end{array}\right) \\
& \Psi_{p}^{D, d}=\chi_{(p)}\left(\begin{array}{cc}
\delta & 1+2 \delta \\
0 & \delta
\end{array}\right) .
\end{aligned}
$$

For the next step we introduce another symbol, $\Delta_{p}$, that we define recursively by

$$
\Delta_{p+1}=1+\frac{\delta}{\Delta_{p}} \quad, \quad \Delta_{1}=1
$$

We now want to eliminate the lower right entries of $\Psi^{C}$ and $\Psi^{D}$, and we perform the similarity transformations

$$
\begin{aligned}
\mathcal{U}_{n(m)}^{e} & :=\prod_{r=1}^{m-1} \mathcal{U}_{r c}\left(2 r+2,2 r+3 ;-\frac{\delta}{\Delta_{r} \Delta_{r+1}}\right) \\
\mathcal{U}_{r c}(r, s ; p) & :=\mathcal{U}_{\text {col }}(r, s ; p) \cdot \mathcal{U}_{\text {row }}(r, s ; p)
\end{aligned}
$$

Because we do the same transformation on the rows and on the columns, the blocks $M_{p}^{d}$ will be left unchanged, $M_{p}^{e}=M_{p}^{d}$. The other blocks transform to

$$
\begin{aligned}
& \Psi^{A, e}=\left(\begin{array}{c}
y_{1} \\
0
\end{array}\right) \quad \Psi^{A^{\prime}, e}=\left(\begin{array}{ll}
0 & y_{1}
\end{array}\right) \\
& \Psi_{n-m+1}^{B, e}=\chi_{(n-m+1)}\left(\begin{array}{c}
1+\delta \\
0
\end{array}\right) \quad \Psi_{n+m-1}^{B^{\prime}, e}=\chi_{(n+m-1)}\left(\delta \Delta_{m}\right) \\
& \Psi_{n-m+2 r}^{C, e}=y_{1}\left(\begin{array}{cc}
\delta & \Delta_{r+1} \\
\frac{\delta}{\Delta_{r+1}} & 0
\end{array}\right) \\
& \Psi_{n-m+2 r+1}^{D, e}=\chi_{(n-m+2 r+1)}\left(\begin{array}{cc}
\delta & \Delta_{r+1} \Delta_{r+2} \\
-\frac{\delta^{2}}{\Delta_{r+1} \Delta_{r+2}} & 0
\end{array}\right) .
\end{aligned}
$$

To formulate our final similarity transformation we introduce the quantity $\gamma_{p, q}$ defined as

$$
\gamma_{p, q}:=\left(\prod_{i=1}^{q} \frac{\Delta_{p+i}}{\Delta_{i}}\right)
$$


It has the properties

$$
\begin{aligned}
\gamma_{p, q} & =\gamma_{q, p} \\
\gamma_{0, q} & =\gamma_{p, 0}=1 \\
\gamma_{1, q} & =\Delta_{q+1} \\
\gamma_{p, q-1} & =\frac{\Delta_{q}}{\Delta_{p}} \gamma_{p-1, q} \\
\gamma_{p, q} & =1+\frac{\delta}{\Delta_{p} \Delta_{q}} \quad(p, q \geq 1) .
\end{aligned}
$$

Whereas the first properties are obvious from the definition of $\gamma_{p, q}$, we present the proof of the last one:

We prove (B.28e) by induction. We first note that it is satisfied for $p=1$,

$$
\gamma_{1, q}=\Delta_{q+1}=1+\frac{\delta}{\Delta_{q}}=1+\frac{\delta}{\Delta_{1} \Delta_{q}}
$$

where we used the recursive definition of $\Delta_{q+1}$ (see (B.23)). Now assume that (B.28e) holds for some $p \geq 1$. Then

$$
\begin{aligned}
\gamma_{p+1, q} & =\frac{\Delta_{q+1}}{\Delta_{p+1}} \gamma_{p, q+1} \\
& =\frac{\Delta_{q+1}}{\Delta_{p+1}}\left(1+\frac{\delta}{\Delta_{p} \Delta_{q+1}}\right) \\
& =\frac{1}{\Delta_{p+1}}\left(\Delta_{q+1}+\frac{\delta}{\Delta_{p}}\right) \\
& =\frac{1}{\Delta_{p+1}}\left(\frac{\delta}{\Delta_{q}}+\Delta_{p+1}\right) \\
& =1+\frac{\delta}{\Delta_{p+1} \Delta_{q}} .
\end{aligned}
$$

We can finally formulate the transformation that will remove the remaining diagonal entry in $\Psi^{C}$ and $\Psi^{D}$, which is given by

$$
\mathcal{U}_{n(m)}^{f}:=\prod_{r=1}^{m-1} \mathcal{U}_{r c}\left(2 r+1,2 r ;-\frac{\delta}{\Delta_{r+1} \gamma_{r+1, m-r-1}}\right)
$$


We obtain

$$
\begin{array}{rlr}
M_{p}^{f} & =\left(\begin{array}{cc}
0 & \mathcal{J}_{p} \\
-\mathcal{J}_{p} & 0
\end{array}\right) & \\
\Psi^{A, f} & =\left(\begin{array}{c}
y_{1} \\
0
\end{array}\right) & \Psi^{A^{\prime}, f}=\left(\begin{array}{ll}
0 & y_{1}
\end{array}\right) \\
\Psi_{n-m+1}^{B, f} & =\chi_{(n-m+1)}\left(\begin{array}{c}
1+\delta \\
0
\end{array}\right) & \Psi_{n+m-1}^{B^{\prime}, f}=\chi_{(n+m-1)}\left(\begin{array}{ll}
0 & \Delta_{m}
\end{array}\right) \\
\Psi_{n-m+2 r}^{C, f} & =y_{1}\left(\begin{array}{cc}
0 & \Delta_{r+1} \\
\frac{\delta}{\Delta_{r+1}} & 0
\end{array}\right) \\
\Psi_{n-m+2 r+1}^{D, f} & =\chi_{(n-m+2 r+1)}\left(\begin{array}{cc}
0 & \Delta_{r+1} \Delta_{r+2} \\
-\frac{\delta^{2}}{\Delta_{r+1} \Delta_{r+2}} & 0
\end{array}\right) .
\end{array}
$$

We have thus achieved our goal to eliminate all diagonal terms in the blocks, and the factorisation can now be decomposed into two (see table 1 on page 41). Up to remaining multiplicative transformations of rows and columns, these two factorisations are precisely $Q_{n(m-1)}$ and $Q_{n(m+1)}$. This proves our claim.

\section{Deriving the alternative standard form}

In this section we want to sketch how we arrived at the alternative standard form $\tilde{Q}_{n(m)}$ given in (4.75). Recall that we want to successively apply $D_{(1)}$ on cones of polynomial factorisations with one elementary factor $\mathcal{J}_{p_{i}}$ and decompose the result using similarity transformations that leave the morphisms unchanged.

It turns out that to arrive at the alternative standard form, it is enough to look at cones of three polynomial factorisations,

$$
\mathcal{J}_{p, q, r}:=\left(\begin{array}{ccc}
\mathcal{J}_{p} & 0 & 0 \\
1 & \mathcal{J}_{q} & 0 \\
0 & 1 & \mathcal{J}_{r}
\end{array}\right) \quad(p, q, r \text { pairwise different })
$$

If we now apply $D_{(1)}$ successively, the morphism entries 1 will be mapped to identity matrices. We then perform similarity transformations and make sure that at the end the identity matrices are untouched.

In the first step we find

$$
D_{(1)}\left(\mathcal{J}_{p, q, r}\right)=\left(\begin{array}{ccc}
D_{(1)}\left(\mathcal{J}_{p}\right) & 0 & 0 \\
\mathbb{1} & D_{(1)}\left(\mathcal{J}_{q}\right) & 0 \\
0 & \mathbb{1} & D_{(1)}\left(\mathcal{J}_{r}\right)
\end{array}\right) .
$$

We then blockwise transform $D_{(1)}\left(\mathcal{J}_{n}\right)$ to $\tilde{\tilde{D}}_{0, m}\left(\mathcal{J}_{n}\right)$ as in $(4.70)$ to obtain

$$
D_{(1)}\left(\mathcal{J}_{p, q, r}\right) \cong\left(\begin{array}{ccc}
\tilde{\tilde{D}}_{0,1}\left(\mathcal{J}_{p}\right) & 0 & 0 \\
\mathbb{1} & \tilde{\tilde{D}}_{0,1}\left(\mathcal{J}_{q}\right) & 0 \\
0 & \mathbb{1} & \tilde{\tilde{D}}_{0,1}\left(\mathcal{J}_{r}\right)
\end{array}\right)
$$




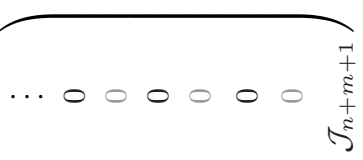

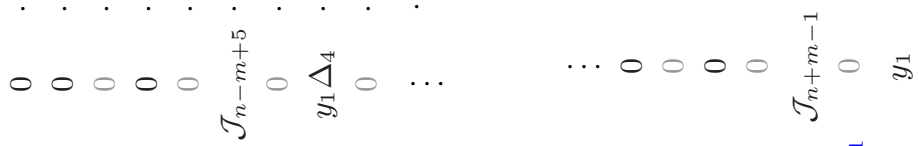

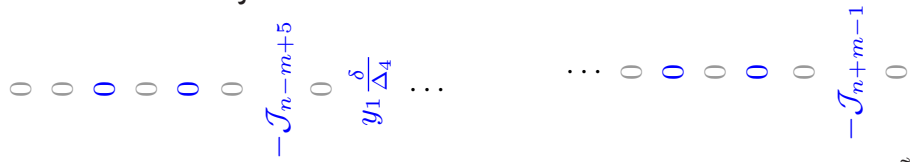

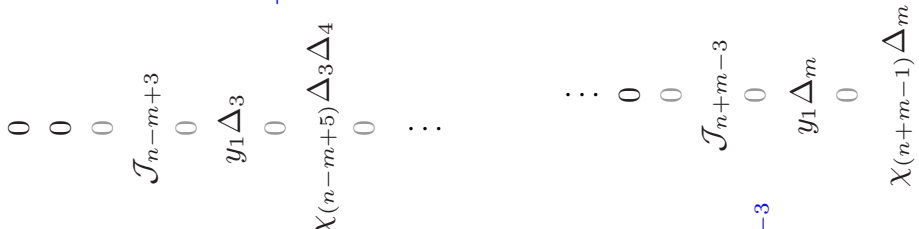

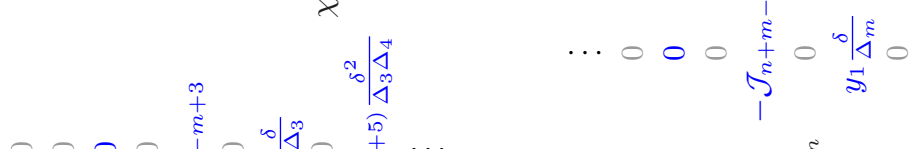

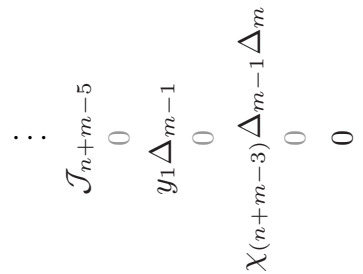

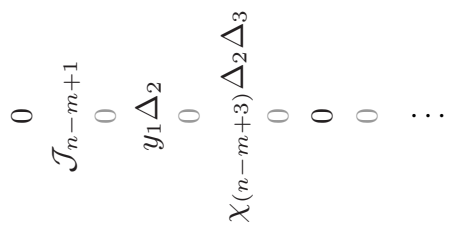

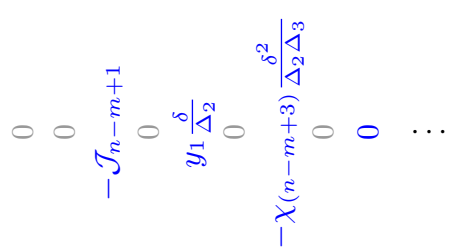

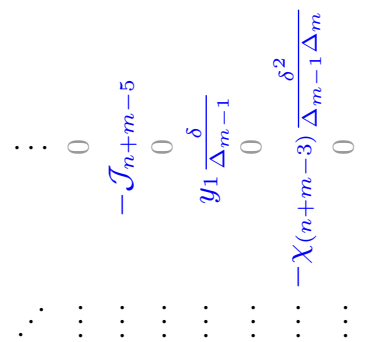

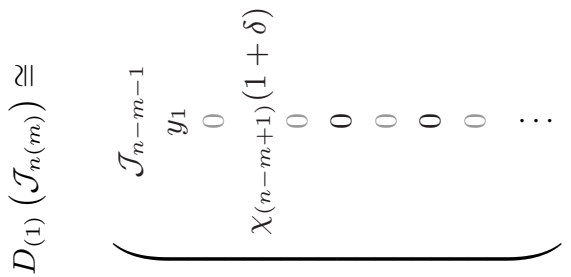

Table 1. The upper right block of the matrix factorisation $D_{(1)}\left(Q_{n(m)}\right)$ after the similarity transformations $\mathcal{U}_{n(m)}^{f} \cdots \mathcal{U}_{n(m)}^{a}$ - it can be decomposed into two parts: one (denoted in black) contains all entries in even lines (and in the first one) and in odd columns (and in the last one), and the other one (denoted in blue) consists of the complement. 
When we now apply $D_{(1)}$ again, we already should have an idea, which form we want to obtain for each block. As ingredients we take the blocks $\tilde{D}_{0, m}\left(\mathcal{J}_{n}\right)$ that have the nice property that their off-diagonal elements do not depend on $n$ (see (4.68)).

In the standard form $\mathcal{J}_{n(m)}$ introduced in section 4.5.1 we have on the diagonal the factors $\mathcal{J}_{n^{\prime}}$ with $n^{\prime}$ going monotonically from $n-m$ to $n+m$. We now have to reorder these entries, such that we can rewrite the expression in terms of the blocks $\tilde{D}_{0, m^{\prime}}\left(\mathcal{J}_{n}\right)$, which can be written as a cone of $\mathcal{J}_{n+m^{\prime}}$ and $\mathcal{J}_{n-m^{\prime}}$ in either direction,

$$
\begin{aligned}
\tilde{D}_{0, m}\left(\mathcal{J}_{n}\right) & =\mathcal{U}_{m ; n}^{(0)^{-1}} \cdot\left(\begin{array}{cc}
\mathcal{J}_{n-m} & 0 \\
y_{1} & \mathcal{J}_{n+m}
\end{array}\right) \cdot \mathcal{U}_{m ; n}^{(1)} \\
& =\mathcal{U}_{m ; n}^{(0)^{\dagger-1}} \cdot\left(\begin{array}{cc}
\mathcal{J}_{n+m} & 0 \\
y_{1} & \mathcal{J}_{n-m}
\end{array}\right) \cdot \mathcal{U}_{m ; n}^{(1)^{\dagger}}
\end{aligned}
$$

where

$$
\begin{aligned}
\mathcal{U}_{m ; n}:= & \mathcal{U}_{\times}\left(2,2 ; \frac{1}{2 \mu_{2 m} \pi_{2 m}}, 2 \mu_{2 m} \pi_{2 m}\right) \cdot \mathcal{U}_{\times}\left(1,2 ; \frac{\pi_{2 n-2 m+1}}{\pi_{2 n+2 m+1}}, \frac{\pi_{-2 m-2 n-1}}{\pi_{2 m-2 n-1}}\right) \\
& \cdot \mathcal{U}_{\mathrm{col}}\left(1,2 ;-\frac{y_{1} \mu_{2 n+2 m+1}}{\pi_{2 n+2 m+1}}\right) \cdot \mathcal{U}_{\text {row }}\left(2,1 ; \frac{y_{1} \mu_{2 n-2 m+1}}{\pi_{2 n-2 m+1}}\right) \\
\mathcal{U}_{m ; n}^{\dagger}:= & \mathcal{U}_{\times}\left(2,2 ; \frac{1}{2 \mu_{2 m} \pi_{2 m}}, 2 \mu_{2 m} \pi_{2 m}\right) \cdot \mathcal{U}_{\times}\left(1,2 ; \frac{\pi_{-2 m-2 n-1}}{\pi_{2 m-2 n-1}}, \frac{\pi_{2 n-2 m+1}}{\pi_{2 n+2 m+1}}\right) . \\
& \cdot \mathcal{U}_{\mathrm{col}}\left(1,2 ; \frac{y_{1} \mu_{2 n-2 m+1}}{\pi_{2 n-2 m+1}}\right) \cdot \mathcal{U}_{\text {row }}\left(2,1 ;-\frac{y_{1} \mu_{2 n+2 m+1}}{\pi_{2 n+2 m+1}}\right) \\
\mathcal{U}_{\times}(r, c ; \alpha, \beta):= & \mathcal{U}_{\mathrm{col} \times}(c ; \beta) \cdot \mathcal{U}_{\text {row } \times}(r ; \alpha) .
\end{aligned}
$$

In particular, these transformations allow us to "swap" the positions of any adjacent polynomial factors in our general formula for $\mathcal{J}_{n(m)}$. Focusing only on the diagonal and first lower sub-diagonal entries of $\mathcal{J}_{n(m)}$ for a moment, we can thus generate from $\mathcal{J}_{n(m)}$ factorisations with the (sub-)diagonal entry structure (the other lower diagonals have nontrivial entries)

$$
\mathcal{J}_{n(m)}=\left(\begin{array}{ccccc}
\mathcal{J}_{n-m} & & & & \\
y_{1} & \mathcal{J}_{n-m+2} & & & \\
& y_{1} & \mathcal{J}_{n-m+4} & & \\
& & \ddots & & \\
& & & \mathcal{J}_{n+m-2} & \\
& & & y_{1} & \mathcal{J}_{n+m}
\end{array}\right)
$$

an alternative form, in which the (sub-)diagonal entries read (again the other lower diago- 
nals have non-trivial entries)

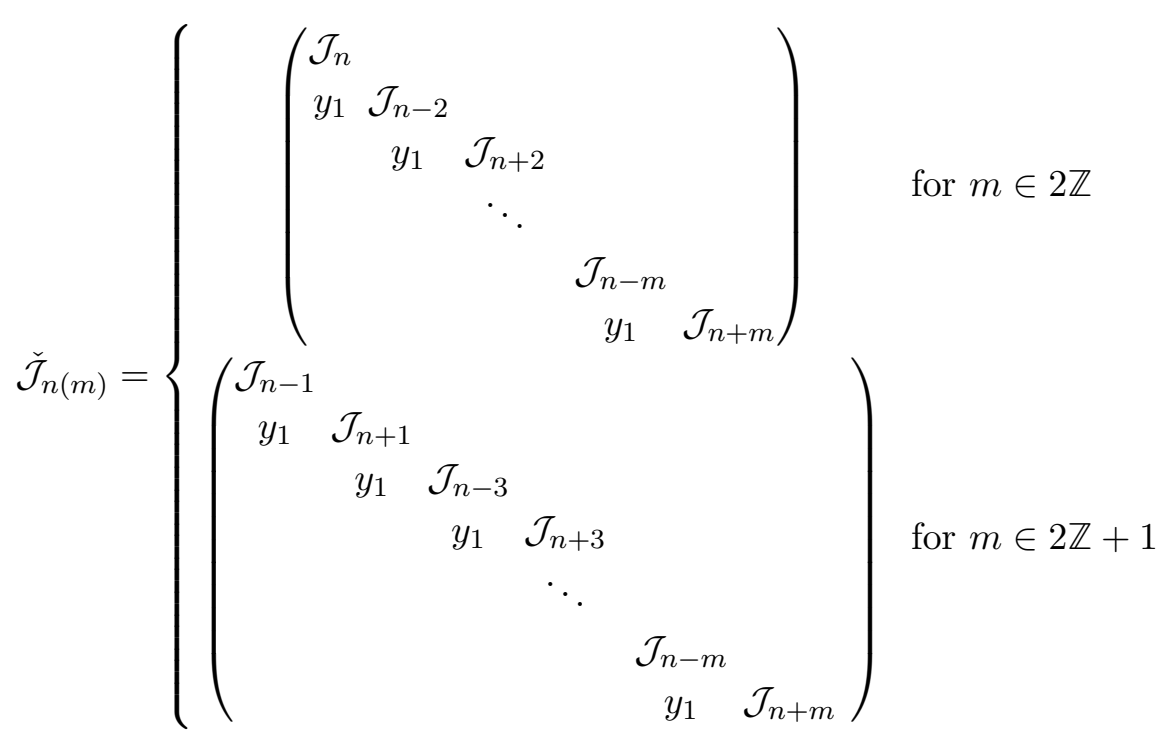

and which may be obtained via suitable combinations of the aforementioned similarity transformations. Unfortunately, in each intermediate step, after an application of two similarity transformations of the types listed in (C.5), we need to apply additional multiplicative transformations in order to ensure that the entries on the sub-diagonal all read $y_{1}$ (i.e. with constant prefactor 1). Postponing the resolution of this computational problem for the moment, we observe that once we have transformed $Q_{n(m)}$ into the form $\check{Q}_{n(m)}$, we can formulate yet another set of transformations (namely suitable inverse transformations of type (C.5)) to express all diagonal blocks in the form $\tilde{D}_{0, p}\left(\mathcal{J}_{n}\right)$ to obtain (note that again the lower non-diagonal blocks are non-trivial)

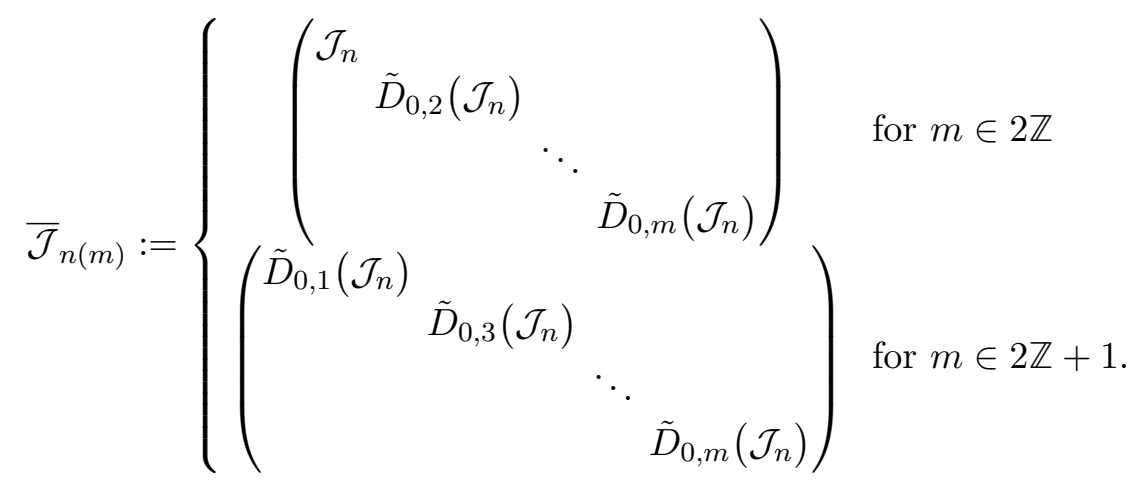

Having described the general strategy, we can now go into the concrete computations. The first step consists of computing $D_{(1)}\left(D_{(1)}\left(\mathcal{J}_{p, q, r}\right)\right)$ explicitly, that is via applying $D_{(1)}$ to $D_{(1)}\left(\mathcal{J}_{p, q, r}\right)$ in the form (C.3). Omitting the details of the rather tedious computation (the computation can be done in the framework of concatenations of fusion functors and will be presented in a more general setting in [48]), we obtain the decomposition

$$
D_{(1)}\left(D_{(1)}\left(Q_{p, q, r}\right)\right) \cong Q_{p, q, r} \oplus Q_{p, q, r(2)},
$$


where the summand $Q_{p, q, r(2)}$ is given by

$$
\mathcal{J}_{p, q, r(2)}=\left(\begin{array}{cccccc}
\eta^{4} \mathcal{J}_{p} & 0 & 0 & 0 & 0 & 0 \\
\Psi_{0,2} & \tilde{\tilde{D}}_{0,2}\left(\mathcal{J}_{p}\right) & 0 & 0 & 0 & 0 \\
1 & 0 & \eta^{4} \mathcal{J}_{q} & 0 & 0 & 0 \\
0 & \mathbb{1}_{2 \times 2} & \Psi_{0,2} & \tilde{\tilde{D}}_{0,2}\left(\mathcal{J}_{q}\right) & 0 & 0 \\
0 & 0 & 1 & 0 & \eta^{4} \mathcal{J}_{r} & 0 \\
0 & 0 & 0 & \mathbb{1}_{2 \times 2} & \Psi_{0,2} & \tilde{\tilde{D}}_{0,2}\left(\mathcal{J}_{r}\right)
\end{array}\right), \quad \Psi_{0,2}=\left(\begin{array}{c}
y_{1} \\
\frac{1}{4 \pi_{2}^{2} \pi_{4}}
\end{array}\right)
$$

We have thus achieved our goal of finding a new standard form $\tilde{\mathcal{J}}_{n(2)}$ for the three diagonal blocks,

$$
\tilde{\mathcal{J}}_{n(2)}=\left(\begin{array}{cc}
\eta^{4} \mathcal{J}_{n} & 0 \\
\Psi_{0,2} & \tilde{\tilde{D}}_{0,2}\left(\mathcal{J}_{n}\right)
\end{array}\right) .
$$

In a similar fashion, using the similarity transformations of type (C.5), we can determine the explicit formulae for $\mathcal{J}_{p, q, r(3)}$ and $\mathcal{J}_{p, q, r(4)}$ by means of a long computation, ${ }^{8}$ with the result that we find new standard forms $\tilde{\mathcal{J}}_{n(m)}$ for the diagonal subblocks such that the morphisms between these subblocks are simply unit matrices of size $(m+1) \times(m+1)$. The results we find for $m=3$ and $m=4$ fit into the following inductive structure:

$$
\begin{aligned}
& m=1: \quad \tilde{\mathcal{J}}_{n(1)}=\tilde{\tilde{D}}_{0,1}\left(\mathcal{J}_{n}\right) \\
& m=2: \quad \tilde{\mathcal{J}}_{n(2)}=\left(\begin{array}{cc}
\eta^{4} \mathcal{J}_{n} & 0 \\
\Psi_{0,2} & \tilde{\tilde{D}}_{0,2}\left(\mathcal{J}_{n}\right)
\end{array}\right), \quad \Psi_{0,2}=\left(\begin{array}{c}
y_{1} \\
\frac{1}{4 \pi_{2}^{2} \pi_{4}}
\end{array}\right) \\
& m=3: \quad \tilde{\mathcal{J}}_{n(3)}=\left(\begin{array}{cc}
\eta^{4} \tilde{\mathcal{J}}_{n(1)} & 0 \\
\Psi_{1,3} & \tilde{\tilde{D}}_{0,3}\left(\mathcal{J}_{n}\right)
\end{array}\right), \quad \Psi_{1,3}=\left(\begin{array}{cc}
\frac{1}{4 \pi_{4} \pi_{-2} \pi_{2}} & y_{1} \\
0 & \frac{1}{4 \pi_{4} \pi_{2} \pi_{6}}
\end{array}\right) \\
& m=4: \quad \tilde{\mathcal{J}}_{n(4)}=\left(\begin{array}{cc}
\eta^{4} \tilde{\mathcal{J}}_{n(2)} & 0 \\
0_{2 \times 1} \Psi_{2,4} & \tilde{\tilde{D}}_{0,4}\left(\mathcal{J}_{n}\right)
\end{array}\right), \quad \Psi_{2,4}=\left(\begin{array}{cc}
\frac{1}{4 \pi_{6} \pi_{-2} \pi_{4}} & y_{1} \\
0 & \frac{1}{4 \pi_{6} \pi_{2} \pi_{8}}
\end{array}\right) \\
& m=p: \quad \tilde{\mathcal{J}}_{n(p)}=\left(\begin{array}{cc}
\eta^{4} \tilde{\mathcal{J}}_{n(p-2)} & 0 \\
0_{2 \times(p-3)} & \Psi_{p-2, p} \\
\tilde{D}_{0, p}\left(\mathcal{J}_{n}\right)
\end{array}\right), \\
& \Psi_{p-2, p}=\left(\begin{array}{cc}
\frac{1}{4 \pi_{2 p-2} \pi_{-2} \pi_{2 p-4}} & y_{1} \\
0 & \frac{1}{4 \pi_{2 p-2} \pi_{2} \pi_{2 p}}
\end{array}\right),
\end{aligned}
$$

where for $m=4$ and in the last expression for $\tilde{\mathcal{J}}_{n(p)}$ we spelled out the size of the zero-block in the lower left for clarity.

We conjecture that this structure holds for all $m$ up to a possible truncation due to the finiteness of the level $k$. As we will discuss in the following appendix D we expect the formula to be valid for $m+|n| \leq k / 2$ if $k$ is even, and for $m \leq k+2$ if $k$ is odd.

\footnotetext{
${ }^{8}$ The main complication which makes these computations difficult in practice is not so much the part of the transformations necessary to transform each diagonal subblock of type $\mathcal{J}_{n(m)}$, but rather to find those transformation necessary in addition to bring the relative morphisms into the simple form of $(m+1) \times$ $(m+1)$ unit matrices. In particular, one encounters the proliferation of rather complicated combinations of elementary constants in the relative morphism entries.
} 


\section{Constraints at finite level}

We have to pay attention that all similarity transformations that we perform are welldefined and that we do not accidentally divide by zero. The coefficients we use, $\pi_{p}, \mu_{p}$, $\chi_{(p)}, \Delta_{p}$ and $\gamma_{p, q}$ are generically neither zero or infinite, but there might be special values where they lead to divergent expressions in the similarity transformations.

The coefficients $\pi_{p}$ and $\mu_{p}$ (defined in (4.35)) are always finite, but they can be zero:

$$
\pi_{(2 z+1)(k+3)}=0 \quad(z \in \mathbb{Z}), \quad \mu_{2 z(k+3)}=0 \quad(z \in \mathbb{Z}) .
$$

The coefficients $\chi_{(p)}$ defined in (4.52) can vanish,

$$
\chi_{(p)}=0 \text { for } p=\left(\frac{k+2}{2} \pm 2\right)+z(k+3) \quad(z \in \mathbb{Z}),
$$

and they can also diverge,

$$
\chi_{(p)}=\infty \text { for } p=\left(\frac{k+2}{2} \pm 1\right)+z(k+3) \quad(z \in \mathbb{Z}) .
$$

Note that $\chi_{(p)}$ is always regular and non-zero for odd level $k$.

The coefficients $\Delta_{p}$ defined in (B.23) can also vanish,

$$
\Delta_{p}=0 \text { for } p=k+2+z(k+3) \quad(z \in \mathbb{Z}),
$$

or diverge,

$$
\Delta_{p}=\infty \text { for } p=z(k+3) \quad(z \in \mathbb{Z}) .
$$

The analysis for $\gamma_{p, q}$ is a bit more complicated, but one can show that it is regular and non-vanishing as long as

$$
p, q \geq 0 \quad \text { and } \quad p+q \leq k+1 .
$$

We are now in the position to analyse when the similarity transformations $\mathcal{U}^{a}, \ldots, \mathcal{U}^{f}$ used in appendix B to decompose

$$
D_{(1)}\left(Q_{n(m)}\right) \cong Q_{n(m-1)} \oplus Q_{n(m+1)}
$$

are well-defined.

The transformations $\mathcal{U}^{e}$ and $\mathcal{U}^{f}$ are independent of the label $n$ (see (B.24) and (B.35)). They contain the inverse of $\Delta_{r}$ for $r=1, \ldots, m$ and also the inverse of $\gamma_{r+1, m-r-1}$ for $r=1, \ldots, m-1$. From the considerations above one finds that these quantities are well defined for $m \leq k+1$.

The transformations $\mathcal{U}^{a}, \ldots, \mathcal{U}^{d}$ contain inverses of $\pi_{2 p+1}$ and of $\chi_{(p)}$. One can observe immediately that they can never be singular for odd level $k$. For even $k$, however, we have to analyse the situation more carefully. As an example look at the transformation $\mathcal{U}^{d}$ (defined in (B.21)). It contains inverses of $\chi_{(p)}$ for $p=n+m-2 l-1$ where $l=0, \ldots, m-1$. For $m \geq 0$ the label $p$ satisfies $|p| \leq m+|n|-1$. We have seen before that $\chi_{(p)}$ is regular and finite for $|p| \leq \frac{k}{2}-2$, therefore all similarity transformations are certainly regular for 
$m+|n|+1 \leq \frac{k}{2}$. One can show that this condition suffices to guarantee that also the other transformations $\mathcal{U}^{a}, \mathcal{U}^{b}$ and $\mathcal{U}^{c}$ are regular.

We conclude that the formula for $Q_{n(m+1)}$ that we obtained from the decomposition is valid if

$$
\begin{aligned}
m+1+|n| & \leq \frac{k}{2} & & \text { for } k \text { even } \\
m+1 & \leq k+2 & & \text { for } k \text { odd } .
\end{aligned}
$$

Similarly we can ask what the restrictions are on the alternative standard form $\tilde{\mathcal{J}}_{n(m)}$ that is used for the factorisation corresponding to a general boundary state $|L, \ell\rangle$. Because we use amongst others the transformation to the the first standard form $\mathcal{J}_{n(m)}$, we expect the constraint $m+|n| \leq k / 2$ for even $k$ and $m \leq k+2$ for odd $k$. One can check that also the additional transformations like the transformations (C.5) to swap the entries on the diagonal are well-defined if these conditions are satisfied. As the factorisation for $|L, \ell\rangle$ is built from cones of $\tilde{\mathcal{J}}_{0(\ell)}, \ldots, \tilde{\mathcal{J}}_{L(\ell)}$ we expect no constraints for $k$ odd (because $\ell \leq k+1$ for all boundary conditions), but for even $k$ we get the constraint $L+\ell \leq k / 2$. On the other hand we have the suspicion that one can also arrive at the alternative standard form $\tilde{\mathcal{J}}_{n(m)}$ by blockwise similarity transformations that do not depend on the label $n$. If this is true, then the constraint could also not depend on the label $n$, and for even $k$ we would simply obtain the constraint $m \leq k / 2$. In that case the formula for the factorisation for $|L, \ell\rangle$ would be correct for all $\ell \leq k / 2$.

\section{E A closed $2 \times 2$ form for $\mathcal{E}_{n(m)}$ and $\mathcal{J}_{n(m)}$}

In the main text we only considered the upper right block $Q^{(1)}$ (that we often denote by $\mathcal{J}$ ) of the matrix factorisations. The other block $Q^{(0)}$ (that we often denote by $\mathcal{E}$ ) can be reconstructed from $Q^{(1)}$ by

$$
Q^{(0)}=W_{2 ; k} \cdot\left(Q^{(1)}\right)^{-1}
$$

Since the matrix factorisations $Q_{n(m)}^{(1)}$ have a simple triangular structure, it is a straightforward recursive problem to determine the inverse that we describe in the following.

We start by writing explicitly the matrix elements of $\mathcal{J}_{n(m)}$,

$$
\mathbb{J}_{i}{ }^{j} \equiv\left(\mathcal{J}_{n(m)}\right)_{i}{ }^{j}=\delta_{i j} \mathcal{J}_{n-m+2(i-1)}+\delta_{i-1, j} y_{1}+\delta_{i-2, j} \chi_{(n-m+2 j)},
$$

where we introduced the shorthand notation $\mathbb{J}_{i}{ }^{j}$ for the components of $\mathcal{J}_{n(m)}$ for notational brevity in the ensuing computations. The indices $i, j$ run from 1 to $m+1$.

Introducing the additional shorthand notation

$$
\mathbb{E}_{i}^{j} \equiv\left(\mathcal{E}_{n(m)}\right)_{i}{ }^{j},
$$

we thus obtain an equation from which we can recursively determine the structure of $\mathcal{E}_{n(m)}$ (we write here and in the following $W \equiv W_{2 ; k}^{y}$ for brevity):

$$
\begin{aligned}
\mathcal{J}_{n(m)} \cdot \mathcal{E}_{n(m)} & =W \mathbb{1} \\
\Leftrightarrow \quad J_{i}{ }^{k} \mathbb{E}_{k}^{j} & =\left(\delta_{i k} \mathcal{J}_{n-m+2(k-1)}+\delta_{i-1, k} y_{1}+\delta_{i-2, k} \chi_{(n-m+2 k)}\right) \mathbb{E}_{k}^{j}=W \delta_{i j} .
\end{aligned}
$$


Obviously, $\mathcal{E}_{n(m)}$ is of lower triangular form,

$$
\mathbb{E}_{i}^{i+p}=0 \text { for } p>0 .
$$

The first non-trivial set of equations (for $i=j$ ) is

$$
\begin{aligned}
& \mathcal{J}_{n-m+2(i-1)} \mathbb{E}_{i}^{i}+\delta_{i-1, k} y_{1} \mathbb{E}_{k}^{i}+\delta_{i-2, k} \chi_{(n-m+2 k)} \mathbb{E}_{k}^{i}=W \\
\Leftrightarrow & \mathbb{E}_{i}^{i}=\frac{W}{\mathcal{J}_{n-m+2(i-1)}}
\end{aligned}
$$

where we used in the second line that $\delta_{i-1, k} \mathbb{E}_{k}^{i}=\delta_{i-2, k} \mathbb{E}_{k}^{i}=0$. The next special case is $i=j+1$, for which we obtain:

$$
\begin{aligned}
& \left(\delta_{j+1, k} \mathcal{J}_{n-m+2(k-1)}+\delta_{j, k} y_{1}+\delta_{j-1, k} \chi_{(n-m+2 k)}\right) \mathbb{E}_{k}{ }^{j}=0 \\
\Leftrightarrow & \mathbb{E}_{j+1}{ }^{j}=-\frac{y_{1} W}{\mathcal{J}_{n-m+2(j-1)} \mathcal{J}_{n-m+2 j}},
\end{aligned}
$$

where we made use of the result $\delta_{j-1, k} \mathbb{E}_{k}{ }^{j}=0$ yet again.

For $i=j+2+p\left(\right.$ with $p \in \mathbb{Z}_{\geq 0}$ ), we obtain a double recursion relation

$$
\begin{aligned}
& \left(\delta_{(j+2+p), k} \mathcal{J}_{n-m+2(j+p+1)}+\delta_{j+p+1, k} y_{1}+\delta_{j+p, k} \chi_{(n-m+2(j+p))}\right) \mathbb{E}_{k}{ }^{j}=0 \\
\Leftrightarrow & \mathbb{E}_{j+p+2}{ }^{j}=-\frac{1}{\mathcal{J}_{n-m+2(j+p+1)}}\left(y_{1} \mathbb{E}_{j+p+1}{ }^{j}+\chi_{(n-m+2(j+p))} \mathbb{E}_{j+p}{ }^{j}\right),
\end{aligned}
$$

which relates the entries in the $p+2^{\text {nd }}$ lower diagonal to the entries in the two diagonals above. Besides the dependence on the recursion parameter $p$, the factors in the recursion relation only depend on the combination $n-m+2 j$, so we introduce the notation $\Psi_{l(p)}$ for the entries $\mathbb{E}_{i}^{j}$ of $\mathcal{E}_{n(m)}$ defined by

$$
\Psi_{n-m+2 j+p-2(p+2)}:=\left(\mathcal{E}_{n(m)}\right)_{j+p+2}^{j} .
$$

The recursion relation then reads

$$
\Psi_{l(p)}=-\frac{1}{\mathcal{J}_{l+p}}\left(y_{1} \Psi_{l-1(p-1)}+\chi_{(l+p-2)} \Psi_{l-2(p-2)}\right)
$$

with

$$
\Psi_{l(1)}=-y_{1} \frac{W}{\mathcal{J}_{l-1} \mathcal{J}_{l+1}}, \quad \Psi_{l(0)}=\mathcal{E}_{l}:=\frac{W}{\mathcal{J}_{l}} .
$$


The first few solutions for $\mathcal{E}_{n(m)}$ are given by

$$
\begin{aligned}
& \mathcal{E}_{n(0)}=\left.\mathbb{E}_{1}{ }^{1}\right|_{m=0, n=n}=\mathcal{E}_{n}=\frac{W}{\mathcal{J}_{n}} \\
& \mathcal{E}_{n(1)}=\left(\begin{array}{ll}
\mathcal{E}_{n-1} & 0 \\
\Psi_{n(1)} & \mathcal{E}_{n+1}
\end{array}\right) \Rightarrow \Psi_{n(1)}=\left.\mathbb{E}_{2}{ }^{1}\right|_{m=1, n=n}=-\frac{y_{1} W}{\mathcal{J}_{n-1} \mathcal{J}_{n+1}} \\
& \mathcal{E}_{n(2)}=\left(\begin{array}{ccc}
\mathcal{E}_{n-2} & 0 & 0 \\
\Psi_{n-1(1)} & \mathcal{E}_{n} & 0 \\
\Psi_{n(2)} & \Psi_{n+1(1)} & \mathcal{E}_{n+2}
\end{array}\right) \\
& \Rightarrow \Psi_{n(2)}=\left.\mathbb{E}_{3}{ }^{1}\right|_{m=2, n=n}=-\left.\frac{1}{\mathcal{J}_{n+2}}\left(y_{1} \mathbb{E}_{2}{ }^{1}+\chi_{(n)} \mathbb{E}_{1}{ }^{1}\right)\right|_{m=2, n=n} \\
& =-\frac{1}{\mathcal{J}_{n+2}}\left(y_{1}\left(-\frac{y_{1} W}{\mathcal{J}_{n-2} \mathcal{J}_{n}}\right)+\chi_{(n)} \frac{W}{\mathcal{J}_{n-2}}\right) \\
& =-\frac{1}{\mathcal{J}_{n+2}}\left(y_{1} \Psi_{n-1(1)}+\chi_{(n)} \mathcal{E}_{n-2}\right) \\
& =\frac{W}{\mathcal{J}_{n-2} \mathcal{J}_{n} \mathcal{J}_{n+2}}\left(y_{1}^{2}-\chi_{(n)} \mathcal{J}_{n}\right) \\
& \mathcal{E}_{n(3)}=\left(\begin{array}{cccc}
\mathcal{E}_{n-3} & 0 & 0 & 0 \\
\Psi_{n-2(1)} & \mathcal{E}_{n-1} & 0 & 0 \\
\Psi_{n-1(2)} & \Psi_{n(1)} & \mathcal{E}_{n+1} & 0 \\
\Psi_{n(3)} & \Psi_{n+1(2)} & \Psi_{n+2(1)} & \mathcal{E}_{n+3}
\end{array}\right) \\
& \Rightarrow \Psi_{n(3)}=\left.\mathbb{E}_{4}{ }^{1}\right|_{m=3, n=n}=-\left.\frac{1}{\mathcal{J}_{n+3}}\left(y_{1} \mathbb{E}_{3}{ }^{1}+\chi_{(n+1)} \mathbb{E}_{2}{ }^{1}\right)\right|_{m=3, n=n} \\
& =-\frac{1}{\mathcal{J}_{n+3}}\left(y_{1} \Psi_{n-1(2)}+\chi_{(n+1)} \Psi_{n-2(1)}\right) \\
& =-\frac{y_{1} W}{\mathcal{J}_{n-3} \mathcal{J}_{n-1} \mathcal{J}_{n+1} \mathcal{J}_{n+3}}\left(y_{1}^{2}-\chi_{(n-1)} \mathcal{J}_{n-1}-\chi_{(n+1)} \mathcal{J}_{n+1}\right)
\end{aligned}
$$

We want to obtain the general solution to the recursion relation (E.10). We observe that in each recursion step for $\Psi_{l(p)}$ we either go one step down in $p$ and pick up a factor $-y_{1} / \mathcal{J}_{l+p}$ or we go two steps down in $p$ and pick up a factor

$$
-\frac{1}{\mathcal{J}_{l+p}} \chi_{(l+p-2)}=-\frac{1}{\mathcal{J}_{l+p} \mathcal{J}_{l+p-2}} \chi_{(l+p-2)} \mathcal{J}_{l+p-2} .
$$

The recursion ends when we reach $p=0$. Therefore we can have at most $\lfloor p / 2\rfloor$ factors of $\chi$ in $\Psi_{l(p)}$. We call $\Psi_{l(p)}^{(r)}$ the contribution to $\Psi_{l(p)}$ with $r$ factors of $\chi$, such that

$$
\Psi_{l(p)}=\sum_{r=0}^{\lfloor p / 2\rfloor} \Psi_{l(p)}^{(r)}
$$


When we go down always along the one-step recursion we have no factor of $\chi$ and we get the contribution

$$
\Psi_{l(p)}^{(0)}=\prod_{j=0}^{p-1}\left(-\frac{y_{1}}{\mathcal{J}_{l+p-2 j}}\right) \frac{W}{\mathcal{J}_{l-p}}=W \prod_{j=0}^{p} \frac{1}{\mathcal{J}_{l+p-2 j}}\left(-y_{1}\right)^{p} .
$$

When at one point we perform a two-step jump we get two factors of $y_{1}$ less and instead a factor of $-\chi_{\left(l-p+2 m_{1}\right)} \mathcal{J}_{l-p+2 m_{1}}$ with $m_{1}=1, \ldots, p-1$ depending on where we do the two-step jump, so the contribution $\Psi_{l(p)}^{(1)}$ is

$$
\Psi_{l(p)}^{(1)}=W \prod_{j=0}^{p} \frac{1}{\mathcal{J}_{l+p-2 j}}\left(-y_{1}\right)^{p-2} \sum_{m_{1}=1}^{p-1}\left(-\chi_{\left(l-p+2 m_{1}\right)} \mathcal{J}_{l-p+2 m_{1}}\right) .
$$

When we follow a two-step jump twice, we get again two factors of $y_{1}$ less, and instead a factor of $\chi_{\left(l-p+2 m_{2}\right)} \mathcal{J}_{l-p+2 m_{2}}$ more. Note, however that the difference of $m_{1}$ and $m_{2}$ has to be at least 2 because of course in a two-step jump we went two steps down. These arguments can easily be generalised to arbitrary numbers $r$ of factors of $\chi$ and we find in total the result

$$
\Psi_{l(p)}=W \prod_{j=0}^{p} \frac{1}{\mathcal{J}_{l+p-2 j}} \sum_{r=0}^{\lfloor p / 2\rfloor}\left(-y_{1}\right)^{p-2 r}(-1)^{r} \sum_{\substack{m_{1}, \ldots, m_{r} \\ m_{i}+1<m_{i+1} \\ 1 \leq m_{i} \leq p-1}} \prod_{i=1}^{r} \chi_{\left(l-p+2 m_{i}\right)} \mathcal{J}_{l-p+2 m_{i}} .
$$

As an aside we mention a graphical way of organising the different contributions. Introduce the analogue of a vacuum state for a spin chain, i.e. a state $|0\rangle_{h}$ with $h$ "holes", represented graphically as

$$
|0\rangle_{h} \widehat{=} \circ_{1} \circ_{2} \cdots \circ_{h-1} \circ_{h} \text {. }
$$

Then define the "creation" and "annihilation" operators $g_{i}^{+}$and $g_{i}^{-}$via

$$
\begin{aligned}
& g_{i}^{+} \cdots *_{i} \cdots:= \begin{cases}\cdots \bullet_{i} \cdots & \text { if } *_{i}=o_{i} \\
0 & \text { else }\end{cases}
\end{aligned}
$$

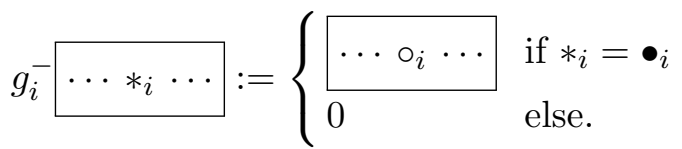

Also, we need to implement the rule that we may never have two neighbouring "excitations" $\bullet_{p} \bullet_{p+1}$. Together with the preceding definitions, we may compactly express these requirements as $(\forall i)$

$$
\begin{aligned}
g_{i}^{+} \circ g_{i}^{+} & =g_{i}^{-} \circ g_{i}^{-}=0 \\
g_{i}^{+} \circ g_{i}^{-} & =g_{i}^{-} \circ g_{i}^{+}=i d \\
g_{i}^{+} \circ g_{j}^{-} & =g_{j}^{-} \circ g_{i}^{+} \quad(i \neq j) \\
g_{i}^{+} \circ g_{i+1}^{+} & =g_{i+1}^{+} \circ g_{i}^{+}=0 \\
g_{i}^{+} \circ g_{i+2+p}^{+} & =g_{i+2+p}^{+} \circ g_{i}^{+} \quad(p \geq 0) .
\end{aligned}
$$


We may now define the operator $G$ as

$$
G=G_{1}+G_{2}, \quad G_{1}:=\sum_{i=1}^{\left\lfloor\frac{h+1}{2}\right\rfloor} g_{2 i-1}^{+}, \quad G_{2}:=\sum_{i=1}^{h-1} R_{i}
$$

where

$$
R_{i}:=g_{i}^{-} \circ g_{i+1}^{+}
$$

is the operator that moves an "excitation" $\bullet_{i}$ one position to the right (unless of course if we have another "excitation" sitting at position $i+2$, in which case $R_{i}$ annihilates the given state). This allows us finally to generate all possible states $|\Psi\rangle_{h}$ via repeated action of the operator $G$ on the "vacuum" state $|0\rangle_{h}$ - to this end, take the sum over arbitrary numbers of applications of $G$ (i.e. over $G^{n}$ ) applied to $|0\rangle_{h}$, and discard the multiplicities in this sum, ${ }^{9}$

$$
P_{h}:=\left.\left(\sum_{n=0}^{\infty} G^{n}|0\rangle_{h}\right)\right|_{\text {discard multiplicities }}=\sum_{m}\left|\Psi_{m}\right\rangle_{h},
$$

where $\left|\Psi_{m}\right\rangle_{h}$ denotes the inequivalent "excited" states. We will also need the operator $N$ which measures the number of "excitations" in a given state,

$$
N\left(\left|\Psi_{m}\right\rangle_{h}\right) \equiv N\left(g_{m_{1}}^{+} \circ g_{m_{2}}^{+} \circ \ldots \circ g_{m_{p}}^{+}|0\rangle_{h}\right):=\left|\left\{m_{1}, m_{2}, \ldots\right\}\right|=p
$$

with $|\ldots|$ denoting the cardinality of the set $\left\{m_{1}, m_{2}, \ldots\right\}$. Finally, we define the evaluation operator

$$
\begin{aligned}
e v_{n}\left(|0\rangle_{h}\right) & :=1 \\
e v_{n}\left(\left|\Psi_{m}\right\rangle_{h}\right) & \equiv e v_{n}\left(g_{m_{1}}^{+} \circ g_{m_{2}}^{+} \circ \ldots \circ g_{m_{p}}^{+}|0\rangle_{h}\right) \\
& :=\prod_{j=1}^{p} \chi_{\left(n-h-1+2 m_{j}\right)} \mathcal{J}_{n-h-1+2 m_{j}} \quad(p>0) .
\end{aligned}
$$

We can then rewrite the solution to $\Psi_{l(p)}$ as

$$
\Psi_{l(p)}=W \prod_{j=0}^{p} \frac{1}{\mathcal{J}_{l+p-2 j}} \sum_{r=0}^{\lfloor p / 2\rfloor}\left(-y_{1}\right)^{p-2 r}(-1)^{r}{ }_{l}^{r} M_{p-1}
$$

with

$$
{ }_{n}^{r} M_{h}=\sum_{N\left(\left|\Psi_{m}\right\rangle_{h}\right)=r} e v_{n}\left(\left|\Psi_{m}\right\rangle_{h}\right)
$$

For example, the graphical representation of the set of states with two "excitations" (i.e. $N=2$ ) at $h=6$ (together with the various possibilities to generate the set $\left\{\left|\Psi_{m}\right\rangle_{h} \mid\right.$ $\left.N\left(\left|\Psi_{m}\right\rangle_{h}\right)=2\right\}$ from one of its representatives is depicted in figure 2, while figure 3 represents the case $h=7$ and $N=3$.

\footnotetext{
${ }^{9}$ It is obvious that only finitely many non-zero states can arise in this sum, since we only have finitely many sites in a given state $|0\rangle_{h}$, and thus we obtain only finitely many possibilities to excite a given vacuum state.
} 
The results above allow us actually to derive a compact $2 \times 2$ realisation of $\mathcal{E}_{n(m)}$ and $\mathcal{J}_{n(m)}$. Consider the structure for $\mathcal{J}_{n(m)}$ as presented in (4.55). We may obviously choose to apply a number of row and column operations on $\mathcal{J}_{n(m)}$ in such a way that all rows and columns that intersect at a constant entry $\chi_{(p)}$ are "cleared out", to leave ultimately a form for $\mathcal{J}_{n(m)}$ of the form

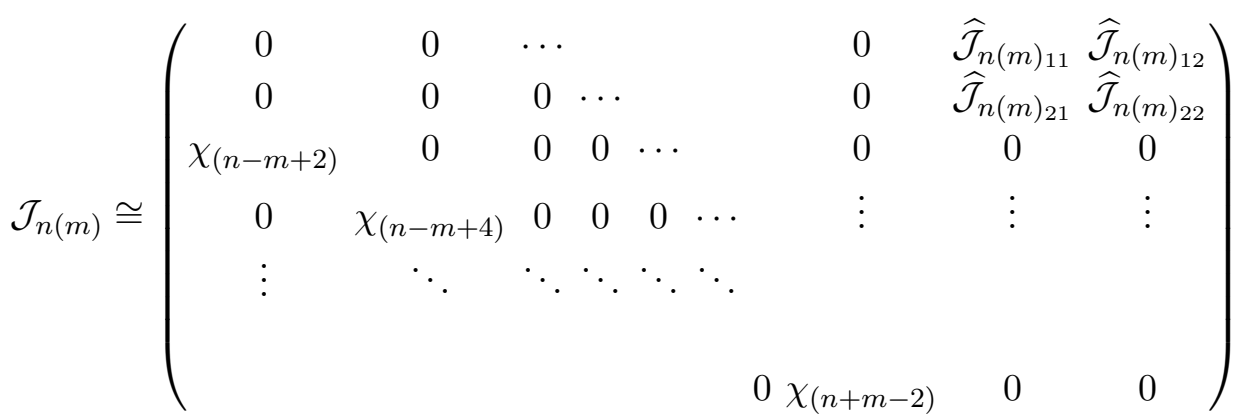

$$
\begin{aligned}
& \cong\left(\mathcal{J}_{\text {triv }}\right)^{\oplus(m-1)} \oplus \widehat{\mathcal{J}}_{n(m)} \text {. }
\end{aligned}
$$

Now, the structure of $\widehat{\mathcal{J}}_{n(m)}$ will generically become very complicated for large values of $m$, but we claim that

$$
\widehat{\mathcal{J}}_{n(m)} \widehat{\mathcal{E}}_{n(m)}=W \cdot \mathbf{1}_{2 \times 2}
$$

where $\widehat{\mathcal{E}}_{n(m)}$ is the lower left $2 \times 2$ subblock of $\mathcal{E}_{n(m)}$,

$$
\widehat{\mathcal{E}}_{n(m)}:=\left(\begin{array}{cc}
\Psi_{n-1(m-1)} & \Psi_{n(m-2)} \\
\Psi_{n(m)} & \Psi_{n+1(m-1)}
\end{array}\right) .
$$

We will prove this statement below. This result also allows us to give an explicit result for the $2 \times 2$ matrix $\widehat{\mathcal{J}}_{n(m)}$ as

$$
\widehat{\mathcal{J}}_{n(m)}=W\left(\widehat{\mathcal{E}}_{n(m)}\right)^{-1}=\frac{W}{\operatorname{det} \widehat{\mathcal{E}}_{n(m)}}\left(\begin{array}{cc}
\Psi_{n+1(m-1)} & -\Psi_{n(m-2)} \\
-\Psi_{n(m)} & \Psi_{n-1(m-1)}
\end{array}\right) .
$$

The determinant of $\widehat{\mathcal{E}}_{n(m)}$ can be obtained as follows. From the form of $\mathcal{J}_{n(m)}$ in (4.55) it is obvious that

$$
\operatorname{det} \mathcal{J}_{n(m)}=\prod_{j=0}^{m} \mathcal{J}_{n-m+2 j}
$$

When we perform the column and row manipulations to obtain the form (E.32), we do not change the determinant,${ }^{10}$ so from (E.32) we see that

$$
\operatorname{det} \mathcal{J}_{n(m)}=\operatorname{det} \widehat{\mathcal{J}}_{n(m)} \cdot \prod_{j=1}^{m-1} \chi_{n-m+2 j}
$$

\footnotetext{
${ }^{10}$ Note that to arrive at (E.32) we only performed transformations where we added multiples of rows (colums) to other rows (columns) and we did not rescale any row (column), so that the determinant remains unchanged.
} 


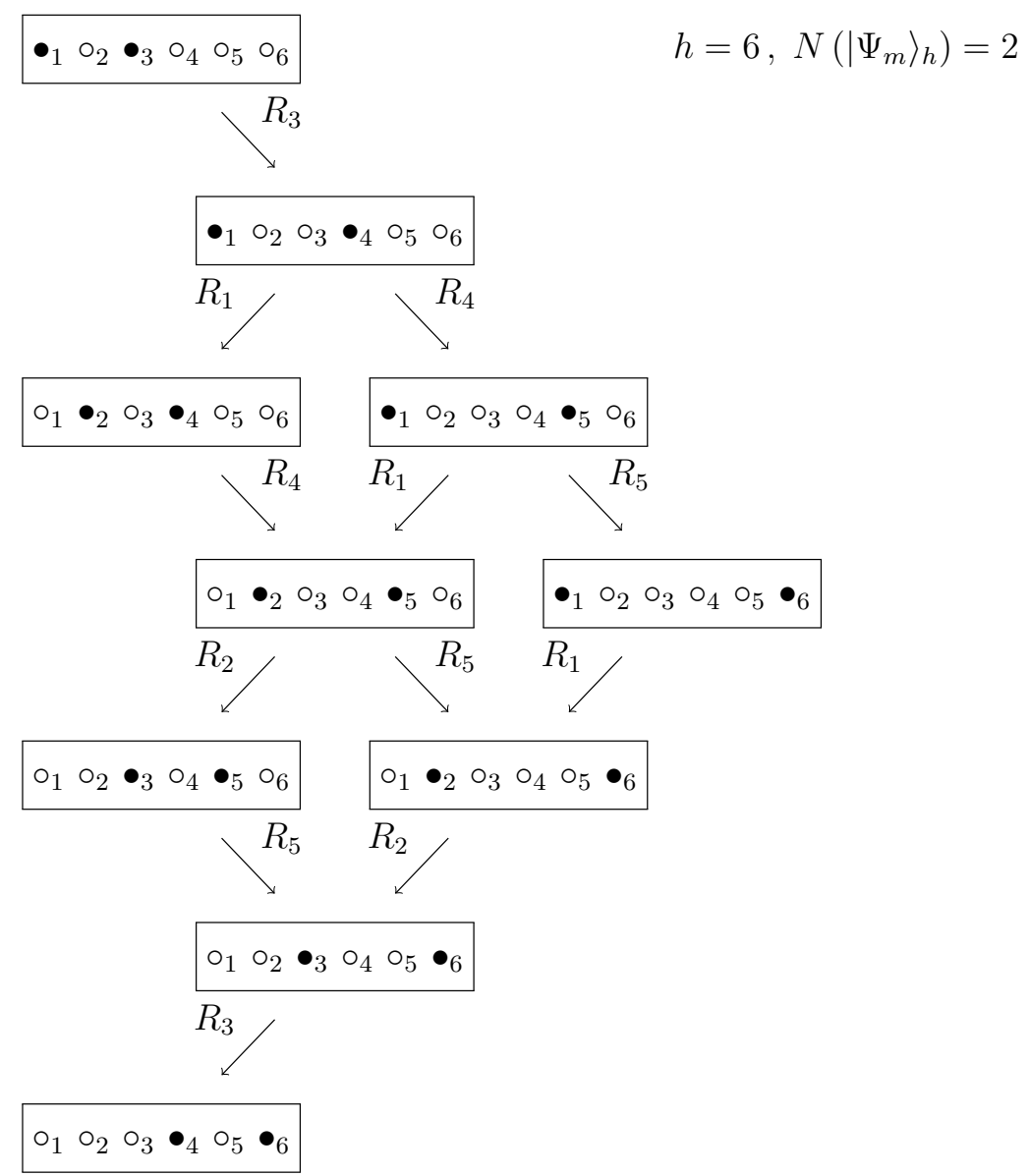

Figure 2. The case $h=6$ and $N=2$.

On the other hand, according to (E.34)

$$
\operatorname{det} \widehat{\mathcal{J}}_{n(m)} \cdot \operatorname{det} \widehat{\mathcal{E}}_{n(m)}=W^{2},
$$

which leads to

$$
\frac{W}{\operatorname{det} \widehat{\mathcal{E}}_{n(m)}}=\frac{1}{W} \frac{\prod_{j=0}^{m} \mathcal{J}_{n-m+2 j}}{\prod_{j=1}^{m-1} \chi_{(n-m+2 j)}} .
$$

Our final result for $\widehat{\mathcal{J}}_{n(m)}$ is then

$$
\widehat{\mathcal{J}}_{n(m)}=\frac{1}{W} \frac{\prod_{j=0}^{m} \mathcal{J}_{n-m+2 j}}{\prod_{j=1}^{m-1} \chi_{(n-m+2 j)}}\left(\begin{array}{cc}
\Psi_{n+1(m-1)} & -\Psi_{n(m-2)} \\
-\Psi_{n(m)} & \Psi_{n-1(m-1)}
\end{array}\right) .
$$

It remains to prove the claim (E.34). To this end, consider the induced effect of a given row or column transformation of the $\mathcal{J}$-block on the $\mathcal{E}$-block of a matrix factorisation $Q$. According to (A.4), performing e.g. a similarity transformation which adds row $r$ times a polynomial $p$ to row $s$ of the $\mathcal{J}$-block leads to a transformation of the $\mathcal{E}$-block in which the 


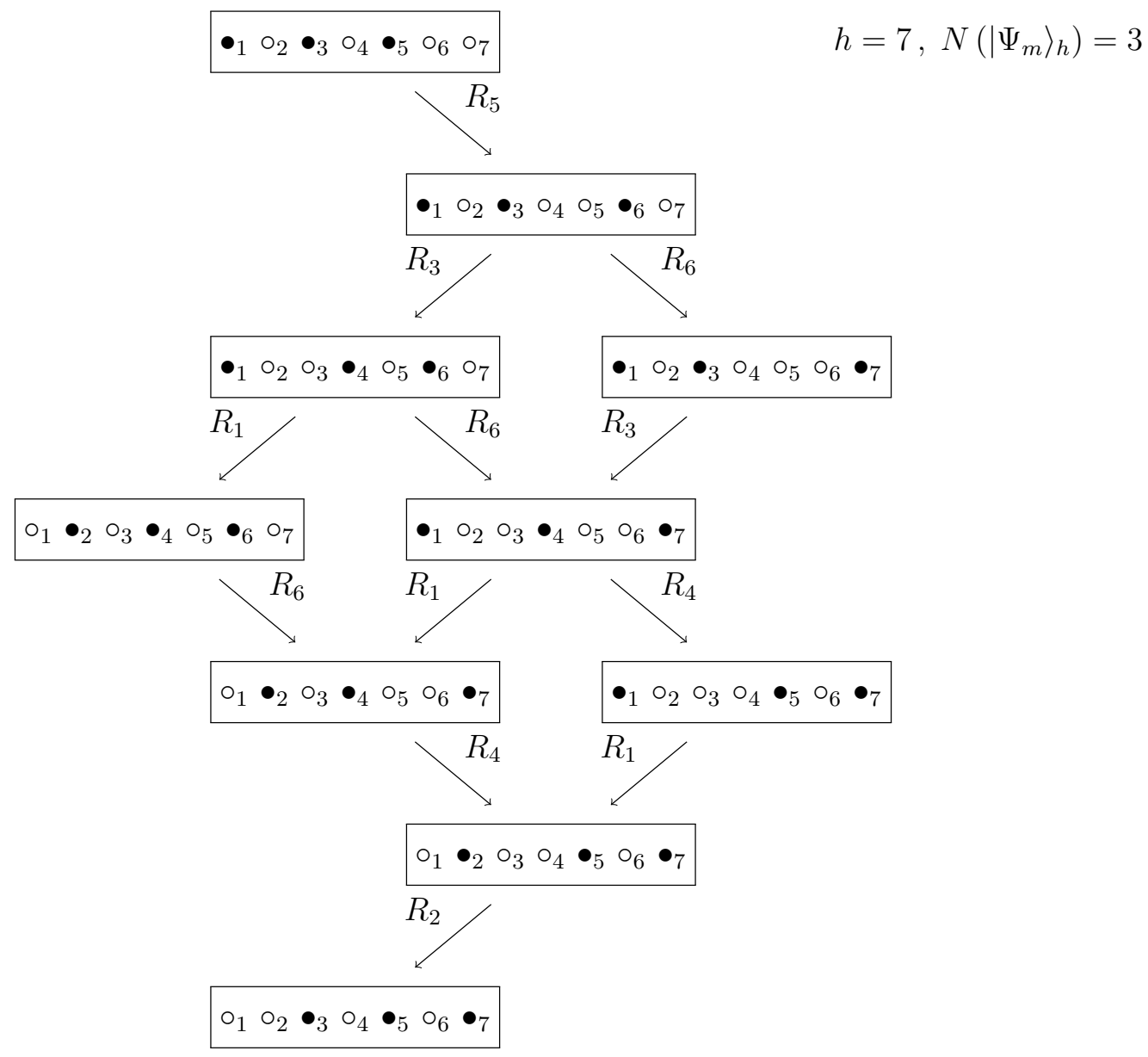

Figure 3. The case $h=7$ and $N=3$.

column $s$ multiplied by $-p$ is added to the column $r$ of the $\mathcal{E}$-block. If we now choose to start constructing the transformation from the form $\mathcal{J}_{n(m)}$ to the form $\widehat{\mathcal{J}}_{n(m)}$ by "clearing out" the entries above the constant entries $\chi_{(p)}$ with a number of row operations, we first of all observe that the subblock of $\mathcal{E}_{n(m)}$ that corresponds to $\widehat{\mathcal{E}}_{n(m)}$ remains unaltered. Similarly, afterwards performing a number of column operations on the $\mathcal{J}_{n(m)}$ block, inducing row transformations on the $\mathcal{E}_{n(m)}$ block, will not affect the $\widehat{\mathcal{E}}_{n(m)}$ subblock. In summary, what we have obtained so far is that one may find a set of similarity transformations that brings the $\mathcal{J}_{n(m)}$ block into the form $\widehat{\mathcal{J}}_{n(m)}$ without affecting the $\widehat{\mathcal{E}}_{n(m)}$ subblock of the $\mathcal{E}_{n(m)}$ block. Now, due to the fact that

$$
\mathcal{J}_{n(m)} \cong \widehat{\mathcal{J}}_{n(m)} \oplus \mathcal{J}_{\text {triv }}^{\oplus m-1}
$$

we automatically must have

$$
\mathcal{E}_{n(m)} \cong \widehat{\mathcal{E}}_{n(m)} \oplus \mathcal{E}_{\text {triv }}^{\oplus m-1},
$$

which concludes the proof. 
Open Access. This article is distributed under the terms of the Creative Commons Attribution License (CC-BY 4.0), which permits any use, distribution and reproduction in any medium, provided the original author(s) and source are credited.

\section{References}

[1] B.R. Greene, String theory on Calabi-Yau manifolds, hep-th/9702155 [INSPIRE].

[2] E. Witten, Phases of $N=2$ theories in two-dimensions, Nucl. Phys. B 403 (1993) 159 [hep-th/9301042] [INSPIRE].

[3] Y. Kazama and H. Suzuki, New $N=2$ superconformal field theories and superstring compactification, Nucl. Phys. B 321 (1989) 232 [INSPIRE].

[4] Y. Kazama and H. Suzuki, Characterization of $N=2$ Superconformal Models Generated by Coset Space Method, Phys. Lett. B 216 (1989) 112 [InSPIRE].

[5] W. Lerche, C. Vafa and N.P. Warner, Chiral Rings in $N=2$ Superconformal Theories, Nucl. Phys. B 324 (1989) 427 [INSPIRE].

[6] D. Gepner, Fusion rings and geometry, Commun. Math. Phys. 141 (1991) 381 [InSPIRE].

[7] I. Brunner, M. Herbst, W. Lerche and B. Scheuner, Landau-Ginzburg realization of open string TFT, JHEP 11 (2006) 043 [hep-th/0305133] [inSPIRE].

[8] A. Kapustin and Y. Li, D-branes in topological minimal models: the Landau-Ginzburg approach, JHEP 07 (2004) 045 [hep-th/0306001] [INSPIRE].

[9] I. Brunner and M.R. Gaberdiel, The matrix factorisations of the D-model, J. Phys. A 38 (2005) 7901 [hep-th/0506208] [INSPIRE].

[10] I. Brunner and M.R. Gaberdiel, Matrix factorisations and permutation branes, JHEP 07 (2005) 012 [hep-th/0503207] [INSPIRE].

[11] H. Enger, A. Recknagel and D. Roggenkamp, Permutation branes and linear matrix factorisations, JHEP 01 (2006) 087 [hep-th/0508053] [INSPIRE].

[12] C.A. Keller and S. Rossi, Boundary states, matrix factorisations and correlation functions for the E-models, JHEP 03 (2007) 038 [hep-th/0610175] [INSPIRE].

[13] N. Behr and S. Fredenhagen, D-branes and matrix factorisations in supersymmetric coset models, JHEP 11 (2010) 136 [arXiv: 1005.2117] [INSPIRE].

[14] N. Behr and S. Fredenhagen, Variable transformation defects, Proc. Symp. Pure Math. 85 (2012) 303 [arXiv:1202.1678] [INSPIRE].

[15] M. Kontsevich, unpublished.

[16] A. Kapustin and Y. Li, D branes in Landau-Ginzburg models and algebraic geometry, JHEP 12 (2003) 005 [hep-th/0210296] [INSPIRE].

[17] D. Orlov, Triangulated categories of singularities and D-branes in Landau-Ginzburg models, math/0302304 [INSPIRE].

[18] A. Kapustin and Y. Li, Topological correlators in Landau-Ginzburg models with boundaries, Adv. Theor. Math. Phys. 7 (2004) 727 [hep-th/0305136] [INSPIRE].

[19] M. Herbst, C.-I. Lazaroiu and W. Lerche, D-brane effective action and tachyon condensation in topological minimal models, JHEP 03 (2005) 078 [hep-th/0405138] [INSPIRE]. 
[20] S. Govindarajan, H. Jockers, W. Lerche and N.P. Warner, Tachyon condensation on the elliptic curve, Nucl. Phys. B 765 (2007) 240 [hep-th/0512208] [INSPIRE].

[21] I. Brunner and D. Roggenkamp, B-type defects in Landau-Ginzburg models, JHEP 08 (2007) 093 [arXiv: 0707.0922] [INSPIRE].

[22] A. Kapustin and L. Rozansky, On the relation between open and closed topological strings, Commun. Math. Phys. 252 (2004) 393 [hep-th/0405232] [INSPIRE].

[23] M. Khovanov and L. Rozansky, Matrix factorizations and link homology, Fund. Math. 199 (2008) 1 [math/0401268].

[24] Y. Yoshino, Tensor products of matrix factorizations, Nagoya Math. J. 152 (1998) 39.

[25] N. Carqueville and I. Runkel, On the monoidal structure of matrix bi-factorisations, J. Phys. A 43 (2010) 275401 [arXiv:0909.4381] [INSPIRE].

[26] J. Fröhlich, J. Fuchs, I. Runkel and C. Schweigert, Duality and defects in rational conformal field theory, Nucl. Phys. B 763 (2007) 354 [hep-th/0607247] [INSPIRE].

[27] D. Gepner, Scalar field theory and string compactification, Nucl. Phys. B 322 (1989) 65 [INSPIRE].

[28] H. Ooguri, Y. Oz and Z. Yin, D-branes on Calabi-Yau spaces and their mirrors, Nucl. Phys. B 477 (1996) 407 [hep-th/9606112] [INSPIRE].

[29] J.L. Cardy, Boundary conditions, fusion rules and the Verlinde formula, Nucl. Phys. B 324 (1989) 581 [INSPIRE].

[30] H. Ishikawa and T. Tani, Twisted boundary states in Kazama-Suzuki models, Nucl. Phys. B 678 (2004) 363 [hep-th/0306227] [INSPIRE].

[31] M.R. Gaberdiel and T. Gannon, Boundary states for WZW models, Nucl. Phys. B 639 (2002) 471 [hep-th/0202067] [INSPIRE].

[32] S. Fredenhagen and V. Schomerus, Brane dynamics in CFT backgrounds, hep-th/0104043 [INSPIRE].

[33] S. Fredenhagen and V. Schomerus, D-branes in coset models, JHEP 02 (2002) 005 [hep-th/0111189] [INSPIRE].

[34] S. Fredenhagen, D-brane dynamics in curved backgrounds, Ph.D. Thesis, Humboldt University, Berlin Germany (2002),

http://edoc.hu-berlin.de/docviews/abstract.php?id=10498.

[35] S. Fredenhagen and V. Schomerus, On boundary RG flows in coset conformal field theories, Phys. Rev. D 67 (2003) 085001 [hep-th/0205011] [InSPIRE].

[36] S. Fredenhagen, Organizing boundary RG flows, Nucl. Phys. B 660 (2003) 436 [hep-th/0301229] [INSPIRE].

[37] C. Bachas and S. Monnier, Defect loops in gauged Wess-Zumino-Witten models, JHEP 02 (2010) 003 [arXiv:0911.1562] [INSPIRE].

[38] V.B. Petkova and J.B. Zuber, Generalized twisted partition functions, Phys. Lett. B 504 (2001) 157 [hep-th/0011021] [INSPIRE].

[39] M. Nozaki, Comments on D-branes in Kazama-Suzuki models and Landau-Ginzburg theories, JHEP 03 (2002) 027 [hep-th/0112221] [INSPIRE]. 
[40] K. Graham and G.M.T. Watts, Defect lines and boundary flows, JHEP 04 (2004) 019 [hep-th/0306167] [INSPIRE].

[41] S.K. Ashok, E. Dell'Aquila and D.-E. Diaconescu, Fractional branes in Landau-Ginzburg orbifolds, Adv. Theor. Math. Phys. 8 (2004) 461 [hep-th/0401135] [InSPIRE].

[42] S.K. Ashok, E. Dell'Aquila, D.-E. Diaconescu and B. Florea, Obstructed D-branes in Landau-Ginzburg orbifolds, Adv. Theor. Math. Phys. 8 (2004) 427 [hep-th/0404167] [INSPIRE].

[43] M. Herbst and C.-I. Lazaroiu, Localization and traces in open-closed topological Landau-Ginzburg models, JHEP 05 (2005) 044 [hep-th/0404184] [INSPIRE].

[44] I. Brunner, N. Carqueville and D. Plencner, Discrete torsion defects, Commun. Math. Phys. 337 (2015) 429 [arXiv:1404.7497] [INSPIRE].

[45] I. Brunner, N. Carqueville and D. Plencner, Orbifolds and topological defects, Commun. Math. Phys. 332 (2014) 669 [arXiv:1307.3141] [INSPIRE].

[46] N. Carqueville and D. Murfet, Adjunctions and defects in Landau-Ginzburg models, arXiv:1208.1481 [INSPIRE].

[47] N. Carqueville and I. Runkel, Rigidity and defect actions in Landau-Ginzburg models, Commun. Math. Phys. 310 (2012) 135 [arXiv:1006.5609] [InSPIRE].

[48] N. Behr, S. Fredenhagen, Rational defects in Landau-Ginzburg models, in preparation.

[49] N. Carqueville and D. Murfet, Computing Khovanov-Rozansky homology and defect fusion, Topology 14 (2014) 489 [arXiv:1108.1081] [INSPIRE].

[50] N. Behr, S. Fredenhagen, Fusion functors, in preparation. 